\title{
National implementation with partially honest agents
}

Citation for published version (APA):

Lombardi, M., \& Yoshihara, N. (2012). National implementation with partially honest agents. METEOR, Maastricht University School of Business and Economics. METEOR Research Memorandum No. 005 https://doi.org/10.26481/umamet.2012005

Document status and date:

Published: 01/01/2012

DOI:

10.26481/umamet.2012005

Document Version:

Publisher's PDF, also known as Version of record

\section{Please check the document version of this publication:}

- A submitted manuscript is the version of the article upon submission and before peer-review. There can be important differences between the submitted version and the official published version of record.

People interested in the research are advised to contact the author for the final version of the publication, or visit the DOI to the publisher's website.

- The final author version and the galley proof are versions of the publication after peer review.

- The final published version features the final layout of the paper including the volume, issue and page numbers.

Link to publication

\footnotetext{
General rights rights.

- You may freely distribute the URL identifying the publication in the public portal. please follow below link for the End User Agreement:

www.umlib.nl/taverne-license

Take down policy

If you believe that this document breaches copyright please contact us at:

repository@maastrichtuniversity.nl

providing details and we will investigate your claim.
}

Copyright and moral rights for the publications made accessible in the public portal are retained by the authors and/or other copyright owners and it is a condition of accessing publications that users recognise and abide by the legal requirements associated with these

- Users may download and print one copy of any publication from the public portal for the purpose of private study or research.

- You may not further distribute the material or use it for any profit-making activity or commercial gain

If the publication is distributed under the terms of Article $25 \mathrm{fa}$ of the Dutch Copyright Act, indicated by the "Taverne" license above, 


\section{Maastricht University}

Michele Lombardi, Naoki Yoshihara

Natural implementation with partially honest agents

$\mathrm{RM} / 12 / 005$

\section{METEOR}

Maastricht University School of Business and Economics

Maastricht Research School of Economics

of Technology and Organization

P. B. Box 616

NL -6200 MD Maastricht

The Netherlands 


\title{
Natural implementation with partially honest agents*
}

\author{
Michele Lombardi $^{\dagger} \quad$ Naoki Yoshihara ${ }^{\ddagger}$ \\ January 31, 2012
}

\begin{abstract}
The paper proposes necessary and sufficient conditions for the natural implementation of (efficient) social choice correspondences $(S C C \mathrm{~s})$ in pure finite exchange economies when some of the agents are partially honest. A partially honest agent is an agent who strictly prefers to tell the truth when lying has no better material consequences for her. Firstly, it is shown that if there is even one partially honest agent in the economy (and the planner does not know her identity), then any $S C C$ is Nash implementable by a natural price-allocation mechanism. Secondly, and in sharp contrast with the results of conventional models of natural implementation, it is shown that the equivalence relationship between natural price-allocation mechanisms and natural price-quantity ${ }^{2}$ mechanisms no longer holds. Finally, and even more strikingly, the paper reports that the class of implementable $S C C$ s by natural price-quantity mechanisms is significantly enlarged.
\end{abstract}

JEL classification: C72; D71.

Key-words: Natural implementation, Nash equilibrium, exchange economies, intrinsic preferences for honesty.

${ }^{*}$ We are grateful to Hans Peters, Tastuyoshi Saijo, Giacomo Valletta, and Dries Vermeulen, for useful comments and suggestions. The first author is also grateful for hospitality received in Japan at the Institute of Economic Research of the Hitotsubashi University, where part of this work was completed. The usual caveat applies.

$\dagger$ Department of Quantitative Economics, Maastricht University, P.O. Box 616, NL-6200 MD Maastricht, Netherlands, phone: 003143388 3761, fax: 003143388 2000, e-mail: m.lombardi@maastrichtuniversity.nl. School of Economics, University of Surrey, GU2 7XH Guildford, Surrey, United Kingdom.

${ }_{\ddagger}^{\ddagger}$ Institute of Economic Research, Hitotsubashi University, 2-4 Naka, Kunitachi, Tokyo, 186-8603 Japan, phone: 008142580 8354, fax: 008142580 8333, e-mail: yosihara@ier.hit-u.ac.jp. 


\section{Introduction}

Nash implementation aims at reaching allocations that satisfy a pre-specified social welfare criterion in situations in which the mechanism designer does not have all necessary relevant information, but rather needs to elicit it from the agents. To this end, the mechanism designer devises a mechanism, which represents a complete description of the set of actions available to each agent and of the consequences of these actions. When only the Nash equilibrium outcomes of the mechanism coincide with the allocations satisfying the given welfare criterion, this welfare criterion is Nash implementable. The allocations that can be implemented as Nash equilibrium in classical exchange economies are now well understood when it is assumed that the agents are only concerned with their own material gains. ${ }^{1}$

This basic tenet is frequently criticized for excluding honesty as a powerful motivator. ${ }^{2}$ In fact, simple reasoning and everyday observation suggest that a concern about honesty is an important determinant of behavior. Furthermore, actual behavior is often the outcome of a compromise between what honesty prescribes and what the pursuit of material gains dictates. Experimental evidence confirms these impressions. As documented in Green et al. (2009), and Hurkens and Kartik (2009), experimental subjects adhere to the civic duty of honesty in the absence of opportunistic behavior. In other words, subjects have an intrinsic motivation toward honesty, but the maximization of their material gains acts as a constraint to this motivation.

This finding is consistent with the conventional views that agents are selfish and selfinterested, and that relations are impersonal in market contexts. This consistency is stressed by Jon Elster who states "Cutthroat competitiveness in the market can go together with strict adherence to norms of honesty" (Elster, 1989; p. 102). Yet, barring the few exceptions discussed below, the implementation literature has neglected the role of honesty as a motivator of human behavior and has failed to appreciate its influence on the design of market-like mechanisms.

This paper follows Elster's perspective and studies the question of which welfare criterion can be implemented in pure finite exchange economies when their participants uphold an intrinsic motivation toward honesty. This study is conducted by focusing on natural mechanisms (Saijo et al., 1996a; 1999). This paper demonstrates that even a 'minimal' propensity toward honesty of the participants enlarges considerably the class of implementable welfare criteria.

This paper assumes complete information among the agents, and that all participants in the economy are Nash competitors. Furthermore, it assumes that in the economy there are partially honest agents. A partially honest participant is an agent who strictly prefers to be truthful in her reports when a lie does not better serve her material interests, given the actions of the other agents. Briefly, the agent at issue can be viewed as having lexicographic preferences for action profiles in which she is concerned first, with material gains, and second, with truthful behavior. The paper also assumes that the mechanism designer knows that there are partially honest agents in the economy but does not know their identities or their exact number.

The role of honesty is quantified by devising only natural mechanisms. ${ }^{3}$ These mecha-

\footnotetext{
${ }^{1}$ Henceforth, by implementation we mean Nash implementation. The seminal paper is Maskin (1999). For recent surveys see, for instance, Jackson (2001) and Maskin and Sjöström (2002).

${ }^{2}$ For instructive discussions on the role of emotions and norms in economics, see Bowles and Gintis (2000), Camerer (2003), Elster (1998), Kreps (1997), Sen (1997), and Suzumura and Xu (2001).

${ }^{3}$ Simple mechanisms introduced by Thomson (2005) are very similar in spirit to natural mechanisms,
} 
nisms have straightforward economic interpretations because agents' actions consist of announcing consumption bundles and prices. Moreover, these mechanisms have several other economically meaningful properties. In fact, following Saijo et al. (1996a; 1999), the paper demands the mechanism (i) to be of finite dimension, (ii) to be individually feasible and balanced, (iii) to satisfy the regularity condition of forthrightness, and (iv) to satisfy the best response property. ${ }^{4}$ An individually feasible and balanced mechanism implies that, in and out of equilibrium, each agent always receives a consumption bundle that lies in her consumption set, and the aggregate consumption is equal to the aggregate social endowments of commodities. The forthrightness property requires that, in equilibrium, each agent obtains what she has reported as her consumption bundle. Finally, the best response property requires each participant to have a best response action for any given actions by the other agents. $^{5}$

Within the class of natural mechanisms, the paper considers four types of mechanisms in which each agent's action consists of reporting (1) a price vector and a consumption bundle, (2) a price vector and two consumption bundles, (3) a price vector and $n-1$ consumption bundles, ${ }^{6}$ and (4) a price vector and an allocation (an entire profile of bundles). These four types of natural mechanisms are termed, respectively, price-quantity, price-quantity ${ }^{2}$, price-quantity $^{n-1}$, and price-allocation mechanisms. The paper limits its analysis to finite pure exchange economies, and identifies the class of efficient welfare criteria - summarized in social choice correspondences $(S C C$ s $)$ - that are implementable by each type of mechanism.

Before discussing the implications and novelty of our findings, it may be worthwhile to emphasize that the departure from the standard implementation set-up is very limited. Like the standard framework, the mechanism designer's implementation problem consists of designing a natural mechanism in such a way that, regardless of what the characteristics of the current economy are and who the partially honest agents involved in the allocation process are, only the $S C C$-optimal allocations emerge as the equilibrium outcomes from the devised mechanism. Moreover, a truly minimal degree of honesty is injected into implementation problems. This is formulated by explicating what constitutes a truthful report in this study. Because a common feature of the four types of natural mechanisms is that an agent's action is made up of two announcement components - one representing prices, and the other representing consumption bundles - this paper assumes that an agent is honest when only the consumption bundles component reported by this agent is consistent with the pre-specified welfare criterion for the underlying economy.

The main results of the paper can be summarized as follows. In the case of natural pricequantity mechanisms, the presence of partially honest agents enlarges in a significant way the class of implementable $S C C$ s with respect to the class that is implementable in the conventional framework. In contrast to the findings of Saijo et al. (1999), this paper reports not only that the constrained Walrasian correspondence is implementable, but also that the (un-

though different in substance. Because our objective is to investigate how the implementability is affected when there are partially-honest agents, rather than studying the implementability of specific welfare criteria, the paper focuses on natural mechanisms.

${ }^{4}$ The pioneers of the approach of setting several desirable properties for a mechanism are Dutta et al. (1995) and Sjöström (1996). See Saijo et al. (1996a; 1999) for a detailed discussion on the implications of the properties listed above. For an instructive study of informational properties of resource-allocation mechanisms, see Hurwicz (1986b).

${ }^{5}$ See Saijo et al. (1996a; 1999), along with the references cited therein, for a detailed discussion on the implications of these properties.

${ }^{6} n \geq 2$ is the number of competing agents in our exchange economies. 
constrained) Walrasian correspondence is implementable in two-commodity economies. The latter finding is even more striking than the former because the Walrasian correspondence is not implementable in the conventional framework.

While the class of implementable $S C C$ s is further enlarged when natural price-quantity ${ }^{2}$ mechanisms are applied, this paper shows a more surprising finding that any efficient $S C C$ is implementable when natural price-quantity ${ }^{n-1}$ mechanisms are applied. This result runs counter to conventional results, which dictate that only monotonic $S C C$ s are implementable. Moreover, while the analysis reveals that implementation by natural price-quantity ${ }^{n-1}$ mechanisms is equivalent to implementation by natural price-allocation mechanisms, the equivalence relationship between implementation by natural price-quantity ${ }^{2}$ mechanisms and implementation by natural price-allocation mechanisms no longer holds. For instance, the efficient and egalitarian-equivalent correspondence is implementable by a natural price-allocation mechanism, but it is not implementable by any natural price-quantity ${ }^{2}$ mechanism. $^{7}$

\subsection{Literature review}

As a final entry to this section, we present the study and its findings within the growing literature on implementation with partially honest agents. ${ }^{8}$ The papers most closely related to this study are those by Dutta and Sen (2012) and Lombardi and Yoshihara (2011a). ${ }^{9}$

Dutta and Sen partially quantified the effect of honesty in abstract social choice environments. We borrow from that paper the concept of partial honesty. Dutta and Sen's main finding is that, when there are three or more agents, any $S C C$ satisfying the condition of no-veto power is implementable. To get this result, the authors focussed on Maskin-type mechanisms, which should be contrasted with the types of natural mechanisms studied in this paper. For instance, in Maskin-type mechanisms each agent's announcement includes either whole preference profiles or whole indifference sets for several agents. In the economic applications on which we focus, these are infinite-dimensional.

Drawing primarily from the above seminal work on the subject, Lombardi and Yoshihara addressed the issue of information decentralization and efficiency in mechanisms. As informationally decentralized mechanisms, the authors take Saijo-type mechanisms (Saijo, 1988; Lombardi and Yoshihara, 2011b), where each agent must announce her own type and that of her neighbor, an alternative, and an integer (henceforth, s-mechanisms). One main finding is that, in contrast to the case of Maskin-type mechanisms, a weaker variant of Maskin monotonicity is necessary to fully identify the class of implementable $S C C$ s by $s$-mechanisms. Consequently, several efficient $S C C \mathrm{~s}$, such as the efficient and egalitarianequivalent correspondence and the Walrasian correspondence, are not implementable via

\footnotetext{
${ }^{7}$ Similar results, though they differ in substance from those reported here, are found in Lombardi and Yoshihara (2011c) when only quantity announcements are allowed.

${ }^{8}$ The impressive body of evidence accumulated by psychologists over the past two decades has caused scholars to study the implications of weakening other fundamental assumptions in a variety of ways and has already produced a number of alternatives to the standard implementation model, for instance, Eliaz, 2002; Glazer and Rubinstein, 1998; Cabrales and Serrano, 2011. Notably, the first paper on 'behavioral implementation theory' dates from 1986, in which Hurwicz solves the implementation problem without positing the completeness nor the transitivity of agents' preferences (Hurwicz, 1986a).

${ }^{9}$ Kartik and Tercieux (2011) enrich the standard implementation framework by allowing agents to report evidence. In these environments, they identify a necessary condition for implementability, called evidencemonotonicity. This condition, when combined with no-veto power, is sufficient for implementation with evidence. In a society with partially-honest agents, every $S C C$ is evidence-monotonic because of DuttaSen's definition per se of partially-honest agents' orderings over message profiles. Consequently, Kartik and Tercieux's result is similar to Dutta and Sen's result, summarized above.
} 
this type of mechanism. It is intriguing, however, that those $S C C$ s are implementable when more demanding mechanisms such as natural price-allocation mechanisms are applied. Note that natural price-allocation mechanisms are much more informationally efficient than $s$-mechanisms both in quantitative as well as qualitative senses. First, quantitatively speaking, the strategy space of each participant in natural price-allocation mechanisms is much 'smaller' than that in $s$-mechanisms, in the sense that the former is finite-dimensional whereas the latter is infinite-dimensional. Second, qualitatively speaking, all components of each message in price-allocation mechanisms consist solely of self-relevant information, while they do not in $s$-mechanisms. ${ }^{10}$ This noticeable difference makes our result of natural price-allocation mechanisms more compelling.

Other related papers are by Corchón and Herrero (2004) and Matsushima (2008). Matsushima is the first author who quantified the role of honesty in implementation theory. Matsushima shows that if a social choice function is Bayesian-incentive compatible and agents have the standard, quasi-linear preferences over outcomes and suffer a small utility loss from lying, then this social choice function is implementable in iteratively undominated strategies. Therefore, it is also implementable in Bayesian Nash equilibrium when there are more than two participants. Clearly, the main body of this paper is substantially different from Matsushima (2008). First, in contrast to Matsushima (2008), this paper does not need to presume that all agents should have intrinsic preferences for honesty but needs only to presume that at least one agent has such preferences. Second, this paper focuses on implementation problems with complete information, while Matsushima (2008) concentrated on problems with incomplete information.

Corchón and Herrero introduce decency requirements for the set of admissible messages that depend on the true preferences of the outcomes of agents, and investigate their effects on the class of implementable $S C C$ s. Given a particular formulation of these requirements, these authors show that a stronger variant of no-veto power is sufficient for implementation in decent strategies. In contrast, imposing no condition on the set of messages conveyed by an agent, this paper instead assumes that each agent has a complete preference order of message profiles, which is determined as much by her intrinsic taste for the bundle brought about by each profile as by her sense of honesty.

The remainder of the paper is structured as follows. Section 2 describes a formal model. Section 3 reports the analysis for price-quantity ${ }^{n-1}$ and price-allocation mechanisms. Sections 4 and 5 cover price-quantity ${ }^{2}$ and price-quantity mechanisms, respectively. Section 6 discusses briefly the implications of the results reported in sections 4 and 5 . Section 7 reports the analysis for two-agent economies. Section 8 concludes briefly. Long proofs are collected in the Appendix.

\section{The Model}

There are $n \geq 2$ agents or participants in $N \equiv\{1, \ldots, n\}$ and $\ell \geq 2$ distinct commodities in $L \equiv\{1, \ldots, \ell\}$. Unless otherwise specified, we assume that the cardinality of $L$ is $\ell \geq$ 2. $\mathbb{R}$ is the set of all real numbers; $\mathbb{R}_{+}$(resp., $\mathbb{R}_{++}$) denotes the set of all non-negative

\footnotetext{
${ }^{10}$ Indeed, natural price-allocation mechanisms can be regarded as examples of self-relevant mechanisms (Hurwicz, 1960), as Tatamitani (2001) pointed out. In a recent paper, Lombardi and Yoshihara (2011d) investigate implementation problems by self-relevant mechanisms when some of the participants are partially honest.
} 
(respectively, positive) real numbers; $\mathbb{R}^{\ell}$ is the Cartesian product of ordered $\ell$-tuples of real numbers, whereas $\mathbb{R}_{+}^{\ell}$ (resp., $\mathbb{R}_{++}^{\ell}$ ) denotes its non-negative (resp., positive) orthant. Vector inequalities are defined as follows: for all $x, y \in \mathbb{R}^{\ell}, x \geq y$ if $x_{\ell} \geq y_{\ell}$ for each $\ell \in L, x>y$ if $x \geq y$ and $x \neq y$, and $x \gg y$ if $x_{\ell}>y_{\ell}$ for each $\ell \in L$.

Each agent $i \in N$ is characterized by a consumption space $\mathbb{R}_{+}^{\ell}\left(\right.$ where $x_{i}=\left(x_{i 1}, \ldots, x_{i \ell}\right) \in$ $\mathbb{R}_{+}^{\ell}$ is the agent $i$ 's commodity bundle), by an endowment vector $\omega_{i} \in \mathbb{R}_{+}^{\ell}$, and by a preference relation defined over $\mathbb{R}_{+}^{\ell}$. We assume that agent $i$ 's preferences have a utility representation $u_{i}: \mathbb{R}_{+}^{\ell} \rightarrow \mathbb{R}$ which is continuous and quasi-concave on $\mathbb{R}_{+}^{\ell}$, and either strictly monotonic on $\mathbb{R}_{+}^{\ell}$ or strictly monotonic on $\mathbb{R}_{++}^{\ell}$ where the utility of every interior consumption bundle is strictly higher than the utility of any consumption bundle on the boundary. $U$ is the class of all such utility functions, whereas $U_{i}$ is the class of admissible utility functions for agent $i \in N$. Given a profile of endowment vectors, we denote $\sum_{i \in N} \omega_{i} \equiv \Omega \in \mathbb{R}_{++}^{\ell}$ as the aggregate endowment. It is assumed that the distribution of endowments is known and fixed. For $\left(i, u_{i}, x_{i}\right) \in N \times U_{i} \times \mathbb{R}_{+}^{\ell}, L\left(x_{i}, u_{i}\right) \equiv\left\{x_{i}^{\prime} \in \mathbb{R}_{+}^{\ell} \mid x_{i}^{\prime} \leq \Omega\right.$ and $\left.u_{i}\left(x_{i}\right) \geq u_{i}\left(x_{i}^{\prime}\right)\right\}$ denotes the weak lower contour set for agent $i$ with $u_{i}$ at $x_{i}$, whereas $\partial L\left(x_{i}, u_{i}\right)$ denotes the upper boundary of $L\left(x_{i}, u_{i}\right)$, that is, $\partial L\left(x_{i}, u_{i}\right) \equiv\left\{x_{i}^{\prime} \in L\left(x_{i}, u_{i}\right) \mid u_{i}\left(x_{i}\right)=u_{i}\left(x_{i}^{\prime}\right)\right\}$. An economy or state is specified by a list $u=\left(u_{i}\right)_{i \in N} \in U_{N} \equiv \times_{i \in N} U_{i}$. An allocation is a list of bundles $x=\left(x_{1}, \ldots, x_{n}\right) \in \mathbb{R}_{+}^{n \ell}$, whereas a feasible allocation is an allocation $x=\left(x_{1}, \ldots, x_{n}\right) \in \mathbb{R}_{+}^{n \ell}$ such that $\sum_{i \in N} x_{i}=\Omega$. The set of all feasible allocations is denoted by $A$. For any allocation $x \in \mathbb{R}_{+}^{n \ell}$ and any (non-empty) set of agents $\varnothing \neq T \subset N, x_{-T} \equiv\left(x_{i}\right)_{i \in N \backslash T}$ is the list of bundles for the agents in $N \backslash T .{ }^{11}$ Given $x_{T} \in \mathbb{R}_{+}^{t \ell}$ and $x_{-T} \in \mathbb{R}_{+}^{(n-t) \ell}$, we denote $\left(x_{T}, x_{-T}\right)$ as the allocation consisting of these $x_{T}$ and $x_{-T}$. Given a set $S \subset \mathbb{R}_{+}^{n}$, its boundary and interior are denoted $\partial S$ and $\stackrel{\circ}{S}$, respectively.

A social choice correspondence $(S C C)$ is a multi-valued mapping $F: U_{N} \rightarrow A$ such that for each $u \in U_{N}, F(u)$ is a non-empty subset of feasible allocations, that is, $\varnothing \neq F(u) \subseteq A$. Unless specified otherwise, we do not assume that for all $u \in U_{N}$ and all $x \in F(u), x_{i} \gg 0$ for all $i \in N$. The set of (Pareto) efficient allocations for the economy $u \in U_{N}$, denoted $P(u)$, is $P(u) \equiv\left\{x \in A \mid\right.$ there is no $y \in A: u_{i}\left(y_{i}\right)>u_{i}\left(x_{i}\right)$ for all $\left.i \in N\right\}$. An $S C C F$ defined on $U_{N}$ is efficient if for any $u \in U_{N}, F(u) \subseteq P(u)$ holds. $\mathcal{F}$ is the class of all efficient $S C C$ s defined on $U_{N}$.

For any $\left(u_{i}, x_{i}\right) \in U_{i} \times \mathbb{R}_{+}^{\ell}, V_{i}\left(x_{i}, u_{i}\right) \equiv\left\{y_{i} \in \mathbb{R}_{+}^{\ell} \mid y_{i} \leq \Omega\right.$ and $\left.u_{i}\left(x_{i}\right) \leq u_{i}\left(y_{i}\right)\right\}$ denotes the weak upper contour set for agent $i$ with $u_{i}$ at $x_{i}$. Given $\left(u_{i}, x_{i}\right) \in U_{i} \times \mathbb{R}_{+}^{\ell}$, a price vector $p$ belonging to the unit simplex $\Delta$, that is, $p \in \Delta$, is said to be a sub-gradient of $u_{i}$ at $x_{i}$ if $p \cdot x_{i}^{\prime} \geq p \cdot x_{i}$ for all $x_{i}^{\prime} \in V_{i}\left(x_{i}, u_{i}\right)$. The set of all sub-gradients of $u_{i}$ at $x_{i}$, that is, $\delta u_{i}\left(x_{i}\right) \equiv\left\{p \in \Delta \mid p \cdot x_{i}^{\prime} \geq p \cdot x_{i}\right.$ for all $\left.x_{i}^{\prime} \in V_{i}\left(x_{i}, u_{i}\right)\right\}$, is called the sub-differential of $u_{i}$ at $x_{i}$. For any $(x, u) \in \mathbb{R}_{+}^{n \ell} \times U_{N}$, let $\Pi(x, u) \equiv \cap_{i \in N} \delta u_{i}\left(x_{i}\right)$. Notice that $x \in P(u)$ if $\Pi(x, u)$ is non-empty. In words, $\Pi(x, u)$ consists of prices $p$ each of which is normal to a hyperplane separating the weak upper contour sets of all agents with $u$ at $x$. Any $p \in \Pi(x, u)$ is referred to as a Pareto efficient price for $u$ at $x$. If $u_{i} \in U_{i}$ is differentiable for all $i \in N$ and $F \in \mathcal{F}$ selects only interior allocations, $\varnothing \neq F(u) \subseteq A \cap \mathbb{R}_{++}^{n \ell}$ for any $u \in U_{N}$, then the set $\Pi(x, u)$ is a singleton whenever $x \in F(u)$; in particular, the set $\Pi(x, u)$ has the form of $\{p\} \subseteq \Delta$ such that $D u_{i}\left(x_{i}\right)=p$ for all $i \in N .{ }^{12}$

\footnotetext{
${ }^{11}$ The weak set inclusion is denoted by $\subseteq$, while the strict set inclusion is denoted by $\subset$. The notation " $t$ " means the cardinality of the set $T$.

${ }^{12}$ For $u_{i} \in U_{i}, D u_{i}\left(x_{i}\right)$ denoted the gradient vector at $x_{i}$ which is normalized to belong to the unit simplex
} $\Delta$. 
A mechanism is a pair $\gamma \equiv(M, g)$, where $M \equiv M_{1} \times \ldots \times M_{n}$, with each $M_{i}$ being a (nonempty) set, and $g: M \rightarrow \mathbb{R}^{n \ell}$. It consists of a message space $M$, where $M_{i}$ is the message space for agent $i \in N$, and an outcome function $g$ such that $g(m)=\left(g_{i}(m)\right)_{i \in N} \in \mathbb{R}^{n \ell}$ for each $m \in M . m_{i} \in M_{i}$ denotes a generic message (or strategy) for agent $i$. A message profile is denoted by $m \equiv\left(m_{1}, \ldots, m_{n}\right) \in M$. For any $m \in M$ and $j \in N$, let $m_{-j} \equiv$ $\left(m_{1}, \ldots, m_{j-1}, m_{j+1}, \ldots, m_{n}\right) \in \times_{i \in N \backslash\{j\}} M_{i} \equiv M_{-j}$. Given an $m_{-j} \in M_{-j}$ and an $m_{j} \in M_{j}$, we denote $\left(m_{j}, m_{-j}\right)$ to be the message profile consisting of $m_{j}$ and $m_{-j} . g\left(M_{i}, m_{-i}\right)$ is the attainable set of agent $i$ at $m_{-i}$, that is, the set of bundles that agent $i$ can induce when the other agents select $m_{-i}$. A mechanism is: (i) individually feasible if $g(m) \in \mathbb{R}_{+}^{n \ell}$ for each $m \in M$; (ii) balanced if $\sum_{i \in N} g_{i}(m)=\Omega$ for each $m \in M$; and, (iii) weakly balanced if $\sum_{i \in N} g_{i}(m) \leq \Omega$ for each $m \in M$. An individually feasible and balanced mechanism has the property that $g(m) \in A$ for each $m \in M$. A mechanism satisfies the best response property if, for all $\left(i, u_{i}, m_{-i}\right) \in N \times U_{i} \times M_{-i}$, there exists an $m_{i} \in M_{i}$ such that $u_{i}\left(g_{i}\left(m_{i}, m_{-i}\right)\right) \geq$ $u_{i}\left(g_{i}\left(m_{i}^{\prime}, m_{-i}\right)\right)$ for all $m_{i}^{\prime} \in M_{i}$.

A mechanism $\gamma$ induces a class of (non-cooperative) games $\left\{(\gamma, u) \mid u \in U_{N}\right\}$. Given a game $(\gamma, u)$, we say that $m^{*} \in M$ is a (pure strategy) Nash equilibrium at $u$ if and only if, for all $i \in N, g\left(M_{i}, m_{-i}^{*}\right) \subseteq L\left(g_{i}\left(m^{*}\right), u_{i}\right)$. For any game $(\gamma, u), N E(\gamma, u)$ denotes the set of Nash equilibrium message profiles of $(\gamma, u)$, whereas $N A(\gamma, u)$ represents the corresponding set of Nash equilibrium allocations. A mechanism $\gamma$ implements $F \in \mathcal{F}$ in Nash equilibria, or simply implements $F$, if and only if $F(u)=N A(\gamma, u)$ for all $u \in U_{N}$. If such a mechanism exists, then $F$ is (Nash) implementable.

For any mechanism $\gamma$ and any agent $i \in N$, a truth-telling correspondence is a correspondence $T_{i}^{\gamma}: U_{N} \times \mathcal{F} \rightarrow M_{i}$ such that, for each $(u, F) \in U_{N} \times \mathcal{F}, \varnothing \neq T_{i}^{\gamma}(u, F) \subseteq M_{i}$. An interpretation of the set $T_{i}^{\gamma}(u, F)$ is that, given the mechanism $\gamma$ and a pair $(u, F) \in U_{N} \times \mathcal{F}$, agent $i$ behaves truthfully at the message profile $m \in M$ if and only if $m_{i} \in T_{i}^{\gamma}(u, F)$. In other words, $T_{i}^{\gamma}(u, F)$ is the set of truthful messages of agent $i$ under the mechanism $\gamma$, when the current economy is $u \in U_{N}$ and the social goal is given by $F$. Note that the type of elements of $M_{i}$ constituting $T_{i}^{\gamma}(u, F)$ depends on the type of mechanism $\gamma$ that one may consider. For example, if the message conveyed by each agent to the mechanism designer involves the announcement of an allocation and the relevant notion of truthfulness consists of sending an allocation consistent with the welfare criterion $F \in \mathcal{F}$ for any economy $u \in U_{N}$, then $M_{i}$ may be defined by $M_{i} \equiv M_{i}^{1} \times M_{i}^{2}$, where $T_{i}^{\gamma}(u, F)=\{F(u)\} \times M_{i}^{2}$ for each $(u, F) \in U_{N} \times \mathcal{F}$.

For any $(i, u) \in N \times U$, let $\succcurlyeq_{i}^{u}$ be agent $i$ 's weak order over $M$ under the economy $u$. The asymmetric factor of $\succcurlyeq_{i}^{u}$ is denoted by $\succ_{i}^{u}$, while the symmetric part is denoted by $\sim_{i}^{u}$. For any $u \in U_{N}, \succcurlyeq^{u}$ is the profile of weak orders over $M$ under the economy $u$; in other words, $\succcurlyeq^{u} \equiv\left(\succcurlyeq_{i}^{u}\right)_{i \in N^{*}}$. As in Dutta and Sen (2011), partially honest behavior is defined as follows.

Definition 1. An agent $h \in N$ is a partially honest agent if for any mechanism $\gamma$, any $u \in U_{N}$, any $F \in \mathcal{F}$, any $m \equiv\left(m_{h}, m_{-h}\right)$, and any $m^{\prime} \equiv\left(m_{h}^{\prime}, m_{-h}\right) \in M$, the following properties hold:

(i) if $m_{h} \in T_{h}^{\gamma}(u, F), m_{h}^{\prime} \notin T_{h}^{\gamma}(u, F)$, and $u_{h}\left(g_{h}(m)\right) \geq u_{h}\left(g_{h}\left(m^{\prime}\right)\right)$, then $\left(m, m^{\prime}\right) \in \succ_{h}^{u}$;

(ii) otherwise, $\left(m, m^{\prime}\right) \in \succcurlyeq_{h}^{u}$ if and only if $u_{h}\left(g_{h}(m)\right) \geq u_{h}\left(g_{h}\left(m^{\prime}\right)\right)$.

An agent $i \in N$ who is also a partially honest agent is denoted by $h$. If agent $i \in N$ is not a partially honest agent (that is, $i \neq h$ ), then for each game $(\gamma, u)$ and all $m, m^{\prime} \in M$ : $\left(m, m^{\prime}\right) \in \succcurlyeq_{i}^{u}$ if and only if $u_{i}\left(g_{i}(m)\right) \geq u_{i}\left(g_{i}\left(m^{\prime}\right)\right)$.

Unless otherwise specified, the following informational assumption holds throughout the 
paper.

Assumption 1. There are partially honest agents in $N$. The mechanism designer is well aware of the fact that there are partially honest agents in $N$ but she does not know their identities or their exact number.

Thus, while the mechanism designer knows that there are partially honest agents in the society and how these agents behave, the mechanism designer knows neither the identity of the partially honest agents nor their exact number.

Let $\varnothing \neq \mathcal{H} \subseteq 2^{N} \backslash \varnothing$ be a class of non-empty subsets of $N$. The family $\mathcal{H}$ is viewed as the class of conceivable sets of partially honest agents. That is, if $H \in \mathcal{H}$, then this $H$ is a conceivable set of partially honest agents. By Assumption 1, the mechanism designer knows that $\mathcal{H}$ is non-empty, and she may even know the composition of $\mathcal{H}$, but she never knows what element of $\mathcal{H}$ is the current set of partially honest agents in society. Assumption 1 implies that $\# \mathcal{H} \geq 2$.

A mechanism $\gamma$ induces a class of (non-cooperative) games with partially honest agents $\left\{\left(\gamma, \succcurlyeq^{u}\right) \mid(u, H) \in U_{N} \times \mathcal{H}\right\}$. Given a game $\left(\gamma, \succcurlyeq^{u}\right)$, we say that $m^{*} \in M$ is a (pure strategy) Nash equilibrium with partially honest agents at $u$ if and only if, for all $i \in N$, $\left(m^{*},\left(m_{i}, m_{-i}^{*}\right)\right) \in \succcurlyeq_{i}^{u}$ for all $m_{i} \in M_{i}$. Given a game $\left(\gamma, \succcurlyeq^{u}\right), N E\left(\gamma, \succcurlyeq^{u}\right)$ denotes the set of Nash equilibrium message profiles of $\left(\gamma, \succcurlyeq^{u}\right)$, whereas $N A\left(\gamma, \succcurlyeq^{u}\right)$ represents the corresponding set of Nash equilibrium allocations.

Definition 2. A mechanism $\gamma$ partially honestly implements $F \in \mathcal{F}$ in Nash equilibria, or simply partially honestly implements $F$, if and only if $F(u)=N A\left(\gamma, \succcurlyeq^{u}\right)$ for all $u \in U_{N}$ and all $H \in \mathcal{H}$.

If such a mechanism exists, then $F$ is partially honest (Nash) implementable.

Note that this definition of implementation is similar, but not identical, to the standard definition. ${ }^{13}$ First, the Nash equilibrium allocations are given by the game $\left(\gamma, \succcurlyeq^{u}\right)$ rather than by the game $(\gamma, u)$. Second, the equivalence of the set of $S C C$-optimal allocations with the set of Nash equilibrium allocations is required not only for any economy $u \in U_{N}$, but also for any conceivable set of partially honest agents $H \in \mathcal{H}$. The latter part of the definition presented above captures the distinctiveness of our implementation models. Indeed, in these models, a preference over message profiles for each participant does not necessarily coincide with her preference over allocations, and the mechanism designer has no information of these preferences. ${ }^{14}$

\section{Price-quantity ${ }^{n-1}$ and price-allocation mechanisms}

This section explores the natural implementation of $S C C$ s via natural price-allocation mechanisms and price-quantity ${ }^{n-1}$ mechanisms.

While in a price-allocation mechanism each participant states a price vector and an allocation, a price-quantity ${ }^{n-1}$ mechanism is a game form in which each agent reports to the mechanism designer a price vector, her consumption bundle, and the consumption bundles of the other $n-2$ participants. A way to proceed is to arrange agents clockwise facing inward,

\footnotetext{
${ }^{13}$ However, the two definitions are identical if $\mathcal{H}=\varnothing$.

${ }^{14}$ In the standard set-up, preferences of allocations are equivalent to preferences of message profiles for each participant.
} 
and require that each participant $i$ announces, inter alia, her consumption bundle and the consumption bundles of the $n-2$ participants standing to her left. ${ }^{15}$

Requiring forthrightness, individual feasibility, balancedness, and the best response property, partially honest implementation by natural price-allocation mechanisms and by natural price-quantity $^{n-1}$ mechanisms can be defined as follows.

Definition 3. An $S C C F \in \mathcal{F}$ is partially honest implementable by a natural priceallocation mechanism if there exists a mechanism $\gamma$ such that:

(i) $\gamma$ partially honestly implements $F$;

(ii) for each $i \in N, M_{i}=\Delta \times A$;

(iii) for each $u \in U_{N}$, each $x \in F(u)$, and each $p \in \Pi(x, u)$, if $m_{i}=(p, x)$ for each $i \in N$, then $m \in N E\left(\gamma, \succcurlyeq^{u}\right)$ and $g(m)=x$;

(iv) $\gamma$ is individually feasible and balanced;

(v) $\gamma$ satisfies the best response property.

Definition 4. An $S C C F \in \mathcal{F}$ is partially honest implementable by a natural pricequantity $^{n-1}$ mechanism if there exists a mechanism $\gamma$ such that:

(i) $\gamma$ partially honestly implements $F$;

(ii) for each $i \in N, M_{i}=\Delta \times Q^{n-1}$, where

$$
Q^{n-1} \equiv\left\{\left(x_{i}, x_{i+1}, \ldots, x_{i+n-2}\right) \in \mathbb{R}_{+}^{(n-1) \ell} \mid \sum_{l=i}^{i+n-2} x_{l} \leq \Omega\right\}
$$

where $i+k$ for $k \in\{1, \ldots, n-2\}$ is regarded as $\ell \in N$ if $i+k=n+\ell>n$;

(iii) for each $u \in U_{N}$, each $x \in F(u)$, and each $p \in \Pi(x, u)$, if $m_{i}=\left(p, x_{i}, x_{i+1}, \ldots, x_{i+n-2}\right)$ for each $i \in N$, then $m \in N E\left(\gamma, \succcurlyeq^{u}\right)$ and $g(m)=x$;

(iv) $\gamma$ is individually feasible and balanced;

(v) $\gamma$ satisfies the best response property.

Condition (iii) of both Definition 3 and Definition 4 is the regularity condition of forthrightness. ${ }^{16}$

We are now ready to define the notion of truthfulness employed in this section for the partially honest participants involved in each of the natural mechanisms just defined. By employing the idea of Dutta and Sen (2012) and Lombardi and Yoshihara (2011a), an agent's message is truthful if it is truthful in its consumption bundles component. Formally, for any economy $u \in U_{N}$, any welfare criterion $F \in \mathcal{F}$, and any agent $i \in N$, the set of truthful messages for a natural price-allocation mechanism $\gamma$ is

$$
T_{i}^{\gamma}(u, F) \equiv\{(p, x) \in \Delta \times A \mid x \in F(u)\},
$$

whereas for a natural price-quantity ${ }^{n-1}$ mechanism $\gamma$, it is

$$
T_{i}^{\gamma}(u, F) \equiv\left\{\left(p, x_{i}, \ldots, x_{i+n-2}\right) \in \Delta \times Q^{n-1} \mid \exists x_{i+n-1}:\left(x_{i}, \ldots, x_{i+n-2}, x_{i+n-1}\right) \in F(u)\right\} .
$$

A key property of the notion of truthful messages is that it is functionally independent of prices announced by the participants. In this sense, our characterization results are

\footnotetext{
${ }^{15}$ This way to proceed is without loss of generality. The following results hold for a more general case in which for each participant there are $n-1$ participants who make announcements of her consumption bundle. A similar way to proceed is employed below for the case of natural price-quantity ${ }^{2}$ mechansims.

${ }^{16}$ Forthrightness is called truthful implementation by Dutta et al. (1995).
} 
provided by injecting only a slight preference for honesty into the natural implementation theory. Nonetheless, the conclusion that can be drawn from the following theorem (whose proof is relegated to the Appendix) is that even this small departure from the conventional framework widens the scope of implementation by natural price-quantity ${ }^{n-1}$ mechanisms dramatically.

Theorem 1. Let $n \geq 3$ and suppose that Assumption 1 holds. Then, every SCC $F \in \mathcal{F}$ is partially honest implementable by a natural price-quantity ${ }^{n-1}$ mechanism.

Saijo et al. (1999) show that the constrained Walrasian correspondence cannot be implemented by any natural mechanism if the common marginal rate of substitution is not uniquely determined and there are more than two commodities, that is, $\ell \geq 3$. A remarkable implication of Theorem 1 is that the (unconstrained) Walrasian correspondence is partially honest implementable by a natural price-quantity ${ }^{n-1}$ mechanism, even though the common marginal rate of substitution is not unique and the equilibrium allocations are not necessarily interior allocations.

It is important to note that for an individually feasible and balanced mechanism, announcing a price vector and $n-1$ consumption bundles is equivalent to announcing a price vector and an allocation. This is because any $n-1$ consumption bundles $\left(x_{i}, \ldots, x_{i+n-2}\right) \in Q^{n-1}$ induce a unique allocation $\left(x_{i}, \ldots, x_{i+n-2}, x_{i+n-1}\right) \in A$, where $x_{i+n-1}=\max \left\{\mathbf{0}, \Omega-\sum_{k=0}^{n-2} x_{i+k}\right\}$. The following result states an obvious implication of this equivalence relationship in the light of Theorem 1.

Corollary 1. Let $n \geq 3$ and suppose that Assumption 1 holds. An SCC $F \in \mathcal{F}$ is partially honest implementable by a natural price-allocation if and only if $F$ is partially honest implementable by a natural price-quantity ${ }^{n-1}$ mechanism.

As a consequence of Theorem 1 and Corollary 1, every efficient $S C C$ is partially honest implementable by a natural price-allocation mechanism.

In classical economic environments, while any efficient $S C C$ is partially honest implementable when no restriction is imposed on the class of available mechanisms, Lombardi and Yoshihara (2011a) showed that several efficient SCCs, such as the efficient and egalitarianequivalent correspondence and the Walrasian correspondence, are not partially honest implementable when only $s$-mechanisms can be employed. Compared to this result, Theorem 1 and Corollary 1 give us considerably more interesting results, because as mentioned in the introduction, a natural price-allocation mechanism incorporates a strategy space reduction which is more significant than that of an $s$-mechanism, regarding the price announcement as part of the self-relevant information.

Before closing this section, it may be worth noting that in the light of Theorem 1 and the equivalence relationship between natural price-allocation mechanisms and natural price quantity $^{n-1}$ mechanisms, all efficient $S C C$ s are partially honest implementable by a natural price-quantity $^{2}$ mechanism in three-agent economies.

Corollary 2. Let $n=3$ and suppose that Assumption 1 holds. Every SCC $F \in \mathcal{F}$ is partially honest implementable by a natural price-quantity ${ }^{2}$ mechanism.

\section{$4 \quad$ Price-quantity ${ }^{2}$ mechanisms}

As shown in Corollary 2, any efficient $S C C$ is partially honest implementable by a natural price-quantity ${ }^{2}$ mechanism in three-agent economies. In other words, the equivalence be- 
tween partially honest implementation by natural price-quantity ${ }^{2}$ mechanisms and partially honest implementation by natural price-allocation mechanisms holds in economies with only three agents, as in the case of the standard framework of natural implementation (see Saijo et al., 1996a; 1999). It is not apparent, however, whether such an equivalence holds when there are four agents or more. This section investigates whether this equivalence relationship continues to hold when there are more than three participants.

To this end, additional notation is needed. For each given $u \in U_{N}$ and $x \in F(u)$, let

$$
F^{-1}(x, u) \equiv\left\{u^{\prime} \in U_{N} \mid x \in F\left(u^{\prime}\right)\right\}
$$

and $\Lambda_{i}^{F}(x, u) \equiv \cap_{u^{\prime} \in F^{-1}(x, u)} L\left(x_{i}, u_{i}^{\prime}\right)$. Moreover, for each $u \in U_{N}$, each $x \in F(u)$, and each $p \in \Pi(x, u)$, define the set $F^{-1}(x, p)$ as

$$
F^{-1}(x, p) \equiv\left\{u^{\prime} \in U_{N} \mid x \in F\left(u^{\prime}\right) \text { and } p \in \Pi\left(x, u^{\prime}\right)\right\}
$$

whereas the sets $F_{!}^{-1}(x, p)$ and $\Pi^{F}(x, u)$ are defined respectively as follows:

$$
\begin{gathered}
F_{!}^{-1}(x, p) \equiv\left\{u^{\prime} \in U_{N} \mid x \in F\left(u^{\prime}\right) \text { and }\{p\}=\Pi\left(x, u^{\prime}\right)\right\}, \\
\Pi^{F}(x, u) \equiv\left\{p \in \Pi(x, u) \mid F_{!}^{-1}(x, p) \neq \varnothing\right\} .
\end{gathered}
$$

Then, for each $u \in U_{N}$ and each $x \in F(u)$, let us define the set $\pi^{F}(x, u)$ as follows:

$$
\pi^{F}(x, u) \equiv \begin{cases}\Pi^{F}(x, u) & \text { if it is non-empty } \\ \Pi(x, u) & \text { otherwise. }\end{cases}
$$

Finally, for each $u \in U_{N}$, each $x \in F(u)$, and each $p \in \pi^{F}(x, u)$, let $\Lambda_{i}^{F}(x, p) \equiv \cap_{u^{\prime} \in F^{-1}(x, p)} L\left(x_{i}, u_{i}^{\prime}\right)$.

Requiring forthrightness, individual feasibility, balancedness, and the best response property, we define partially honest implementation by a natural price-quantity ${ }^{2}$ mechanism as follows.

Definition 5. An $S C C F \in \mathcal{F}$ is partially honest implementable by a natural price-quantity ${ }^{2}$ mechanism if there exists a mechanism $\gamma$ such that:

(i) $\gamma$ partially honestly implements $F$;

(ii) for each $i \in N, M_{i}=\Delta \times Q^{2}$, where $Q^{2} \equiv\left\{\left(x_{i}, x_{i+1}\right) \in \mathbb{R}_{+}^{2 \ell} \mid x_{i}+x_{i+1} \leq \Omega\right\}$ and $n+1=1$;

(iii) for each $u \in U_{N}$, each $x \in F(u)$, and each $p \in \pi^{F}(x, u)$, if $m_{i}=\left(p, x_{i}, x_{i+1}\right)$ for each $i \in N$, then $m \in N E\left(\gamma, \succcurlyeq^{u}\right)$ and $g(m)=x$, where $n+1=1$;

(iv) $\gamma$ is individually feasible and balanced;

(v) $\gamma$ satisfies the best response property.

Condition (iii) in Definition 5 represents the regularity condition of forthrightness by natural price-quantity $^{2}$ mechanisms and differs slightly from that imposed by Saijo et al. (1999; Definition 3(iii)). The difference between the two conditions is that Saijo et al. (1999)'s condition refers to all efficient prices, $p$, supporting the allocation $x$ at $u$, that is, to any $p \in \Pi(x, u)$, while our condition only refers to those efficient prices belonging to the set $\pi^{F}(x, u)$. So, the difference materializes only when $\pi^{F}(x, u)$ is a proper subset of $\Pi(x, u)$. In this sense, our forthrightness condition is slightly weaker than the regularity condition provided by Saijo et al. (1999), when it is applied to the standard framework of natural implementation. 
In line with the previous section, this section assumes that an agent involved in a pricequantity ${ }^{2}$ mechanism is truthful when only the consumption bundles component reported by this agent is consistent with the pre-specified welfare criterion for the underlying economy. Formally, for any economy $u \in U_{N}$, any societal goal $F \in \mathcal{F}$, and any agent $i \in N$, the set of truthful messages for a natural price-quantity ${ }^{2}$ mechanism, $\gamma$, is given by

$$
T_{i}^{\gamma}(u, F) \equiv\left\{\left(p, x_{i}, x_{i+1}\right) \in \Delta \times Q^{2} \mid \exists x_{-\{i, i+1\}} \in \mathbb{R}^{(n-2) \ell}:\left(x_{i}, x_{i+1}, x_{-\{i, i+1\}}\right) \in F(u)\right\} .
$$

Let us now turn to state a condition relevant to partially honest implementation by natural price-quantity ${ }^{2}$ mechanisms. To this end, let us first define a new necessary and sufficient condition for implementation by natural price-quantity ${ }^{2}$ mechanisms in the standard framework.

Condition $\mathrm{GM}^{*}\left(G M^{*}\right)$ : For all $u, u^{*} \in U_{N}$, all $x \in F(u)$, and all $p \in \pi^{F}(x, u)$, if $\Lambda_{i}^{F}(x, p) \subseteq L\left(x_{i}, u_{i}^{*}\right)$ for all $i \in N$, then $x \in F\left(u^{*}\right)$.

Note that this condition is slightly weaker than the condition $G M$ introduced by Saijo et al. (1999; p. 278). This results from restricting the application of condition GM to only efficient prices belonging to the set $\pi^{F}(x, u)$. Since our forthrightness condition is weaker than that presented in Saijo et al. (1999) for natural price-quantity ${ }^{2}$ mechanisms, condition $G M^{*}$ is a plausible weakening of condition $G M$. Confirmation of this assertion can be made by applying the class of natural mechanisms presented in Definition 5 to the conventional framework. Within this framework, it can be shown that any efficient $S C C F$ is implementable by a natural price-quantity ${ }^{2}$ mechanism - which encompasses the forthrightness condition of Definition 5 above - if and only if $F$ satisfies our variant of condition $G M$, that is, condition $G M^{*}$. A remarkable implication of this characterization is that the constrained Walrasian correspondence becomes implementable by a natural price-quantity ${ }^{2}$ mechanism (Refer to Section 6 for details). This result runs counter to the findings of Saijo et al. (1999), according to which this $S C C$ is not implementable by any natural mechanism, unless the extra condition of budget balance is posited. ${ }^{17}$

As discussed in Section 2, in our set-up, to be ensured of the implementability of $F$, the mechanism designer has to design a mechanism in such a way that, no matter what the current economy is and no matter who the partially honest participants are, only the $F$-optimal allocations are realized as the equilibrium outcomes of the devised game form. In particular, for any given economy, the designed mechanism must also implement the targeted welfare criterion $F$ under all conceivable sets of partially honest agents. Correspondingly, a requirement for the partially honest implementability of $F$ must also be applied to any conceivable set $H \in \mathcal{H}$ of partially honest participants. Having mentioned this point, let us introduce the following weaker variant of condition $G M^{*}$ for characterizing partially honest implementation by natural price-quantity ${ }^{2}$ mechanisms.

Weak Condition $\mathrm{GM}^{*}\left(\widehat{G M}^{*}\right)$ : For any given $H \in \mathcal{H}$, for all $u, u^{*} \in U_{N}$, all $x \in F(u)$, and all $p \in \pi^{F}(x, u)$, if $\Lambda_{i}^{F}(x, p) \subseteq L\left(x_{i}, u_{i}^{*}\right)$ for all $i \in N$ and $x \notin F\left(u^{*}\right)$, then there exists $h \in H$ such that $x_{h} \neq x_{h}^{*}$ or $x_{h+1} \neq x_{h+1}^{*}$ for all $x^{*} \in F\left(u^{*}\right)$, where $n+1=1$.

The following theorem (whose proof is relegated to the Appendix) shows that condition $\widehat{G M}^{*}$ is necessary and sufficient for partially honest implementation by natural pricequantity ${ }^{2}$ mechanisms.

\footnotetext{
${ }^{17}$ This $S C C$ is implementable by a natural price-quantity ${ }^{2}$ mechanism only when there are two commodities in the economy (Saijo et al., 1999; Lemma 2).
} 
Theorem 2. Let $n \geq 3$ and Assumption 1 hold. An SCC $F \in \mathcal{F}$ is partially honest implementable by a natural price-quantity ${ }^{2}$ mechanism if and only if it satisfies condition $\widehat{G M}^{*}$.

The above theorem, when combined with Theorem 1 and Corollary 1, shows that, when there are four or more participants, the class of $S C C$ s that are partially honest implementable by natural price-quantity ${ }^{2}$ mechanisms is not equivalent to the class of $S C C$ s that are partially honest implementable by natural price-allocation mechanisms (Refer to Section 6 for a more detailed discussion). This result stands in stark contrast to the equivalence result between implementation by natural price-allocation mechanisms and implementation by natural price-quantity ${ }^{2}$ mechanisms obtained by Saijo et al. (1996a; 1999).

\section{Price-quantity mechanisms}

From the viewpoint of decentralization, a price-quantity mechanism is qualitatively different from a price-quantity ${ }^{2}$ mechanism. Though a price-quantity ${ }^{2}$ mechanism is more efficient than a price-allocation mechanism, it is still far from the self-relevancy condition advocated by Hurwicz (1960). According to this condition, each agent must reveal information related only to her own characteristics. In this sense, no price-quantity ${ }^{2}$ mechanism is decentralized because each participant is required to disclose information about another participant. In contrast, if we consider Pareto efficiency to be one of our minimum requirements and regard the price announcement component of the message as part of the self-relevant information, then a natural price-quantity mechanism is a self-relevant and natural mechanism. This section, then, investigates partially honest implementability of efficient $S C C$ s by natural price-quantity mechanisms.

The definition of partially honest implementation by price-quantity mechanisms used in this section can be stated as follows.

Definition 6. An $S C C F \in \mathcal{F}$ is partially honest implementable by a natural price-quantity mechanism if there exists a mechanism $\gamma=(M, g)$ such that:

(i) $\gamma$ partially honestly implements $F$;

(ii) for each $i \in N, M_{i}=\Delta \times Q$, where $Q \equiv\left\{x_{i} \in \mathbb{R}_{+}^{\ell} \mid x_{i} \leq \Omega\right\}$;

(iii) for each $u \in U_{N}$, each $x \in F(u)$, and each $p \in \pi^{F}(x, u)$, if $m_{i}=\left(p, x_{i}\right)$ for each $i \in N$, then $m \in N E\left(\gamma, \succcurlyeq^{u}\right)$ and $g(m)=x$;

(iv) $\gamma$ is individually feasible and balanced;

(v) $\gamma$ satisfies the best response property.

Definition 6 stipulates individual feasibility, balancedness, and the best response property in a natural price-quantity mechanism. Moreover, Condition (iii) in Definition 6 represents the regularity condition of forthrightness in a natural price-quantity mechanism. It is important to note that this condition differs slightly from that postulated by Saijo et al. (1999; Definition 2(iii)). As in condition (iii) of Definition 5, condition (iii) of Definition 6 only refers to price vectors $p$ in the set $\pi^{F}(x, u)$, whereas the regularity condition of forthrightness in Saijo et al. (1999; Definition 2(iii)) refers to any price vector $p$ in $\Pi(x, u)$.

In line with the previous sections, we assume that a participant of a natural price-quantity mechanism is truthful when the consumption bundle reported by this agent coincides with a bundle that the $S C C$ dictates for her, given the underlying economy. Formally, for any economy $u \in U_{N}$, any societal goal $F \in \mathcal{F}$, and any agent $i \in N$, the set of truthful messages 
for a natural price-quantity mechanism $\gamma$ is given by

$$
T_{i}^{\gamma}(u, F) \equiv\left\{\left(p, x_{i}\right) \in \Delta \times Q \mid \exists x_{-i} \in \mathbb{R}^{(n-1) \ell}:\left(x_{i}, x_{-i}\right) \in F(u)\right\} .
$$

A first condition relevant to partially honest implementation by natural price-quantity mechanisms is stated below.

Weak Condition GM* $\left(\overline{G M}^{*}\right)$. For any given $H \in \mathcal{H}$, for all $u, u^{*} \in U_{N}$, all $x \in F(u)$, and all $p \in \pi^{F}(x, u)$, if $\Lambda_{i}^{F}(x, p) \subseteq L\left(x_{i}, u_{i}^{*}\right)$ for all $i \in N$, and $x \notin F\left(u^{*}\right)$, then, for some $h \in H, x_{h}^{*} \neq x_{h}$ for all $x^{*} \in F\left(u^{*}\right)$.

For the reasons illustrated in the previous section, condition $\overline{G M}^{*}$ is defined for any admissible set of partially honest agents $H \in \mathcal{H}$. It is important to note that condition $\overline{G M}^{*}$ implies condition $\widehat{G M}^{*}$.

Note that, as in the case of Saijo et al. (1996a; 1999), we cannot but impose another condition for implementation by natural price-quantity mechanisms, which should define a punishment scheme for potential deviators. Let $(p, x) \in \Delta \times A$ be given. Define the set $\bar{F}^{-1}(x, p)$ as follows:

$$
\bar{F}^{-1}(x, p) \equiv \begin{cases}F_{!}^{-1}(x, p) & \text { if } F_{!}^{-1}(x, p) \neq \varnothing, \\ F^{-1}(x, p) & \text { if } F_{!}^{-1}(x, p)=\varnothing, \text { and } \nexists p^{\prime} \in \Delta: F_{!}^{-1}\left(x, p^{\prime}\right) \neq \varnothing .\end{cases}
$$

Then, let $I^{F}(p, x) \equiv\left\{i \in N \mid \bar{F}^{-1}\left(\left(\Omega-\sum_{j \neq i} x_{j}, x_{-i}\right), p\right) \neq \varnothing\right\}$ denote the set of potential deviators for price-quantity implementation. The following condition is a slightly weaker variant of the condition GPQ introduced by Saijo et al. (1999; p. 279).

Condition $\mathrm{GPQ}^{*}\left(G P Q^{*}\right)$. For any $(p, x) \in \Delta \times Q^{n}$ such that $I^{F}(p, x)=N$ and $x \notin A$, there exists $z(p, x) \in A$ such that:

(i) $z_{i}(p, x) \in \Lambda_{i}^{F}\left(\left(\Omega-\sum_{l \neq i} x_{l}, x_{-i}\right), p\right)$ for all $i \in N$;

(ii) for all $u^{*} \in U_{N}$, if $\Lambda_{i}^{F}\left(\left(\Omega-\sum_{l \neq i} x_{l}, x_{-i}\right), p\right) \subseteq L\left(z_{i}(p, x), u_{i}^{*}\right)$ for all $i \in N$, then $z(p, x) \in F\left(u^{*}\right)$.

The only difference between condition $G P Q$ and $G P Q^{*}$ lies in the definition of the set of potential deviators, $I^{F}(p, x)$. While condition $G P Q$ defines it by referring to $F^{-1}$, the above condition defines the set $I^{F}(p, x)$ by referring to $\bar{F}^{-1}$. Moreover, it can be shown that condition $G P Q^{*}$, when combined with condition $G M^{*}$, fully identifies the class of $S C C$ s that are implementable by natural price-quantity mechanisms in the standard set-up. A remarkable consequence of this characterization is that the constrained Walrasian correspondence is implementable by a natural price-quantity mechanism presented in Definition 6 (Refer to Section 6 for details), while its implementability is impossible under the definition of natural price-quantity mechanism provided by Saijo et al. (1999; Definition 2).

Next, let us introduce a variant of $G P Q^{*}$ to characterize partially honest implementation by natural price-quantity mechanisms. Again, note that the requirement of the axiom should be applied to any given potential set $H \in \mathcal{H}$ of partially honest agents.

Weak Condition GPQ $\left(\overline{G P Q}^{*}\right)$. For any given $H \in \mathcal{H}$, and any $(p, x) \in \Delta \times Q^{n}$ such that $I^{F}(p, x)=N$ and $x \notin A$, there exists $z(p, x) \in A$ such that:

(i) $z_{i}(p, x) \in \Lambda_{i}^{F}\left(\left(\Omega-\sum_{l \neq i} x_{l}, x_{-i}\right), p\right)$ for all $i \in N$;

(ii) for each $i \in N$, there exists a map $S_{i}\left(\cdot ;\left(p, x_{-i}\right)\right): Q \rightarrow Q$ such that, for all $\left(p^{\prime}, x_{i}^{\prime}\right) \in \Delta \times$ $Q$, if there exist $u^{\prime} \in U_{N}$ and $x_{i}^{*} \in Q$ such that $I^{F}\left(p, x^{*}\right)=N, \Lambda_{j}^{F}\left(\left(\left(\Omega-\sum_{l \neq j} x_{l}^{*}\right), x_{-j}^{*}\right), p\right) \subseteq$ 
$L\left(z_{j}\left(p, x^{*}\right), u_{j}^{\prime}\right)$ for all $j \in N$, where $x^{*} \equiv\left(x_{i}^{*}, x_{-i}\right)$, and $\left(x_{i}^{\prime}, \hat{x}_{-i}\right) \in F\left(u^{\prime}\right)$ for some $\hat{x}_{-i} \in \mathbb{R}^{(n-1) \ell}$, then $S_{i}\left(\left(p^{\prime}, x_{i}^{\prime}\right) ;\left(p, x_{-i}\right)\right) \in \Lambda_{i}^{F}\left(\left(\Omega-\sum_{l \neq i} x_{l}, x_{-i}\right), p\right)$ holds; otherwise, $S_{i}\left(\left(p^{\prime}, x_{i}^{\prime}\right) ;\left(p, x_{-i}\right)\right)=\mathbf{0}$;

(iii) for all $u^{*} \in U_{N}$, if $\Lambda_{i}^{F}\left(\left(\Omega-\sum_{l \neq i} x_{l}, x_{-i}\right), p\right) \subseteq L\left(z_{i}(p, x), u_{i}^{*}\right)$ for all $i \in N$ and $z(p, x) \notin F\left(u^{*}\right)$, then there exists $h \in H$ such that:

(a) $\left(x_{h}, \hat{x}_{-h}\right) \notin F\left(u^{*}\right)$ for all $\hat{x}_{-h} \in \mathbb{R}^{(n-1) \ell}$, and

(b) there exists $\left(p^{\prime \prime}, x_{h}^{\prime \prime}\right) \in \Delta \times Q$, with $\left(x_{h}^{\prime \prime}, \hat{x}_{-h}\right) \in F\left(u^{*}\right)$ for some $\hat{x}_{-h} \in \mathbb{R}^{(n-1) \ell}$, such that $S_{h}\left(\left(p^{\prime \prime}, x_{h}^{\prime \prime}\right) ;\left(p, x_{-h}\right)\right) \in \partial L\left(z_{h}(p, x), u_{h}^{*}\right)$.

Like condition $G P Q$, in condition $\overline{G P Q}^{*}$ the punishment allocation $z(p, x)$ depends on the profile $(p, x)$. Saijo et al. (1996b) introduced a punishment condition - named simple punishment condition - that requires the same feasible punishment outcome in all cases in which all participants are potential deviators. Since a variant of our theorem below can easily be obtained by employing a weaker variant of Saijo et al. (1996b)'s simple punishment condition, we omit it here for the sake of brevity. ${ }^{18}$

The following theorem (whose proof is relegated to the Appendix) shows that condition $\overline{G M}^{*}$ and condition $\overline{G P Q}^{*}$ fully identify the class of SCCs that are partially honest implementable by natural price-quantity mechanisms.

Theorem 3. Let $n \geq 3$ and suppose that Assumption 1 holds. An SCC $F \in \mathcal{F}$ is partially honest implementable by a natural price-quantity mechanism if and only if it satisfies condition $\overline{G M}^{*}$ and condition $\overline{G P Q}^{*}$.

If balancedness is replaced by the less demanding postulate of the weak balancedness, in the sense that the mechanism is required to be only weakly balanced, then condition $\overline{G P Q}^{*}$ can be dispensed with from Theorem 3, as the following corollary shows.

Corollary 3. Let $n \geq 3$ and suppose that Assumption 1 holds. Let $F \in \mathcal{F}$. If free disposal is allowed, then $F$ is partially honest implementable by a price-quantity mechanism satisfying forthrightness, individual feasibility, weak balancedness and the best response property if and only if it satisfies condition $\overline{G M}^{*}$.

\section{Implications}

In this section, we discuss briefly some implications of the findings of the previous sections.

Before going into detail, let us specify the structure on which the analysis is based. Firstly, the informational assumption on which our theorems are based is that the mechanism designer knows that there are partially honest participants and how these agents behave. Then, the mechanism designer does not know the identity of these agents or their exact number. Due to this truly limited information, the mechanism designer, to be ensured of the implementability of her goal $F \in \mathcal{F}$, has to design a mechanism in such a way that, regardless of the current economy and the partially honest participants involved in the mechanism, only

\footnotetext{
${ }^{18}$ The weaker variant of Saijo et al. (1996b)'s simple punishment condition can be stated as follows: For any given $H \in \mathcal{H}$, and for all $(p, x) \in \Delta \times Q^{n}$ such that $I^{F}(p, x)=N$ and $x \notin A$, there exists $z \in A$ such that: (i) $z_{i} \in \Lambda_{i}^{F}\left(\left(\Omega-\sum_{l \neq i} x_{l}, x_{-i}\right), p\right)$ for all $i \in N$; (ii) for all $u^{*} \in U_{N}$, if $\Lambda_{i}^{F}\left(\left(\Omega-\sum_{l \neq i} x_{l}, x_{-i}\right), p\right) \subseteq L\left(z_{i}, u_{i}^{*}\right)$ for all $i \in N$ and $z \notin F\left(u^{*}\right)$, then, for some $h \in H, x_{h} \neq x_{h}^{*}$ for all $x^{*} \in F\left(u^{*}\right)$. Accordingly, the pricequantity mechanism devised in the proof of Theorem 3 below (See the Appendix) will require only minor changes to obtain the desired result.
} 
the $F$-optimal outcomes emerge as the equilibrium outcomes of the devised game form. This implies that the mechanism designer has to devise a mechanism that guarantees the partially honest implementability of $F$ even when $\mathcal{H}=2^{N} \backslash \varnothing$. Indeed, by covering this case, the mechanism designer is ensured of the implementability of $F$ for any other specialization that the set $\mathcal{H}$ may take. For this reason, in what follows, we turn to analyze some implications of the above theorems under the specification that $\mathcal{H}=2^{N} \backslash \varnothing$. Secondly, the welfare criteria $F$ considered below are defined on the domain $\bar{U}$ of all profiles of utility functions which are continuous, quasi-concave, and strictly monotonic. Finally, the analysis is limited to the following well-known welfare criteria.

Pareto correspondence $P: P(u)=\left\{x \in A \mid\right.$ there is no $y \in A: u_{i}\left(y_{i}\right)>u_{i}\left(x_{i}\right)$ for all $i \in N\}$.

Individual rational and efficient correspondence $\operatorname{Ir} P: \operatorname{Ir} P(u) \equiv\left\{x \in A \mid u_{i}\left(x_{i}\right) \geq u_{i}\left(\omega_{i}\right)\right.$ for all $i \in N\} \cap P(u)$.

Egalitarian-equivalent and efficient correspondence $E E: E E(u) \equiv\{x \in A \mid$ there is a unique maximal number $\lambda \in(0,1): u_{i}\left(x_{i}\right)=u_{i}(\lambda \Omega)$ for all $\left.i \in N\right\} \cap P(u)$.

No-envy and efficient correspondence $N P: N P(u) \equiv\left\{x \in A \mid u_{i}\left(x_{i}\right) \geq u_{i}\left(x_{j}\right)\right.$ for all $i, j \in N\} \cap P(u)$.

Walrasian correspondence $W: W(u) \equiv\left\{x \in A \mid\right.$ there is $p \in \Delta: p \cdot x_{i}=p \cdot \omega_{i}$ for all $i \in N$, and for any $y_{i} \in \mathbb{R}_{+}^{\ell}$ with $\left.p \cdot y_{i} \leq p \cdot \omega_{i}: u_{i}\left(y_{i}\right) \leq u_{i}\left(x_{i}\right)\right\}$.

Constrained Walrasian correspondence $W_{c}: W_{c}(u) \equiv\left\{x \in A \mid\right.$ there is $p \in \Delta: p \cdot x_{i}=p \cdot \omega_{i}$ for all $i \in N$, and for any $y_{i} \in \mathbb{R}_{+}^{\ell}$ with $y_{i} \leq \Omega$ and $\left.p \cdot y_{i} \leq p \cdot \omega_{i}: u_{i}\left(y_{i}\right) \leq u_{i}\left(x_{i}\right)\right\}$.

The first result is an impossibility one, since it shows that condition $\widehat{G M}^{*}$ imposes nontrivial restrictions on the class of partially honest implementable $S C C$ s by natural pricequantity $^{2}$ mechanisms.

Corollary 4. Let $n \geq 4$ and suppose that Assumption 1 holds with $\mathcal{H}=2^{N} \backslash \varnothing$. Then, the egalitarian-equivalent and efficient correspondence, $E E$, defined on $U_{N}=\bar{U}$, is not partially honest implementable by any natural price-quantity ${ }^{2}$ mechanism.

Proof. Suppose that Assumption 1 holds with $\mathcal{H}=2^{N} \backslash \varnothing$. Moreover, let $n \geq 4$. By Theorem 2, it suffices to show that $E E$, defined on $U_{N}=\bar{U}$, does not satisfy condition $\widehat{G M}^{*}$. To this end, take any $u \in U_{N}$, where each $u_{i}$ is strictly concave and differentiable, and any $x \in E E(u) \cap \mathbb{R}_{++}^{n \ell}$. Then, there exists a unique supporting price $\{p\}=\pi^{E E}(x, u)$, for which each $\Lambda_{i}^{E E}(x, p)$ is constructed. By definition, for each $u^{\prime} \in E E^{-1}(x, p)$, there exists $\lambda^{u^{\prime}} \in(0,1)$ such that, for each $i \in N, u_{i}\left(x_{i}\right)=u_{i}\left(\lambda^{u^{\prime}} \Omega\right)$. In addition, for any $\lambda^{\prime}>\lambda^{u^{\prime}}$, if there exists $x^{\prime} \in \mathbb{R}_{+}^{n \ell}$ such that $u_{i}\left(x_{i}^{\prime}\right)=u_{i}\left(\lambda^{\prime} \Omega\right)$ for each $i \in N$, then $\sum_{i \in N} x_{i}^{\prime}>\Omega$. Hence, $\lambda^{u^{\prime}} \Omega \in \partial L\left(x_{i}, u_{i}^{\prime}\right)$ for each $i \in N$. Let $\lambda^{(x, p)} \equiv \min _{u^{\prime} \in E E^{-1}(x, p)} \lambda^{u^{\prime}}$. Then, $\lambda^{(x, p)} \Omega \in$ $\partial \Lambda_{i}^{E E}(x, p)$ for each $i \in N$. Moreover, each $\Lambda_{i}^{E E}(x, p)$ has the following property: for each $i \in N$, there is a neighborhood $B\left(x_{i}\right) \subset \partial \Lambda_{i}^{E E}(x, p)$ of $x_{i}$ such that, for any $y_{i} \in B\left(x_{i}\right)$, $p \cdot y_{i}=p \cdot x_{i}$.

Take an economy $u^{*} \in U_{N}$, where $u_{i}^{*}$ is strictly concave and differentiable for each agent $i \in N$, such that $\Lambda_{i}^{E E}(x, p) \subseteq L\left(x_{i}, u_{i}^{*}\right)$ for all $i \in N$. Moreover, suppose that there are two distinct agents $j, k \in N$ such that, for some $\epsilon>0, u_{j}^{*}\left(x_{j}+(\epsilon, \mathbf{0})\right)=u_{j}^{*}\left(\lambda^{*} \Omega\right)$ and $u_{k}^{*}\left(x_{k}-(\epsilon, \mathbf{0})\right)=u_{k}^{*}\left(\lambda^{*} \Omega\right)$ for some $\lambda^{*}>\lambda^{(x, p)}$, and $D u_{j}^{*}\left(x_{j}+(\epsilon, \mathbf{0})\right)=p=D u_{k}^{*}\left(x_{k}-(\epsilon, \mathbf{0})\right)$, where $\mathbf{0}$ is the $\ell-1$-th dimensional zero vector. Finally, for any other agent $l \in N \backslash\{j, k\}$, 
let $u_{l}^{*}\left(x_{l}\right)=u_{l}^{*}\left(\lambda^{*} \Omega\right)$. By construction, there exist $\lambda^{j}, \lambda^{k}$ such that $\lambda^{(x, p)}<\lambda^{j}<\lambda^{*}<\lambda^{k}$, $u_{j}^{*}\left(x_{j}\right)=u_{j}^{*}\left(\lambda^{j} \Omega\right)$, and $u_{k}^{*}\left(x_{k}\right)=u_{k}^{*}\left(\lambda^{k} \Omega\right)$. Note that such a profile $u^{*}$ exists because of our supposition that $U_{N}=\bar{U}$. Thus, $x \in P\left(u^{*}\right) \backslash E E\left(u^{*}\right)$, but $x^{*} \equiv\left(x_{j}+(\epsilon, \mathbf{0}), x_{k}-(\epsilon, \mathbf{0}), x_{-\{j, k\}}\right) \in$ $E E\left(u^{*}\right)$. Note that, since $E E$ is essentially single-valued, ${ }^{19}$ and each $u_{i}^{*}$ is strictly concave, $\left\{x^{*}\right\}=E E\left(u^{*}\right)$ holds.

Consider a permutation $\phi$ such that $\phi(k)=\phi(j)+1, \phi(j+1)=k$, and $\phi(l)=l$ for any $l \in N \backslash\{j+1, k\}$. Correspondingly, let $u^{* \phi} \equiv\left(u_{\phi(l)}^{*}\right)_{l \in N}, x^{* \phi} \equiv\left(x_{\phi(l)}^{*}\right)_{l \in N}, u^{\phi} \equiv\left(u_{\phi(l)}\right)_{l \in N}$, and $x^{\phi} \equiv\left(x_{\phi(l)}\right)_{l \in N}$. Then, since $E E$ meets the anonymity property, $x^{* \phi} \in E E\left(u^{* \phi}\right)$ but $x^{\phi} \in E E\left(u^{\phi}\right) \backslash E E\left(u^{* \phi}\right)$. Observe that, by construction, $x_{\phi(j)}^{\phi} \neq x_{\phi(j)}^{* \phi}, x_{\phi(k)}^{\phi} \neq x_{\phi(k)}^{* \phi}$, and $x_{\phi(l)}^{\phi}=x_{\phi(l)}^{* \phi}$ for any other $\phi(l) \in N \backslash\{\phi(j), \phi(k)\}$. Moreover, since $\mathcal{H}=2^{N} \backslash \varnothing$ and $n \geq 4$, there exists $H \in \mathcal{H}$ such that $\{\phi(j), \phi(j)-1, \phi(k)\} \cap H=\varnothing$. Thus, for any $h \in H$, $x_{h}^{\phi}=x_{h}^{* \phi}$ and $x_{h+1}^{\phi}=x_{h+1}^{* \phi}$ hold, which implies that $E E$ does not satisfy condition $\widehat{G M}^{*}$, as sought.

Note that this corollary, combined with Theorem 1 and Corollary 1, implies that, when there are four or more participants, the class of $S C C$ s that are partially honest implementable by natural price-allocation mechanisms differs from the class of $S C C$ s that are partially honest implementable by natural price-quantity ${ }^{2}$ mechanisms. This result runs counter to the equivalence relationship holding between these two classes in the conventional natural implementation framework.

While it is easy to see that the individual rational and efficient correspondence, $\operatorname{Ir} P$, and the no-envy and efficient correspondence, $N P$, satisfy condition $\widehat{G M}^{*}$, it is not apparent that the constrained Walrasian correspondence, $W_{c}$, is partially honest implementable by a natural price-quantity ${ }^{2}$ mechanism. This is due to the fact that Saijo et al. (1999; Lemma 3 ) show that $W_{c}$ is not implementable by any natural mechanism in economies endowed with more than two commodities. In contrast to this impossibility result, the following lemma shows that this $S C C$ can be implemented by a natural price-quantity ${ }^{2}$ mechanism under the concept of natural implementation presented in Definition 5.

Lemma 1. Let $n \geq 3$. Then, the constrained Walrasian correspondence, $W_{c}$, defined on $U_{N}=\bar{U}$, satisfies condition $G M^{*}$.

Proof. Let $n \geq 3$. Assume that $W_{c}$ is defined on $U_{N}=\bar{U}$, and take any $u \in U_{N}$ and any $x \in W_{c}(u)$. Let us first show that $\Pi^{W_{c}}(x, u)$ is non-empty and consists solely of the constrained Walrasian competitive equilibrium price vectors. Then, let $p^{W_{c}} \in \Pi(x, u)$ be a constrained Walrasian price vector. Note that since $x \in W_{c}(u)$, there is such a price vector. Then, by definition, $p^{W_{c}} \cdot x_{i}=p^{W_{c}} \cdot \omega_{i}$ for any $i \in N$. Since $U_{N}=\bar{U}$, there always exists $u^{\prime} \in U_{N}$ such that $\left\{p^{W_{c}}\right\}=\Pi\left(x, u^{\prime}\right)$. Then, by the definition of $W_{c}, x \in W_{c}\left(u^{\prime}\right)$, which, in turn, implies that $W_{c !}^{-1}\left(x, p^{W_{c}}\right) \neq \varnothing$. Therefore, $p^{W_{c}} \in \Pi^{W_{c}}(x, u)$, as sought. Next, let $p \in \Pi(x, u)$ be not a constrained Walrasian price vector. Then, by definition, $p \cdot x_{i} \neq p \cdot \omega_{i}$ for some $i \in N$. Since $U_{N}=\bar{U}$, there always exists $u^{\prime} \in U_{N}$ such that $\{p\}=\Pi\left(x, u^{\prime}\right)$. This implies that $x \in P\left(u^{\prime}\right)$. Then, since $p \cdot x_{i} \neq p \cdot \omega_{i}$ holds for some $i \in N, x \notin W_{c}\left(u^{\prime}\right)$. The same argument is applied for any other $u^{\prime \prime} \in U_{N}$ such that $\{p\}=\Pi\left(x, u^{\prime \prime}\right)$. Thus, $W_{c !}^{-1}(x, p)=\varnothing$, which, in turn, implies $p \notin \Pi^{W_{c}}(x, u)$, as sought. Therefore, $\pi^{W_{c}}(x, u)=\Pi^{W_{c}}(x, u)$ holds for any $u \in U_{N}$ and any allocation $x$ such that

${ }^{19}$ An SCC $F$ is essentially single-valued if, for any $u \in U_{N}, x, x^{\prime} \in F(u)$ implies that $u_{i}\left(x_{i}\right)=u_{i}\left(x_{i}^{\prime}\right)$ for all $i \in N$. 
$x \in W_{c}(u)$. It follows that for any $u \in U_{N}$, any $x \in W_{c}(u)$, any $p \in \pi^{W_{c}}(x, u)$, and any $i \in N, \Lambda_{i}^{W_{c}}(x, p)=\cap_{u^{\prime} \in W_{c}^{-1}(x, p)} L\left(x_{i}, u_{i}^{\prime}\right)=\left\{y_{i} \in \mathbb{R}_{+}^{\ell} \mid y_{i} \leq \Omega\right.$ and $\left.p \cdot y_{i}=p \cdot \omega_{i}\right\}$ holds. Then, if $u^{*} \in U_{N}$ is such that $\Lambda_{i}^{W_{c}}(x, p) \subseteq L\left(x_{i}, u_{i}^{*}\right)$ for all $i \in N$, then it is easy to see that $x \in W_{c}\left(u^{*}\right)$. This implies that $W_{c}$ defined on $\bar{U}$ satisfies condition $G M^{*}$.

The above lemma, combined with Theorem 2, readily yields the following corollary.

Corollary 5. Let $n \geq 3$ and suppose that Assumption 1 holds. Then, the constrained Walrasian correspondence, $W_{c}$, defined on $U_{N}=\bar{U}$, is partially honest implementable by $a$ natural price-quantity ${ }^{2}$ mechanism.

The next result is another possibility result, because it shows that the Walrasian correspondence, $W$, is partially honest implementable by a natural price-quantity ${ }^{2}$ mechanism in two-commodity economies. This stands in stark contrast with the standard literature on implementation, where it is well-known that this $S C C$ is not implementable. ${ }^{20}$

Lemma 2. Let $n \geq 3$ and $\ell=2$. Suppose that Assumption 1 holds. Then, the Walrasian correspondence, $W$, defined on $U_{N}=\bar{U}$, satisfies condition $\overline{G M}^{*}$.

Proof. Suppose that $n \geq 3$ and $\ell=2$. Moreover, assume that $W$ is defined on $U_{N}=\bar{U}$, and take any $u \in U_{N}$ and any $x \in W(u)$. Then, let us first show that $\Pi^{W}(x, u)$ is non-empty and consists solely of the Walrasian competitive equilibrium price vectors. Let $p^{W} \in \Pi(x, u)$ be a Walrasian price vector. Note that since $x \in W(u)$, there is such a price vector. Then, by definition, $p^{W} \cdot x_{i}=p^{W} \cdot \omega_{i}$ for any $i \in N$. Since $U_{N}=\bar{U}$, there always exists $u^{\prime} \in U_{N}$ such that $\left\{p^{W}\right\}=\Pi\left(x, u^{\prime}\right)$. Then, by the definition of $W, x \in W\left(u^{\prime}\right)$, which, in turn, implies that $W_{!}^{-1}\left(x, p^{W}\right) \neq \varnothing$. Thus, $p^{W} \in \Pi^{W}(x, u)$. Next, let $p \in \Pi(x, u)$ be not a Walrasian price vector. Then, by definition, $p \cdot x_{i} \neq p \cdot \omega_{i}$ for some $i \in N$. Since $U_{N}=\bar{U}$, there always exists $u^{\prime} \in U_{N}$ such that $\{p\}=\Pi\left(x, u^{\prime}\right)$. This implies that $x \in P\left(u^{\prime}\right)$, and $p$ is the unique supporting price for $u^{\prime}$ at $x$. Then, since $p \cdot x_{i} \neq p \cdot \omega_{i}$ holds for some $i \in N$, $x \notin W\left(u^{\prime}\right)$. The same argument is applied for any other $u^{\prime \prime} \in U_{N}$ such that $\{p\}=\Pi\left(x, u^{\prime \prime}\right)$. Thus, $W_{!}^{-1}(x, p)=\varnothing$, which, in turn, implies $p \notin \Pi^{W}(x, u)$. In summary, $\Pi^{W}(x, u)$ is nonempty and consists solely of the Walrasian price vectors. Given this, $\pi^{W}(x, u)=\Pi^{W}(x, u)$ holds for any $u \in U_{N}$ and any allocation $x$ such that $x \in W(u)$. Then, for any $u \in U_{N}$, any $x \in W(u)$, and any $p \in \pi^{W}(x, u)$, we have that $\Lambda_{i}^{W}(x, p)=\cap_{u^{\prime} \in W^{-1}(x, p)} L\left(x_{i}, u_{i}^{\prime}\right)=$ $\left\{y_{i} \in \mathbb{R}_{+}^{\ell} \mid y_{i} \leq \Omega\right.$ and $\left.p \cdot y_{i}=p \cdot \omega_{i}\right\}$ for any $i \in N$. Finally, suppose that $u^{*} \in U_{N}$ is such that $\Lambda_{i}^{W}(x, p) \subseteq L\left(x_{i}, u_{i}^{*}\right)$ for all $i \in N$, but $x \notin W\left(u^{*}\right)$. Then, for any $p \in \Delta$ such that $p \cdot x_{i}=p \cdot \omega_{i}$ for all $i \in N$, there exists $j \in N$ such that $x_{j} \notin \arg \max _{p \cdot y_{j}=p \cdot \omega_{j}} u_{j}^{*}\left(y_{j}\right)$. Since $\Lambda_{i}^{W}(x, p) \subseteq L\left(x_{i}, u_{i}^{*}\right)$ for all $i \in N, x$ is not an interior allocation, and $x_{j}^{\prime} \not \leq \Omega$ if $x_{j}^{\prime} \in \arg \max _{p \cdot y_{j}=p \cdot \omega_{j}} u_{j}^{*}\left(y_{j}\right)$. Thus, for such a price vector $p$, there is no associated Walrasian allocation under $u^{*}$. Hence, when $\ell=2$, for any $x^{*} \in W\left(u^{*}\right)$, the associated Walrasian price vector $p^{*}$ implies that, for each $i \in N, p^{*} \cdot x_{i} \neq p^{*} \cdot \omega_{i}$. This, in turn, implies that, for any $x^{*} \in W\left(u^{*}\right), x_{i}^{*} \neq x_{i}$ for each $i \in N$. It follows that $x_{h}^{*} \neq x_{h}$ for any $H \in \mathcal{H}$ and any $h \in H$. We conclude that $W$, defined on $\bar{U}$, satisfies condition $\overline{G M}^{*}$.

Because condition $\overline{G M}^{*}$ implies condition $\widehat{G M}^{*}$, the following corollary is easily obtained from Lemma 2 and Theorem 2.

Corollary 6. Let $n \geq 3$ and $\ell=2$. Suppose that Assumption 1 holds. Then, the Walrasian correspondence, $W$, defined on $U_{N}=\bar{U}$, is partially honest implementable by a natural price-quantity ${ }^{2}$ mechanism.

\footnotetext{
${ }^{20}$ See, for instance, Jackson (2001).
} 
Note that, even under the supposition that an economy is endowed with only two commodities, the Walrasian correspondence is not partially honest implementable by any $s$-mechanism (see, Lombardi and Yoshihara, 2011a). In the light of this negative result, Corollary 6 seems remarkably surprising.

In the remaining part of this section, we discuss briefly some implications of Theorem 3; consequently, our discussion will focus on the condition $\overline{G M}^{*}$ and the condition $\overline{G P Q}^{*}$. Firstly, note that the individual rational and efficient correspondence, $\operatorname{Ir} P$, is partially honest implementable by a natural price-quantity mechanism, since it satisfies the conditions $G M^{*}$ and $G P Q^{*}$. Secondly, in two-commodity economies, the Walrasian correspondence, $W$, is still partially honest implementable by a natural price-quantity mechanism when Lemma 2 is read in combination with the following lemma.

Lemma 3. Let $n \geq 3$. Then, the Walrasian correspondence, $W$, defined on $U_{N}=\bar{U}$, satisfies condition $G P Q^{*}$.

Proof. Suppose that $n \geq 3$. Moreover, let $W$ be defined on $U_{N}=\bar{U}$. We show that $W$ satisfies condition $G P Q^{*}$. Take any $(p, x) \in \Delta \times Q^{n}$ such that $I^{W}(p, x)=N$ and $x \notin A$. Then, $W_{!}^{-1}\left(\left(\Omega-\sum_{j \neq i} x_{j}, x_{-i}\right), p\right) \neq \varnothing$ for each $i \in N$, which implies that for any $u \in W_{!}^{-1}\left(\left(\Omega-\sum_{j \neq i} x_{j}, x_{-i}\right), p\right), u_{i}\left(\Omega-\sum_{j \neq i} x_{j}\right) \geq u_{i}\left(\omega_{i}\right)$ holds for each $i \in N$. Thus, $\omega_{i} \in \Lambda_{i}^{W}\left(\left(\Omega-\sum_{j \neq i} x_{j}, x_{-i}\right), p\right)$ holds for all $i \in N$. Let $z(p, x) \equiv \omega$. Then, $W$ satisfies condition $G P Q^{*}(\mathrm{i})$. Moreover, for any $u^{*} \in U_{N}$, if $\Lambda_{i}^{W}\left(\left(\Omega-\sum_{j \neq i} x_{j}, x_{-i}\right), p\right) \subseteq$ $L\left(z_{i}(p, x), u_{i}^{*}\right)$, then $z_{i}(p, x) \in \arg \max _{p^{\prime} \cdot y_{i} \leq p^{\prime} \cdot \omega_{i}} u_{i}^{*}\left(y_{i}\right)$ holds for any $p^{\prime} \in \Delta$ and any $i \in N$, which, in turn, implies that $z(p, x) \in W\left(u^{*}\right)$. Therefore, $W$ satisfies condition $G P Q^{*}(i i)$. This completes the proof.

Thirdly, we find that the constrained Walrasian correspondence is partially honest implementable by a natural price-quantity mechanism, as the following result shows.

Lemma 4. Let $n \geq 3$. Then, the constrained Walrasian correspondence, $W_{c}$, defined on $U_{N}=\bar{U}$, satisfies condition $G P Q^{*}$.

Proof. Let $n \geq 3$. Since $W_{c} \subseteq W$, Lemma 3 implies that $W_{c}$ satisfies condition $G P Q^{*}$.

From the above lemmata, the following corollary is readily obtained.

Corollary 7. Let $n \geq 3$ and suppose that Assumption 1 holds. Then:

(i) The Walrasian correspondence, $W$, defined on $U_{N}=\bar{U}$, is partially honest implementable by a natural price-quantity mechanism when $\ell=2$;

(ii) The constrained Walrasian correspondence, $W_{c}$, defined on $U_{N}=\bar{U}$, is partially honest implementable by a natural price-quantity mechanism.

Finally, we show that the condition $\overline{G M}^{*}$ and the condition $\overline{G P Q}^{*}$ impose non-trivial restrictions on the class of partially honest implementable $S C C$ s by natural price-quantity mechanisms.

Lemma 5. Let $n \geq 3$ and suppose that Assumption 1 holds. Then, the Pareto correspondence, $P$, defined on $U_{N}=\bar{U}$, does not satisfy condition $\overline{G P Q}^{*}$ (i).

Proof. This is due to the fact that the Pareto correspondence does not satisfy condition $P Q(\mathrm{i})$, as shown in Saijo et al. (1996a). 


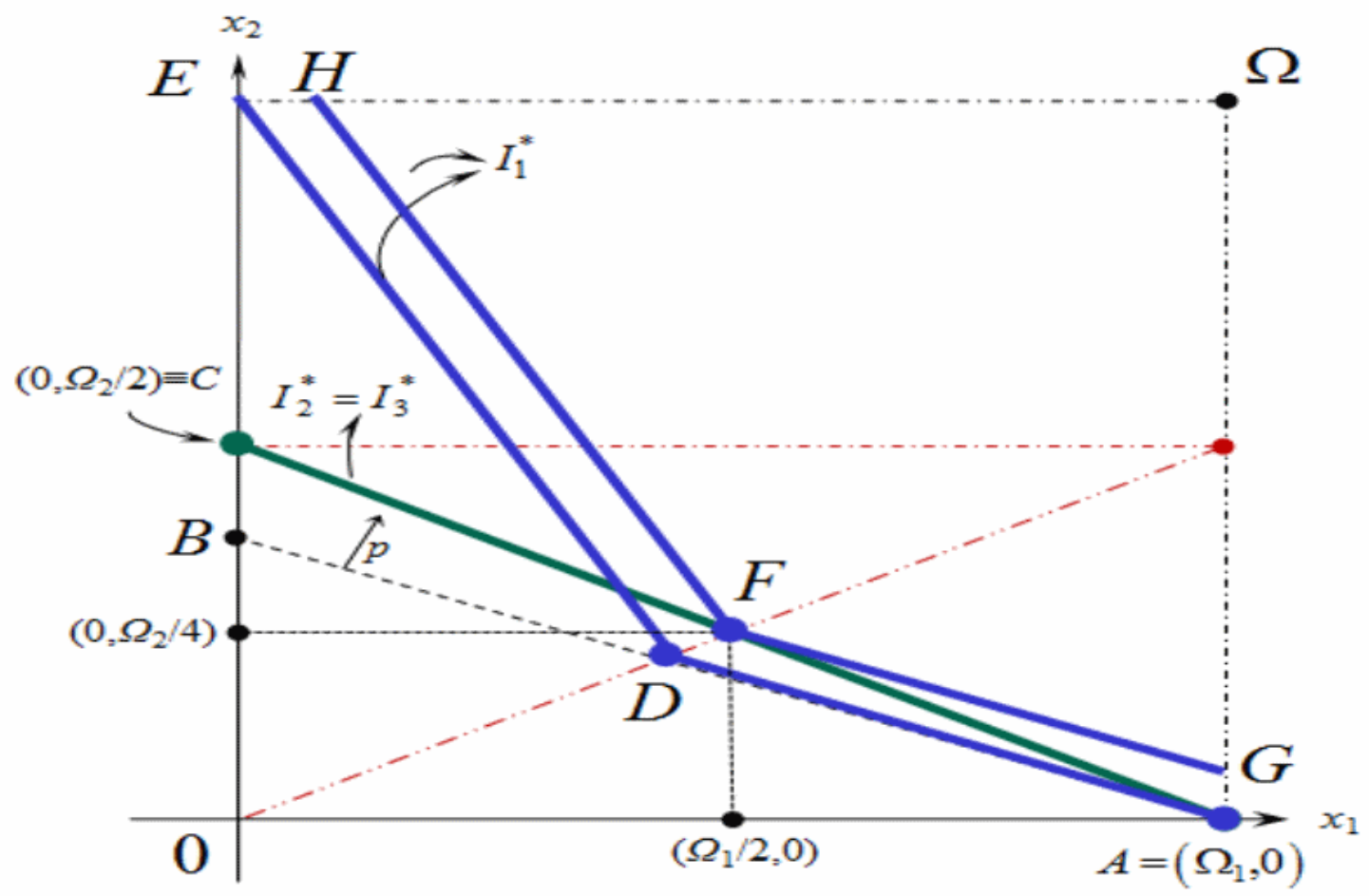

FIG. 1. $N P$ does not satisfy condition $\overline{G P Q}$ *

Lemma 6. Let $n \geq 3$ and suppose that Assumption 1 holds with $\mathcal{H}=2^{N} \backslash \varnothing$. Then, the no-envy and efficient correspondence, $N P$, defined on $U_{N}=\bar{U}$, does not satisfy condition $\overline{G P Q}^{*}($ iii).

Proof. Let $n \geq 3$. Suppose that Assumption 1 holds with $\mathcal{H}=2^{N} \backslash \varnothing$. We show that $N P$ violates condition $\overline{G P Q}^{*}$ (iii) when it is defined on $U_{N}=\bar{U}$. To this end, let us consider the economy depicted in Figure 1 and Figure 2 of Saijo et al. (1999; Proof of Lemma 1). We reproduce this economy in Figure 1 below. Suppose that there are only three participants and two commodities. Assume that each participant $i$ announces $\left(p, x_{i}\right)$, with $x_{i}=\left(0, \frac{\Omega_{2}}{2}\right)$. Thus, $I^{N P}(p, x)=\{1,2,3\}$ and, for each agent $i, \Omega-x_{j}-x_{k}=\left(\Omega_{1}, 0\right)$, with $i \neq j, k$, and $\Lambda_{i}^{N P}\left(\left(\left(\Omega_{1}, 0\right), x_{-i}\right), p\right)$ is represented by the area of the Figure $\mathbf{0} A B$ plus the line $B C$. Following the argument in Saijo et al. (1999; Proof of Lemma 1), the allocation $z(p, x)$ which satisfies condition $\overline{G P Q}^{*}(\mathrm{i})$ is only within the set

$\left\{\left(\left(\Omega_{1}, 0\right),\left(0, \frac{\Omega_{2}}{2}\right),\left(0, \frac{\Omega_{2}}{2}\right)\right),\left(\left(0, \frac{\Omega_{2}}{2}\right),\left(\Omega_{1}, 0\right),\left(0, \frac{\Omega_{2}}{2}\right)\right),\left(\left(0, \frac{\Omega_{2}}{2}\right),\left(0, \frac{\Omega_{2}}{2}\right),\left(\Omega_{1}, 0\right)\right)\right\}$.

Given this situation, let us show that $N P$ violates condition $\overline{G P Q}^{*}$ (iii). Assume, to the contrary, that $N P$ satisfies condition $\overline{G P Q}^{*}$ (iii). Without loss of generality, let us focus on the case that $z(p, x)=\left(\left(\Omega_{1}, 0\right),\left(0, \frac{\Omega_{2}}{2}\right),\left(0, \frac{\Omega_{2}}{2}\right)\right)$. Following the argument in Saijo et al. (1999; Proof of Lemma 1), we can find a utility profile $u^{*}$ such that, for all participants $i, \Lambda_{i}^{N P}\left(\left(\left(\Omega_{1}, 0\right), x_{-i}\right), p\right) \subseteq L\left(z_{i}(p, x), u_{i}^{*}\right)$ but $z(p, x) \notin N P\left(u^{*}\right)$. Our Figure illustrates such a utility profile $u^{*}$, where $u_{2}^{*}$ and $u_{3}^{*}$ induce respectively the indifference curves $I_{2}^{*}$ and $I_{3}^{*}$ through the bundle $\left(0, \frac{\Omega_{2}}{2}\right)$, denoted by the line $A C$, while $u_{1}^{*}$ induces the indifference 
curve $I_{1}^{*}$ through the bundle $\left(\Omega_{1}, 0\right)$, represented by the lines $A D$ and $D E$. For the sake of simplicity, let $u^{*}$ represent a profile of homothetic preferences. It follows that the indifference curve $I_{1}^{*}$ of $u_{1}^{*}$ at $F \equiv\left(\frac{\Omega_{1}}{2}, \frac{\Omega_{2}}{4}\right)$ is represented by the lines $H F$ and $F G$. Note that each Pareto efficient allocation $x^{\prime} \in P\left(u^{*}\right)$ has the property that the bundle $x_{1}^{\prime}$ is always on the ray from the origin which passes through the point $D$. Then, $z(p, x) \notin N P\left(u^{*}\right)$ holds, as shown in Saijo et al. (1999; Proof of Lemma 1). Because $\mathcal{H}=2^{N} \backslash \varnothing$, let us consider the case that $H=\{2\}$. Since the premises of condition $\overline{G P Q}^{*}$ (iii) are fulfilled, this condition implies that $\left(x_{2}, \hat{x}_{-2}\right) \notin N P\left(u^{*}\right)$ for all $\hat{x}_{-2} \in \mathbb{R}^{2 \times 2}$. However, it can be easily checked that $\left(\hat{x}_{1}, x_{2}, \hat{x}_{3}\right) \in N P\left(u^{*}\right)$, where $\hat{x}_{1}=\hat{x}_{3}=\left(\frac{\Omega_{1}}{2}, \frac{\Omega_{2}}{4}\right)$, yielding a contradiction. We conclude that $N P$ does not satisfy condition $\overline{G P Q}^{*}$ (iii), as sought.

Lemma 7. Let $n \geq 3$ and suppose that Assumption 1 holds with $\mathcal{H}=2^{N} \backslash \varnothing$. Then, the efficient and egalitarian-equivalent correspondence, $E E$, defined on $U_{N}=\bar{U}$, does not satisfy condition $\overline{G M}^{*}$.

Proof. It directly follows from Corollary 4, since condition $\overline{G M}^{*}$ implies condition $\widehat{G M}^{*}$.

From the above lemmata, the last result of this section follows.

Corollary 8. Let $n \geq 3$ and suppose that Assumption 1 holds. Then, neither the Pareto correspondence, $P$, the no-envy and efficient correspondence, $N P$, nor the efficient and egalitarian-equivalent correspondence, $E E$, each of which is defined on $U_{N}=\bar{U}$, is partially honest implementable by any natural price-quantity mechanism.

All the results of this section are summarized in Table 1 displayed in Section 8.

\section{$7 \quad$ The case of two agents}

In this section, we investigate the two-agent case. Before turning to the details of our investigation, let us clarify some features on which our analysis is based. Firstly, in the two-person case, a natural price-quantity mechanism can be regarded as equivalent to a natural price-allocation mechanism, given that the difference of the aggregate endowment and the quantity announced by an agent can be interpreted as the consumption bundle of the other agent. Then, in what follows, we focus on natural price-allocation mechanisms, where the message space of each agent $i$ is $M_{i} \equiv \Delta \times A$. Secondly, and consequently, for each participant $i$, the set of truthful messages is that defined in (1). Finally, it is assumed that $F$ selects only interior allocations, ${ }^{21}$ that is, $F(u) \subseteq A \cap \mathbb{R}_{++}^{n \ell}$ for each $u \in U_{N}$.

In the conventional framework of natural implementation, when $n=2$, Saijo et al. (1996a) show that a punishment condition, combined with a stronger variant of Maskin monotonicity, is necessary and sufficient for implementation by a natural price-allocation mechanism. The punishment condition can be stated as follows.

Condition PA2. For any $\left(p, x, p^{\prime}, x^{\prime}\right) \in \Delta \times A \times \Delta \times A$, with $(p, x) \neq\left(p^{\prime}, x^{\prime}\right), F^{-1}(x, p) \neq \varnothing$ and $F^{-1}\left(x^{\prime}, p^{\prime}\right) \neq \varnothing$, there exists $z\left(p, x, p^{\prime}, x^{\prime}\right) \in A$ such that:

(i) $z_{1}\left(p, x, p^{\prime}, x^{\prime}\right) \in \Lambda_{1}^{F}(x, p)$ and $z_{2}\left(p, x, p^{\prime}, x^{\prime}\right) \in \Lambda_{2}^{F}\left(x^{\prime}, p^{\prime}\right)$;

(ii) for all $u^{*} \in U$, if $\Lambda_{1}^{F}(x, p) \subseteq L\left(z_{1}\left(p, x, p^{\prime}, x^{\prime}\right), u_{1}^{*}\right)$ and $\Lambda_{2}^{F}\left(x^{\prime}, p^{\prime}\right) \subseteq L\left(z_{2}\left(p, x, p^{\prime}, x^{\prime}\right), u_{2}^{*}\right)$, then $z\left(p, x, p^{\prime}, x^{\prime}\right) \in F\left(u^{*}\right)$.

\footnotetext{
${ }^{21}$ This is because otherwise, $F$ does not necessarily satisfy the no-veto power condition (Maskin, 1999); which makes the issue more complicated.
} 
In abstract social choice environments, Lombardi and Yoshihara (2011a) show that a punishment condition is indispensable for partially honest implementation in the two-person case. As a consequence, we amend the condition $P A 2$ as follows.

Weak Condition PA2 $(\overline{P A 2})$. There exists a map $z: \Delta \times A \times \Delta \times A \rightarrow Q^{2}$ such that, for any $H \in \mathcal{H}$ and any $\left(p, x, p^{\prime}, x^{\prime}\right) \in \Delta \times A \times \Delta \times A$ :

(i) the following requirements are satisfied:

(a) if $F^{-1}(x, p) \neq \varnothing$ and $F^{-1}\left(x^{\prime}, p^{\prime}\right) \neq \varnothing$, then $z\left(p, x, p^{\prime}, x^{\prime}\right) \in A$, with $z\left(p, x, p^{\prime}, x^{\prime}\right)=x$ if $(p, x)=\left(p^{\prime}, x^{\prime}\right)$, such that $z_{1}\left(p, x, p^{\prime}, x^{\prime}\right) \in \Lambda_{1}^{F}(x, p)$ and $z_{2}\left(p, x, p^{\prime}, x^{\prime}\right) \in \Lambda_{2}^{F}\left(x^{\prime}, p^{\prime}\right)$;

(b) if $F^{-1}(x, p) \neq \varnothing, F^{-1}\left(x^{\prime}, p^{\prime}\right)=\varnothing$, with $x^{\prime} \in F(u)$ for some $u \in U_{N}$, then $z_{1}\left(p, x, p^{\prime}, x^{\prime}\right) \in$ $\Lambda_{1}^{F}(x, p)$;

(c) if $F^{-1}(x, p)=\varnothing, F^{-1}\left(x^{\prime}, p^{\prime}\right) \neq \varnothing$, with $x \in F(u)$ for some $u \in U_{N}$, then $z_{2}\left(p, x, p^{\prime}, x^{\prime}\right) \in$ $\Lambda_{2}^{F}\left(x^{\prime}, p^{\prime}\right)$;

(d) otherwise, $z\left(p, x, p^{\prime}, x^{\prime}\right)=\mathbf{0}$;

(ii) for all $u \in U_{N}$, and all $\left(p, x, p^{\prime}, x^{\prime}\right) \in \Delta \times A \times \Delta \times A$, with $(p, x) \neq\left(p^{\prime}, x^{\prime}\right)$, if $F^{-1}(x, p) \neq$ $\varnothing$ and $F^{-1}\left(x^{\prime}, p^{\prime}\right) \neq \varnothing, \Lambda_{1}^{F}(x, p) \subseteq L\left(z_{1}\left(p, x, p^{\prime}, x^{\prime}\right), u_{1}\right), \Lambda_{2}^{F}\left(x^{\prime}, p^{\prime}\right) \subseteq L\left(z_{2}\left(p, x, p^{\prime}, x^{\prime}\right), u_{2}\right)$, and $z\left(p, x, p^{\prime}, x^{\prime}\right) \notin F(u)$, then there exists $h \in H$ such that:

(a) if $h=1$, then $x^{\prime} \notin F(u)$ and for some $\left(p^{\prime \prime}, x^{\prime \prime}\right) \in \Delta \times A$, with $x^{\prime \prime} \in F(u), z_{1}\left(p, x, p^{\prime \prime}, x^{\prime \prime}\right) \in$ $\partial L\left(z_{1}\left(p, x, p^{\prime}, x^{\prime}\right), u_{1}\right)$, or

(b) if $h=2$, then $x \notin F(u)$ and for some $\left(p^{\prime \prime}, x^{\prime \prime}\right) \in \Delta \times A$, with $x^{\prime \prime} \in F(u), z_{2}\left(p^{\prime \prime}, x^{\prime \prime}, p^{\prime}, x^{\prime}\right) \in$ $\partial L\left(z_{2}\left(p, x, p^{\prime}, x^{\prime}\right), u_{2}\right)$.

In the above condition, the requirements (b)-(c) imposed by condition $\overline{P A 2}(\mathrm{i})$ are satisfied by any $S C C$ because the mapping $z$ can be defined as $z_{1}\left(p, x, p^{\prime}, x^{\prime}\right) \equiv \mathbf{0} \in \Lambda_{1}^{F}(x, p)$ and $z_{2}\left(p, x, p^{\prime}, x^{\prime}\right) \equiv \mathbf{0} \in \Lambda_{2}^{F}\left(x^{\prime}, p^{\prime}\right)$. However, checking of the condition $\overline{P A 2}($ ii) can be a difficult matter because it may require a specific type of the mapping $z$.

In line with earlier results, the next theorem (whose proof is relegated to the Appendix) shows that even in two-agent economies the scope of implementation by natural priceallocation mechanisms is enlarged when some of the agents are partially honest, though limits still remain.

Theorem 4. Let $n=2$ and $\ell \geq 3$. Suppose that Assumption 1 holds. Let $F \in \mathcal{F}$ be an SCC such that $F(u) \subseteq A \cap \mathbb{R}_{++}^{n \ell}$ for all $u \in U_{N}$. Then, $F$ is partially honest implementable by a natural price-allocation mechanism if and only if it satisfies condition $\overline{P A 2}$.

While the above theorem fully identifies the class of $S C C$ s that are partially honest implementable by natural price-allocation mechanisms in two-agent economies endowed with more than two commodities, an analogous theorem can be given for two-agent and twocommodity economies for the sake of completeness. To this end, the definition of partially honest implementation by natural price-allocation mechanisms must be slightly modified to apply to these kinds of economies. Before introducing this definition, some additional notation is needed. Let $\bar{\Delta} \equiv\left\{p \in \mathbb{R}_{+}^{2} \mid \sum_{i=1}^{2} p_{i} \leq 1\right\}$ be the set of price announcements that each participant is allowed to report. Moreover, let $\varphi: \bar{\Delta} \rightarrow \Delta$ be a surjection as follows: for any $p \in \bar{\Delta}, \varphi(p) \in \Delta$ is such that $\frac{\varphi_{1}(p)}{\varphi_{2}(p)}=\frac{p_{1}}{p_{2}}$.

Definition 7. Let $\# N=2$ and $\ell=2$. An $S C C F \in \mathcal{F}$ is partially honest implementable by a natural price-allocation mechanism if there exists a mechanism $\gamma$ which satisfies DEFINITION 3-(i), (iv), (v), and:

(ii) for each $i \in N, M_{i}=\bar{\Delta} \times A$; and 
(iii) for each $u \in U_{N}$, each $x \in F(u)$, and each $\varphi(p) \in \Pi(x, u)$, if $m_{i}=(p, x)$ for each $i \in N$, then $m \in N E\left(\gamma, \succcurlyeq^{u}\right)$ and $g(m)=x$.

Based on this definition, condition $\overline{P A 2}$ can be shown to be necessary and sufficient for partially honest implementation by natural price-allocation mechanisms for two-agent and two-commodity economies (see Theorem 5 in the Appendix).

Finally, let us briefly discuss some implications of the results reported here. Firstly, because the constrained Walrasian correspondence satisfies condition $\overline{P A 2}$, which can be shown as in the proof of Lemma 3, this $S C C$ is partially honest implementable by a natural price-allocation mechanism even in two-agent economies. Secondly, as discussed in Saijo et al. (1996a), while the no-envy and efficient correspondence $N P$ satisfies condition $\overline{P A 2}$, making it partially honest implementable by a natural price-allocation mechanism in twoagent economies, the Pareto correspondence $P$ does not satisfy the assertion (a) of condition $\overline{P A 2}(\mathrm{i})$. The latter remark implies that the Pareto correspondence is not partially honest implementable by any natural price-allocation mechanism in two-agent economies. Finally, let $\bar{U}=U_{N}$ be the class of all profiles of utility functions which are continuous, quasiconcave, and strictly monotonic. The following results show that the efficient and egalitarianequivalent correspondence, $E E$, defined on $\bar{U}=U_{N}$, is partially honest implementable by natural price-allocation mechanisms even in two-agent economies.

Lemma 8. Let $n=2$. Then, the efficient and egalitarian-equivalent correspondence, $E E$, defined on $U_{N}=\bar{U}$, satisfies condition PA2.

Proof. See the Appendix.

Corollary 9. Let $n=2$ and suppose that Assumption 1 holds. Then, the efficient and egalitarian-equivalent correspondence, $E E$, defined on $U_{N}=\bar{U}$, is partially honest implementable by a natural price-allocation mechanism.

A summary of the results reported above can be found in Table 1 displayed in the next section.

\section{Concluding remarks}

This paper examined the problem of fully implementing efficient $S C C$ s in classical exchange economies by natural mechanisms when some of the participants are partially honest. The problem is formalized by considering only a minimal departure from the standard framework of natural implementation. First, it is postulated that the mechanism designer knows that there are partially honest agents involved in the mechanism, but not who these agents are. Second, the paper posited that partially honest agents have only "minimal" intrinsic preferences for honesty. The mechanism designer's implementation problem is to design a mechanism in such a way that, regardless of the current economy and the partially honest agents involved in the mechanism, only the $F$-optimal outcomes emerge as the equilibrium outcomes of the devised game form.

Several concepts of natural implementation are explored. New necessary and sufficient conditions for implementation by natural mechanisms are presented. On the basis of these conditions, while the class of $S C C$ s that are implementable by a price-quantity mechanism is significantly enlarged when there are partially honest agents, the scope of natural implementation is drastically enlarged when larger strategy spaces are considered. Remarkably, 
when there are more than two participants, any efficient $S C C$ is partially honest implementable by a natural price-quantity ${ }^{n-1}$ mechanism, and thus by a natural price-allocation mechanism. Surprisingly, the (unconstrained) Walrasian correspondence is partially honest implementable even via a natural price-quantity mechanism in two-commodity economies. The reported results stand in stark contrast with those of the standard literature on natural implementation theory. These results are summarized in Table 1.

TABLE 1

A SUMMARY OF THE REPORTED RESULTS

\begin{tabular}{|c|c|c|c|c|}
\hline \multirow{2}{*}{$\begin{array}{c}\text { No of Participants: } \\
\text { Mechanisms: }\end{array}$} & \multirow{2}{*}{$\begin{array}{c}n=2 \\
P Q=P A\end{array}$} & \multicolumn{3}{|c|}{$n \geq 3$} \\
\hline & & $P Q$ & $P Q^{2}$ & $P A=P Q^{n-1}$ \\
\hline $\operatorname{IrP}$ & yes & yes & & \\
\hline$N P$ & yes & no & yes & \\
\hline$E E$ & yes & no & no & yes \\
\hline$S C C s:$ & yes & yes & & \\
\hline$W: \ell=2$ & yes & yes & & \\
\hline$W: \ell \geq 3$ & yes & & & yes \\
\hline$P$ & no & no & no & yes \\
\hline
\end{tabular}

$P A$, price-allocation mechanism; $\operatorname{Ir} P$, the individual rational and efficient correspondence; $N P$, the no-envy and efficient correspondence; $E E$, the egalitarian-equivalent and efficient correspondence; $W_{c}$, the constrained Walrasian correspondence; $W$, the Walrasian correspondence; $P$, the Pareto correspondence.

\section{Appendix}

Proof of Theorem 1. Let $n \geq 3$ and Assumption 1 hold. Take any $F \in \mathcal{F}$. Let $\gamma \equiv(M, g)$ be a price-quantity ${ }^{n-1}$ mechanism. A message announced by agent $i \in N$ is denoted by $m_{i}=\left(p^{i}, x_{i}^{i}, x_{i+1}^{i}, \ldots, x_{i+n-2}^{i}\right) \in \Delta \times Q^{n-1}$, where $x_{i+k}^{i}$ denotes agent $i+k$ 's consumption bundle announced by agent $i$, for $k \in\{1, \ldots, n-2\}$. For each $i \in N$, the set of truthful messages is that defined in (2). Fix any triple $(m, p, x) \in M \times \Delta \times A$. The message profile $m$ is:

(i) consistent with $p$ and $x$ if, for all $j \in N, p^{j}=p$, and $x_{j}^{j-k}=x_{j}^{j}=x_{j}$ for $k \in\{1, \ldots, n-2\}$;

(ii) $m_{-i}$ consistent with $p$ and $x$ if, for all $j \in N \backslash\{i\}, p^{j}=p, x_{j}^{j-k}=x_{j}^{j}=x_{j}$ for $k \in$ $\{1, \ldots, n-2\}$, with $j-k \neq i, x_{i}^{i-k}=x_{i}$ for $k \in\{1, \ldots, n-2\}$, and $\left(p^{i}, x_{i}^{i}, x_{i+1}^{i}, \ldots, x_{i+n-2}^{i}\right) \neq$ $\left(p, x_{i}, x_{i+1}, \ldots, x_{i+n-2}\right)$;

where $\ell-k$ is regarded as $n-\ell^{*} \in N$ if $\ell-k=-\ell^{*}$ for $\ell \in\{j, i\}$.

The outcome function $g$ of $\gamma$ is defined as follows:

Rule 1: If $m$ is consistent with $p$ and $x$, where $p \in \Pi(x, u)$ and $x \in F(u)$ for some $u \in U_{N}$, then $g(m)=x$.

Rule 2: If $m$ is $m_{-i}$ consistent with $p$ and $x$, where $p \in \Pi(x, u)$ and $x \in F(u)$ for some $u \in U_{N}$, then $g(m)=x$.

Rule 3: Otherwise, $g_{i^{*}}(m)=\Omega$ and $g_{j}(m)=0$ for all $j \neq i^{*}$, where $i^{*}$ is defined as follows. Without loss of generality, let us suppose that $\Omega_{1} \geq 1$. Let $\sum_{i \in N} x_{i 1}^{i}=t$. Furthermore, by Definition $4(\mathrm{ii})$, it follows that $x_{i 1}^{i} \in\left[0, \Omega_{1}\right]$ for all $i \in N$. Let $v$ be an integer such that 
$v \leq t<v+1$. Therefore, $t=v+s$ where $s \in[0,1)$. It follows that there is a unique agent $i^{*} \in N$ such that $s \in\left[\frac{i^{*}-1}{n}, \frac{i^{*}}{n}\right)$.

According to the proposed construction, $\gamma$ is individually feasible and balanced; moreover, it satisfies forthrightness and the best response property.

We show that $F(u)=N A\left(\gamma, \succcurlyeq^{u}\right)$ holds for any $u \in U_{N}$ and any $H \in \mathcal{H}$. Take any $u \in U_{N}$ and any $H \in \mathcal{H}$.

First, let us show that $F(u) \subseteq N A\left(\gamma, \succcurlyeq^{u}\right)$ for this $H$. Let $x \in F(u)$, and assume that, for all $i \in N, m_{i}=\left(p^{i}, x_{i}^{i}, \ldots, x_{i+n-2}^{i}\right)=\left(p, x_{i}, \ldots, x_{i+n-2}\right) \in T_{i}^{\gamma}(u, F)$, where $p \in \Pi(x, u)$ and the tuple $\left(x_{i}, \ldots, x_{i+n-2}\right)$ is the $n-1$ tuple of $x$. From Rule 1 it follows that $g(m)=x$. Unilateral deviations from this announcement can only induce Rule 2. Therefore, by Rule 2, we have that, for all $i \in N, g\left(M_{i}, m_{-i}\right)=x$. It follows that $x \in N A\left(\gamma, \succcurlyeq^{u}\right)$ for this $H$.

Conversely, for the fixed $H$, let $m \in N E\left(\gamma, \succcurlyeq^{u}\right)$. Since one cannot have a Nash equilibrium message profile under Rules 2-3, it follows that $m$ falls into Rule 1. Then, $g(m)=x \in F\left(u^{\prime}\right)$ for some $u^{\prime} \in U_{N}$. Suppose that there is a partially honest agent $h \in N$ such that $m_{h} \notin T_{h}^{\gamma}(u, F)$. Then, she can induce Rule $\mathscr{2}$ by changing $m_{h}$ to $m_{h}^{\prime}=\left(p^{\prime}, x_{-(h+n-1)}^{\prime}\right) \in T_{h}^{\gamma}(u, F)$ and obtain $g_{h}\left(m_{h}^{\prime}, m_{-h}\right)=g_{h}\left(m_{h}, m_{-h}\right)=x_{h}$. Therefore, $\left(\left(m_{h}^{\prime}, m_{-h}\right), m\right) \in \succ_{h}^{u}$, which contradicts that $m \in N E\left(\gamma, \succcurlyeq^{u}\right)$ for the given $H$. Therefore, every partially honest agent $h$ is reporting truthfully. From the uniqueness of $x_{h+n-1}$, it follows that $x \in F(u)$, as sought.

As the above arguments hold for any $H \in \mathcal{H}$ and any $u \in U_{N}$, the statement follows.

Proof of Theorem 2. Let $n \geq 3$ and Assumption 1 hold. Take any $F \in \mathcal{F}$. Denote an arbitrary partially honest agent by $h \in N$. For each $i \in N$, the set of truthful messages is that defined in (3).

Suppose that $F$ is partially honest implementable by a natural price-quantity ${ }^{2}$ mechanism $\gamma$. We show that $F$ satisfies condition $\widehat{G M}^{*}$. Let $(H, u, x, p) \in \mathcal{H} \times U_{N} \times F(u) \times \pi^{F}(x, u)$ be given. For each $i \in N$, let $m_{i}=\left(p, x_{i}, x_{i+1}\right) \in \Delta \times Q^{2}$, where $n+1=1$. Then, for all $u^{\prime} \in F^{-1}(x, p), g(m)=x$ and $m \in N E\left(\gamma, \succcurlyeq^{u^{\prime}}\right)$ by forthrightness. Observe that $m \in$ $N E\left(\gamma, \succcurlyeq^{u^{\prime}}\right)$ for all $H^{\prime} \in \mathcal{H}$ and all $u^{\prime} \in F^{-1}(x, p)$. This implies that $g_{i}\left(M_{i}, m_{-i}\right) \subseteq L\left(x_{i}, u_{i}^{\prime}\right)$ for all $u^{\prime} \in F^{-1}(x, p)$. Then, $g_{i}\left(M_{i}, m_{-i}\right) \subseteq \Lambda_{i}^{F}(x, p)$ for all $i \in N$. Let $x \notin F\left(u^{*}\right)$ for some $u^{*} \in U_{N}$ and $\Lambda_{i}^{F}(x, p) \subseteq L\left(x_{i}, u_{i}^{*}\right)$ for all $i \in N$. Therefore, $g(m)=x \notin N A\left(\gamma, \succcurlyeq^{u^{*}}\right)$ for all $H^{\prime} \in \mathcal{H}$. We have that $\left(\left(m_{i}^{\prime}, m_{-i}\right), m\right) \in \succ_{i}^{u^{*}}$ for some $i \in N$ and some $m_{i}^{\prime} \in M_{i}$, which implies that $u_{i}^{*}\left(g_{i}\left(m_{i}^{\prime}, m_{-i}\right)\right) \geq u_{i}^{*}\left(g_{i}(m)\right)$. However, from $g_{i}\left(m_{i}^{\prime}, m_{-i}\right) \in g_{i}\left(M_{i}, m_{-i}\right) \subseteq L\left(x_{i}, u_{i}^{*}\right)$, it follows that $u_{i}^{*}\left(g_{i}\left(m_{i}^{\prime}, m_{-i}\right)\right)=u_{i}^{*}\left(g_{i}(m)\right)$. Thus, the deviator $i$ is a partially honest agent, that is, $i \in H$. Therefore, $m_{i} \notin T_{i}^{\gamma}\left(u^{*}, F\right)$ and $m_{i}^{\prime} \in T_{i}^{\gamma}\left(u^{*}, F\right)$, otherwise we fall into a contradiction. Then, $\left(x_{i}, x_{i+1}, x_{-\{i, i+1\}}^{\prime}\right) \notin F\left(u^{*}\right)$ for all $x_{-\{i, i+1\}}^{\prime} \in \mathbb{R}^{(n-2) \ell}$. Moreover, $\left(p, x_{i}^{*}, x_{i+1}^{*}\right) \in T_{i}^{\gamma}\left(u^{*}, F\right)$ and $\left(x_{i}^{*}, x_{i+1}^{*}\right) \neq\left(x_{i}, x_{i+1}\right)$ hold for each $x^{*} \in F\left(u^{*}\right)$, since otherwise, a contradiction is obtained. We conclude that, for some $i \in H, x_{i} \neq x_{i}^{*}$ or $x_{i+1} \neq x_{i+1}^{*}$ for all $x^{*} \in F\left(u^{*}\right)$, as sought. Since it holds for any $H \in \mathcal{H}$, we conclude that $F$ satisfies condition $\widehat{G M}^{*}$.

Next, we prove sufficiency. Suppose that $F$ satisfies condition $\widehat{G M}^{*}$. Consider the following price-quantity ${ }^{2}$ mechanism $\gamma \equiv(M, g)$. For each $i \in N$, let $M_{i} \equiv \Delta \times Q^{2}$. A message announced by agent $i \in N$ is denoted by $m_{i}=\left(p^{i}, x_{i}^{i}, x_{i+1}^{i}\right) \in \Delta \times Q^{2}$, where $x_{i+1}^{i}$ denotes agent $i+1$ 's consumption bundle announced by agent $i$. Take any $(m, p, x) \in M \times \Delta \times A$. The message profile $m$ is: 
(i) consistent with $p$ and $x$ if, for each $j \in N, p^{j}=p$ and $x_{j}^{j}=x_{j}^{j-1}=x_{j}$;

(ii) $m_{-i}$ consistent with $p$ and $x$, with $i \in N$, if (a) for each $j \in N \backslash\{i\}, p^{j}=p$, and (b) for each $j \in N \backslash\{i, i+1\}, x_{j}^{j}=x_{j}^{j-1}=x_{j}, x_{i}^{i-1}=x_{i}, x_{i+1}^{i+1}=x_{i+1}$, and $\left(p^{i}, x_{i}^{i}, x_{i+1}^{i}\right) \neq$ $\left(p, x_{i}, x_{i+1}\right)$;

where $1-1=n$ and $n+1=1$.

The outcome function $g$ of $\gamma$ is defined as follows:

Rule 1: If $m$ is consistent with $p$ and $x$, where $x \in F(u)$ and $p \in \pi^{F}(x, u)$ for some $u \in U_{N}$, then $g(m)=x$.

Rule 2: If there exists a unique $i \in N$ such that $m$ is $m_{-i}$ consistent with $p$ and $x$, where $x \in F(u)$ and $p \in \pi^{F}(x, u)$ for some $u \in U_{N}$, and $\left(p^{i}, x_{i}^{i}, x_{i+1}^{i}\right) \neq\left(p, x_{i}, x_{i+1}\right)$, then:

Rule 2.1: if $p^{i} \neq p$, then $g(m)=x$;

Rule 2.2: if $p^{i}=p$ and there exists $\bar{x}_{-\{i, i+1\}} \in \mathbb{R}^{(n-2) \ell}$ such that $\left(\bar{x}_{-\{i, i+1\}}, x_{i}^{i}, x_{i+1}^{i}\right) \in F\left(u^{\prime}\right)$ for some $u^{\prime} \in U_{N}$, then $g(m)=x$;

Rule 2.3: otherwise,

$$
g(m)= \begin{cases}\left(x_{i}^{i},\left(\frac{\Omega-x_{i}^{i}}{n-1}\right)_{j \neq i}\right) & \text { if } x_{i}^{i} \in \Lambda_{i}^{F}(x, p) \\ x & \text { otherwise }\end{cases}
$$

Rule 3: Otherwise, $g_{i^{*}}(m)=\Omega$ and $g_{j}(m)=0$ for all $j \neq i^{*}$, where $i^{*}$ is defined as follows. Without loss of generality, let us suppose that $\Omega_{1} \geq 1$. Let $\sum_{i \in N} x_{i 1}^{i}=t$. Furthermore, by Definition $5\left(\right.$ ii), it follows that $x_{i 1}^{i} \in\left[0, \Omega_{1}\right]$ for all $i \in N$. Let $v$ be an integer such that $v \leq t<v+1$. Therefore, $t=v+s$ where $s \in[0,1)$. It follows that there is a unique agent $i^{*} \in N$ such that $s \in\left[\frac{i^{*}-1}{n}, \frac{i^{*}}{n}\right)$.

According to the proposed construction, $\gamma$ is individually feasible and balanced; moreover, it satisfies forthrightness and the best response property.

We show that $F(u)=N A\left(\gamma, \succcurlyeq^{u}\right)$ holds for any $u \in U_{N}$ and any $H \in \mathcal{H}$. Take any $u \in U_{N}$ and any $H \in \mathcal{H}$.

First, we show $F(u) \subseteq N A\left(\gamma, \succcurlyeq^{u}\right)$. Let $x \in F(u)$ and $p \in \pi^{F}(x, u)$. Let $m_{i}=$ $\left(p, x_{i}, x_{i+1}\right) \in T_{i}^{\gamma}(u, F)$ for each $i \in N$. Then, by Rule $1, g(m)=x$. Suppose that agent $i \in N$ deviates from $m_{i}$ to a different message $m_{i}^{\prime} \in M_{i}$. By the definition of $g$, we have that any deviation of agent $i$ will get her to an outcome in $\Lambda_{i}^{F}(x, p)$, so that $g_{i}\left(M_{i}, m_{-i}\right)=$ $\Lambda_{i}^{F}(x, p) \subseteq L\left(x_{i}, u_{i}\right)$. Since $m_{i} \in T_{i}^{\gamma}(u, F)$ for any $i \in N$, it follows that $x \in N A\left(\gamma, \succcurlyeq^{u}\right)$ for this $H$, as sought.

Conversely, for this $H$, we show $N A\left(\gamma, \succcurlyeq^{u}\right) \subseteq F(u)$. Let $m \in N E\left(\gamma, \succcurlyeq^{u}\right)$ for this $H$. Because $m$ cannot correspond to Rules 2-3, $m$ falls into Rule 1. Thus, there exists a $p \in \Delta$ and an $x \in A$, with which $m$ is consistent with, such that $x \in F\left(u^{\prime}\right)$ and $p \in \pi^{F}\left(x, u^{\prime}\right)$ for some $u^{\prime} \in U_{N}$. Then, $g(m)=x$. By Rule $2, \Lambda_{i}^{F}(x, p)=g_{i}\left(M_{i}, m_{-i}\right) \subseteq L\left(x_{i}, u_{i}\right)$ for all $i \in N$. We show that $m_{h} \in T_{h}^{\gamma}(u, F)$ for every $h \in H$. Assume, to the contrary, that $m_{h} \notin T_{h}^{\gamma}(u, F)$ for some $h \in H$. Agent $h$ can change $m_{h}$ to $m_{h}^{\prime}=\left(p, x_{h}^{h}, x_{h+1}^{h}\right) \in T_{h}^{\gamma}(u, F)$. If $x_{h}^{h} \neq x_{h}$ and $x_{h+1}^{h} \neq x_{h+1}$, then it is obvious that agent $h$ is the unique deviator and Rule 2 applies. Otherwise, let $x_{h}^{h} \neq x_{h}$ and $x_{h+1}^{h}=x_{h+1}$. Since $x \in A$ and $\left(x_{-h}, x_{h}^{h}\right) \notin A$, it follows that $h$ is the unique deviator and Rule 2 applies again. A similar argument applies to the case in which $x_{h}^{h}=x_{h}$ and $x_{h+1}^{h} \neq x_{h+1}$. Since $m_{h}^{\prime} \in T_{h}^{\gamma}(u, F)$, it follows that there exists $\bar{x}_{-\{h, h+1\}} \in \mathbb{R}^{(n-2) \ell}$ such that $\left(\bar{x}_{-\{h, h+1\}}, x_{h}^{h}, x_{h+1}^{h}\right) \in F(u)$, so that $g_{h}\left(m_{h}^{\prime}, m_{-h}\right)=x_{h}$ by Rule 2.2. We have that $\left(\left(m_{h}^{\prime}, m_{-h}\right), m\right) \in \succ_{h}^{u}$ for this $h \in H$, which contradicts that 
$m \in N E\left(\gamma, \succcurlyeq^{u}\right)$ for the given $H$. Therefore, $m_{h}=\left(p, x_{h}, x_{h+1}\right) \in T_{h}^{\gamma}(u, F)$ for all $h \in H$. Thus, for the given $H$, condition $\widehat{G M}^{*}$ implies that $x \in F(u)$.

As the above arguments hold for any $H \in \mathcal{H}$ and any $u \in U_{N}$, the statement follows.

Proof of Theorem 3. Let $n \geq 3$ and suppose that Assumption 1 holds. Take any $F \in \mathcal{F}$. For each $i \in N$, the set of truthful messages is that defined in (4).

Let us first show the necessity of condition $\overline{G M}^{*}$ and condition $\overline{G P Q}^{*}$. To that purpose, suppose that $F$ is partially honest implementable by a natural price-quantity mechanism.

First, we show that $F$ meets condition $\overline{G M}^{*}$. Let $(H, u, x, p) \in \mathcal{H} \times U_{N} \times F(u) \times \pi^{F}(x, u)$ be given. For each $i \in N$, let $m_{i} \equiv\left(p, x_{i}\right) \in \Delta \times Q$. Then, for any $u^{\prime} \in F^{-1}(x, p)$, $g(m)=x$ and $m \in N E\left(\gamma, \succcurlyeq^{u^{\prime}}\right)$ hold by forthrightness. Thus, $g_{i}\left(M_{i}, m_{-i}\right) \subseteq L\left(x_{i}, u_{i}^{\prime}\right)$. Since it holds for any $u^{\prime} \in F^{-1}(x, p)$, it follows that $g_{i}\left(M_{i}, m_{-i}\right) \subseteq \Lambda_{i}^{F}(x, p)$. Therefore, $g_{i}\left(M_{i}, m_{-i}\right) \subseteq \Lambda_{i}^{F}(x, p)$ for all $i \in N$. Observe that $m \in N E\left(\gamma, \succcurlyeq^{\prime}\right)$ for all $H^{\prime} \in \mathcal{H}$, and, for all $u^{\prime} \in F^{-1}(x, p), m_{i} \in T_{i}^{\gamma}\left(u^{\prime}, F\right)$ for all $i \in N$. Let $x \notin F\left(u^{*}\right)$ for some $u^{*} \in U_{N}$ and $\Lambda_{i}^{F}(x, p) \subseteq L\left(x_{i}, u_{i}^{*}\right)$ for all $i \in N$. Therefore, $g(m)=x \notin N E\left(\gamma, \succcurlyeq^{u^{*}}\right)$ for all $H^{\prime} \in \mathcal{H}$. Moreover, $\left(\left(m_{i}^{\prime}, m_{-i}\right), m\right) \in \succ_{i}^{u^{*}}$ for some $i \in N$ and some $m_{i}^{\prime} \in M_{i}$, which implies that $u_{i}^{*}\left(g_{i}\left(m_{i}^{\prime}, m_{-i}\right)\right) \geq u_{i}^{*}\left(g_{i}(m)\right)$. However, from $g_{i}\left(m_{i}^{\prime}, m_{-i}\right) \in g_{i}\left(M_{i}, m_{-i}\right) \subseteq L\left(x_{i}, u_{i}^{*}\right)$, it follows that $u_{i}^{*}\left(g_{i}\left(m_{i}^{\prime}, m_{-i}\right)\right)=u_{i}^{*}\left(g_{i}(m)\right)$. Thus, agent $i$ is a partially honest agent, that is, $i \in H$. It follows that $m_{i} \notin T_{i}^{\gamma}\left(u^{*}, F\right)$ and $m_{i}^{\prime} \equiv\left(p, x_{i}^{*}\right) \in T_{i}^{\gamma}\left(u^{*}, F\right)$, with $x_{i}^{*} \neq x_{i}$, otherwise we fall into a contradiction. We conclude that $x_{h} \neq x_{h}^{*}$ for all $x^{*} \in F\left(u^{*}\right)$ for some $h(=i) \in H$, as sought. Since it holds for any $H \in \mathcal{H}$, we conclude that $F$ satisfies condition $\overline{G M}^{*}$.

Next, we show that $F$ satisfies condition $\overline{G P Q}^{*}$. Take any $H \in \mathcal{H}$. Let $(p, x) \in \Delta \times Q^{n}$ be such that $I^{F}(p, x)=N$ and $x \notin A$. Take any $i \in N$ and any $u \in F^{-1}\left(\left(\Omega-\sum_{l \neq i} x_{l}, x_{-i}\right), p\right)$. Define $m_{l} \equiv\left(p, x_{l}\right)$ for all $l \in N \backslash\{i\}$ and $m_{i} \equiv\left(p, \Omega-\sum_{l \neq i} x_{l}\right)$. Forthrightness implies that $g(m)=\left(\Omega-\sum_{l \neq i} x_{l}, x_{-i}\right)$ and $m \in N E\left(\gamma, \succcurlyeq^{u}\right)$ for the given $H$. Because $m_{j} \in T_{j}^{\gamma}(u, F)$ for all $j \in N$, we have that $m \in N E\left(\gamma, \succcurlyeq^{u}\right)$ for each $H^{\prime} \in \mathcal{H}$. Moreover, we have that $g_{i}\left(M_{i}, m_{-i}\right) \subseteq L\left(\Omega-\sum_{l \neq i} x_{l}, u_{i}\right)$. Since the previous arguments hold for any $u \in F^{-1}\left(\left(\Omega-\sum_{l \neq i} x_{l}, x_{-i}\right), p\right)$ and any $i \in N$, it follows that $g_{i}\left(M_{i}, m_{-i}\right) \subseteq$ $\Lambda_{i}^{F}\left(\left(\Omega-\sum_{l \neq i} x_{l}, x_{-i}\right), p\right)$ for each $i \in N$. Let $z(p, x) \equiv g(\bar{m})$, where $\bar{m} \equiv\left(\left(p, x_{i}\right)_{i \in N}\right)$. Then, $z_{i}(p, x) \in \Lambda_{i}^{F}\left(\left(\Omega-\sum_{l \neq i} x_{l}, x_{-i}\right), p\right)$ for all $i \in N$. This proves condition $\overline{G P Q}^{*}(\mathrm{i})$.

For each $i \in N$, let us define the map $S_{i}\left(\cdot ;\left(p, x_{-i}\right)\right): \Delta \times Q \rightarrow Q$ as follows. For any $\left(p^{\prime}, x_{i}^{\prime}\right) \in \Delta \times Q$ :

(i) if there exist $u^{\prime} \in U_{N}$ and $x_{i}^{*} \in Q$ such that $I^{F}\left(p, x^{*}\right)=N, \Lambda_{j}^{F}\left(\left(\Omega-\sum_{l \neq j} x_{l}^{*}, x_{-j}^{*}\right), p\right) \subseteq$ $L\left(z_{j}\left(p, x^{*}\right), u_{j}^{\prime}\right)$ for all $j \in N$, where $x^{*} \equiv\left(x_{i}^{*}, x_{-i}\right)$, and $\left(x_{i}^{\prime}, \hat{x}_{-i}\right) \in F\left(u^{\prime}\right)$ for some $\hat{x}_{-i} \in$ $\mathbb{R}^{(n-1) \ell}$, then $S_{i}\left(\left(p^{\prime}, x_{i}^{\prime}\right) ;\left(p, x_{-i}\right)\right) \equiv g_{i}\left(\left(p^{\prime}, x_{i}^{\prime}\right), \bar{m}_{-i}\right)$;

(ii) otherwise, $S_{i}\left(\left(p^{\prime}, x_{i}^{\prime}\right) ;\left(p, x_{-i}\right)\right) \equiv \mathbf{0}$.

It is clear that $S_{i}\left(\left(p^{\prime}, x_{i}^{\prime}\right) ;\left(p, x_{-i}\right)\right) \in \Lambda_{i}^{F}\left(\left(\Omega-\sum_{l \neq i} x_{l}, x_{-i}\right), p\right)$ because $g_{i}\left(M_{i}, \bar{m}_{-i}\right) \subseteq$ $\Lambda_{i}^{F}\left(\left(\Omega-\sum_{l \neq i} x_{l}, x_{-i}\right), p\right)$ and $\mathbf{0} \in \Lambda_{i}^{F}\left(\left(\Omega-\sum_{l \neq i} x_{l}, x_{-i}\right), p\right)$. Thus, condition $\overline{G P Q}^{*}$ (ii) holds.

To show that condition $\overline{G P Q}^{*}$ (iii) is also satisfied, take any $u^{*} \in U_{N}$ and suppose that $\Lambda_{i}^{F}\left(\left(\Omega-\sum_{l \neq i} x_{l}, x_{-i}\right), p\right) \subseteq L\left(z_{i}(p, x), u_{i}^{*}\right)$ holds for all $i \in N$. Moreover, let $z(p, x) \equiv$ 
$g(\bar{m}) \notin F\left(u^{*}\right)=N A(\gamma, \succcurlyeq)$ for all $H^{\prime} \in \mathcal{H}$. Then, $\left(\left(m_{i}^{\prime}, \bar{m}_{-i}\right), \bar{m}\right) \in \succ_{i}^{u^{*}}$ for some $i \in N$ and some $m_{i}^{\prime} \in M_{i}$, which implies that $u_{i}^{*}\left(g_{i}\left(m_{i}^{\prime}, \bar{m}_{-i}\right)\right) \geq u_{i}^{*}\left(g_{i}(\bar{m})\right)$. Because $g_{i}\left(m_{i}^{\prime}, \bar{m}_{-i}\right) \in$ $g_{i}\left(M_{i}, \bar{m}_{-i}\right) \subseteq L\left(z_{i}(p, x), u_{i}^{*}\right), u_{i}^{*}\left(g_{i}\left(m_{i}^{\prime}, \bar{m}_{-i}\right)\right)=u_{i}^{*}\left(g_{i}(\bar{m})\right)$. Then, the deviator $i$ is a partially honest agent, that is, $i \in H$. Therefore, $m_{i}^{\prime} \equiv\left(p^{\prime}, x_{i}^{\prime}\right) \in T_{i}^{\gamma}\left(u^{*}, F\right)$ and $\bar{m}_{i} \notin T_{i}^{\gamma}\left(u^{*}, F\right)$, otherwise we fall into a contradiction. Let $h \equiv i$. Since $\bar{m}_{h} \notin T_{h}^{\gamma}\left(u^{*}, F\right)$, it follows that $\left(x_{h}, \hat{x}_{-h}\right) \notin F\left(u^{*}\right)$ for all $\hat{x}_{-h} \in \mathbb{R}^{(n-1) \ell}$. Thus, the assertion (a) of condition $\overline{G P Q}^{*}$ (iii) holds. Hence, we are left to show that the assertion (b) of this condition is verified by $F$ too. From the previous arguments, it follows that $g_{h}\left(m_{h}^{\prime}, \bar{m}_{-h}\right) \in \partial \Lambda_{h}^{F}\left(\left(\Omega-\sum_{l \neq h} x_{l}, x_{-h}\right), p\right) \cap$ $\partial L\left(z_{h}(p, x), u_{h}^{*}\right)$. Since $m_{h}^{\prime} \equiv\left(p^{\prime}, x_{h}^{\prime}\right) \in T_{h}^{\gamma}\left(u^{*}, F\right),\left(x_{h}^{\prime}, \hat{x}_{-h}\right) \in F\left(u^{*}\right)$ for some $\hat{x}_{-h} \in$ $\mathbb{R}^{(n-1) \ell}$. Thus, by the definition of the mapping $S_{h}\left(\cdot ;\left(p, x_{-h}\right)\right)$ in condition $\overline{G P Q}^{*}(\mathrm{ii})$, we have that $S_{h}\left(\left(p^{\prime}, x_{h}^{\prime}\right) ;\left(p, x_{-h}\right)\right) \equiv g_{h}\left(\left(p^{\prime}, x_{h}^{\prime}\right), \bar{m}_{-h}\right)$. Hence, $S_{h}\left(\left(p^{\prime}, x_{h}^{\prime}\right) ;\left(p, x_{-h}\right)\right) \in$ $\partial L\left(z_{h}(p, x), u_{h}^{*}\right)$, as sought. In summary, condition $\overline{G P Q}^{*}$ (iii) is satisfied.

Next, we prove sufficiency. Suppose that $F$ satisfies condition $\overline{G M}^{*}$ and condition $\overline{G P Q}^{*}$. Because of condition $\overline{G P Q}^{*}(\mathrm{ii})$, for each $i \in N$, there exists a map $S_{i}\left(\cdot ;\left(p, x_{-i}\right)\right): \Delta \times$ $Q \rightarrow Q$ which satisfies the requirements of condition $\overline{G P Q}^{*}$ (ii). For any vertex $\bar{p} \in \Delta$ and any other price vector $p \in \Delta$, let $\mathcal{B}_{\epsilon}(\bar{p} ; p)$ be a closed ball with center $\bar{p}$ and radius $\epsilon>0$ such that $\mathcal{B}_{\epsilon}(\bar{p} ; p) \subset \Delta$ and $p \notin \mathcal{B}_{\epsilon}(\bar{p} ; p)$. Since $\mathcal{B}_{\epsilon}(\bar{p} ; p)$ and $\Delta$ are homeomorphic, there is a bijection between $\phi: \mathcal{B}_{\epsilon}(\bar{p} ; p) \rightarrow \Delta$. Then, given $\left(p, x_{-i}\right) \in \Delta \times Q^{n-1}$ and a vertex $\bar{p} \in \Delta$, let us define, for each $i \in N$, the map $\widetilde{S}_{i}\left(\cdot ;\left(p, x_{-i}\right)\right): \mathcal{B}_{\epsilon}(\bar{p} ; p) \times Q \rightarrow Q$ as $\tilde{S}_{i}\left(\left(\hat{p}, \hat{x}_{i}\right) ;\left(p, x_{-i}\right)\right) \equiv S_{i}\left(\left(\phi(\hat{p}), \hat{x}_{i}\right) ;\left(p, x_{-i}\right)\right)$ for each $\left(\hat{p}, \hat{x}_{i}\right) \in \mathcal{B}_{\epsilon}(\bar{p} ; p) \times Q$. For any $x \in Q^{n}$, let $B\left(\Lambda_{i}^{F}(x, p)\right)$ be the upper boundary of $\Lambda_{i}^{F}(x, p)$, that is, $B\left(\Lambda_{i}^{F}(x, p)\right) \equiv$ $\left\{y_{i} \in Q \mid y_{i} \in \Lambda_{i}^{F}(x, p)\right.$ and $\nexists z_{i} \in \Lambda_{i}^{F}(x, p)$ such that $\left.z_{i} \gg y_{i}\right\}$.

Consider the following price-quantity mechanism $\gamma \equiv(M, g)$. For each $i \in N$, let $M_{i} \equiv$ $\Delta \times Q$. A message announced by agent $i \in N$ is denoted by $m_{i}=\left(p^{i}, x_{i}^{i}\right) \in \Delta \times Q$. Define its outcome function as follows:

Rule 1: If $m_{i}=\left(p, x_{i}\right)$ for all $i \in N$ such that $x \in F\left(u^{\prime}\right)$ and $p \in \pi^{F}\left(x, u^{\prime}\right)$ for some $u^{\prime} \in U_{N}$, then $g(m)=x$.

Rule 2: If $m_{i}=\left(p, x_{i}\right)$ for all $i \in N$ such that $x \notin A$ and $I^{F}(p, x)=N$, then $g(m)=z(p, x)$, where $z(p, x)$ is the allocation specified in condition $\overline{G P Q}^{*}(\mathrm{i})$.

Rule 3: If $m_{i}=\left(p, x_{i}\right)$ for all $i \in N, 1 \leq \# I^{F}(p, x) \leq n-1$, then

$$
g_{i}(m)=\left\{\begin{array}{ccc}
\frac{\Omega}{n-\left|I^{F}(p, x)\right|} & \text { if } & i \notin I^{F}(p, x) \\
\mathbf{0} & & \text { otherwise. }
\end{array}\right.
$$

Rule 4: If, for some $i \in N,\left(p, x_{l}\right)$ for all $l \in N \backslash\{i\}$, and $\left(p^{i}, x_{i}\right)$, with $p \neq p^{i}$, and $i \in I^{F}(p, x)$, then, take two distinct vertices $\bar{p}, \bar{p}^{\prime} \in \partial \Delta$, and:

Rule 4.1: if $p^{i}=\bar{p}^{\prime}$, then $g(m)=\left(\Omega-\sum_{l \neq i} x_{l}, x_{-i}\right)$;

Rule 4.2: if $p^{i} \in \mathcal{B}_{\epsilon}(\bar{p} ; p)$, then

$$
g(m)=\left\{\begin{array}{cc}
\left(\tilde{S}_{i}\left(\left(p^{i}, x_{i}\right) ;\left(p, x_{-i}\right)\right),\left(\frac{\Omega-\tilde{S}_{i}\left(\left(p^{i}, x_{i}\right) ;\left(p, x_{-i}\right)\right)}{n-1}\right)_{l \neq i}\right) & \text { if } \\
\left(x_{i},\left(\frac{\Omega-x_{i}}{n-1}\right)_{l \neq i}\right) & \text { if }\left\{\begin{array}{c}
\left.\left.\tilde{S}_{i}\left(\left(p^{i}, x_{i}\right) ;\left(p, x_{-i}\right)\right) \neq \mathbf{0}, x_{i}\right) ;\left(p, x_{-i}\right)\right)=\mathbf{0} \text { and } \\
x_{i} \in \Lambda_{i}^{F}\left(\left(\Omega-\sum_{l \neq i} x_{l}, x_{-i}\right), p\right)
\end{array}\right\} \\
\left(\psi_{i}\left(x_{i}\right),\left(\frac{\Omega-\psi_{i}\left(x_{i}\right)}{n-1}\right)_{l \neq i}\right) & \text { otherwise }
\end{array}\right.
$$


where $\psi_{i}: Q \backslash \Lambda_{i}^{F}\left(\left(\Omega-\sum_{l \neq i} x_{l}, x_{-i}\right), p\right) \rightarrow \partial \Lambda_{i}^{F}\left(\left(\Omega-\sum_{l \neq i} x_{l}, x_{-i}\right), p\right)$ is a surjective function;

Rule 4.3: otherwise, $g(m)=\left(\hat{x}_{i},\left(\frac{\Omega-\hat{x}_{i}}{n-1}\right)_{l \neq i}\right)$, where $\left\{\hat{x}_{i}\right\} \equiv B\left(\Lambda_{i}^{F}\left(\left(\Omega-\sum_{l \neq i} x_{l}, x_{-i}\right), p\right)\right) \cap$ $\left\{y_{i} \in \mathbb{R}_{+}^{\ell} \mid\right.$ there exists $\alpha \in \mathbb{R}_{+}$such that $\left.y_{i}=\alpha x_{i}\right\}$;

Rule 5: Otherwise, $g_{i^{*}}(m)=\Omega$ and $g_{j}(m)=0$ for all $j \neq i^{*}$, where $i^{*}$ is defined as follows. Without loss of generality, let us suppose that $\Omega_{1} \geq 1$. Let $\sum_{i \in N} y_{i 1}^{i}=t$. Furthermore, by Definition 6(ii), it follows that, for all $i \in N, y_{i 1} \in\left[0, \Omega_{1}\right]$. Let $v$ be an integer such that $v \leq t<v+1$. Therefore, $t=v+s$ where $s \in[0,1)$. It follows that there is a unique agent $i^{*} \in N$ such that $s \in\left[\frac{i^{*}-1}{n}, \frac{i^{*}}{n}\right)$.

According to the proposed construction, $\gamma$ is individually feasible and balanced; moreover, it satisfies forthrightness and the best response property.

We show that $F(u)=N A\left(\gamma, \succcurlyeq^{u}\right)$ holds for any $u \in U_{N}$ and any $H \in \mathcal{H}$. To this end, take any $u \in U_{N}$ and any $H \in \mathcal{H}$.

First, we show $F(u) \subseteq N A\left(\gamma, \succcurlyeq^{u}\right)$ for this $H$. Let $x \in F(u)$ and $p \in \pi^{F}(x, u)$. Let $m_{i}=\left(p, x_{i}\right) \in T_{i}^{\gamma}(u, F)$ for all $i \in N$. Rule 1 implies $g(m)=x$. Suppose that agent $i$ deviates from $m_{i}$ to a different message $m_{i}^{\prime} \in M_{i}$. By the definition of $g$, any deviation of agent $i \in N$ will get her to an outcome in $\Lambda_{i}^{F}(x, p)$ so that $g_{i}\left(M_{i}, m_{-i}\right) \subseteq \Lambda_{i}^{F}(x, p)$. Since $\Lambda_{i}^{F}(x, p) \subseteq L\left(x_{i}, u_{i}\right)$ for all $i \in N$ and every agent is truthful, it follows that $x \in N A\left(\gamma, \succcurlyeq^{u}\right)$ for this $H$.

Conversely, for the given $H \in \mathcal{H}$, we show $N A\left(\gamma, \succcurlyeq^{u}\right) \subseteq F(u)$. Let $m \in N E\left(\gamma, \succcurlyeq^{u}\right)$. Since $m$ cannot correspond to Rules 3-5, $m$ falls either into Rule 1 or into Rule 2.

Suppose that $m$ falls into Rule 1. Then, $m_{i}=\left(p, x_{i}\right)$ for all $i \in N$ such that $g(m)=$ $x \in F\left(u^{\prime}\right)$ and $p \in \pi^{F}\left(x, u^{\prime}\right)$ for some $u^{\prime} \in U_{N}$. By Rule 4.3, it follows that $\partial \Lambda_{i}^{F}(x, p) \subseteq$ $g_{i}\left(M_{i}, m_{-i}\right)$ for all $i \in N$, which further implies $\Lambda_{i}^{F}(x, p) \subseteq L\left(x_{i}, u_{i}\right)$ for all $i \in N$ given that $m \in N E\left(\gamma, \succcurlyeq^{u}\right)$ and our suppositions of agents' preferences. Take any $h \in H$ and suppose that $m_{h} \notin T_{h}^{\gamma}(u, F)$. Then, $\left(x_{h}, \bar{x}_{-h}\right) \notin F(u)$ for all $\bar{x}_{-h} \in \mathbb{R}^{(n-1) \ell}$. Take any $m_{h}^{\prime}=\left(p^{\prime}, x_{h}^{\prime}\right) \in$ $T_{h}^{\gamma}(u, F)$ with $p^{\prime}=\bar{p}^{\prime} \in \Delta \backslash\left\{\mathcal{B}_{\epsilon}(\bar{p} ; p) \cup\{p\}\right\}$. Rule 4.1 implies that $g_{h}\left(m_{h}^{\prime}, m_{-h}\right)=x_{h}$. So, $\left(m_{h}^{\prime}, m_{-h}\right) \succ_{h}^{u} m$, which contradicts that $m \in N E\left(\gamma, \succcurlyeq^{u}\right)$ for the given $H \in \mathcal{H}$. It follows that $m_{h} \in T_{h}^{\gamma}(u, F)$ for all $h \in H$. Therefore, for the given $H \in \mathcal{H}$, condition $\overline{G M}^{*}$ implies that $x \in F(u)$, as sought.

Suppose that $m$ falls into Rule 2. Then, $m_{i}=\left(p, x_{i}\right)$ for all $i \in N, I^{F}(p, x)=$ $N$, and $x \notin A$. This rule implies that $g(m)=z(p, x)$. By Rule 4.3, it follows that $\partial \Lambda_{i}^{F}\left(\left(\Omega-\sum_{l \neq i} x_{l}, x_{-i}\right), p\right) \subseteq g_{i}\left(M_{i}, m_{-i}\right)$ for all $i \in N$. Given that $m \in N E\left(\gamma, \succcurlyeq^{u}\right)$ and our suppositions of agents' preferences, it follows that $\Lambda_{i}^{F}\left(\left(\Omega-\sum_{l \neq i} x_{l}, x_{-i}\right), p\right) \subseteq$ $L\left(z_{i}(p, x), u_{i}\right)$ for all $i \in N$. By the supposition that $m \in N E\left(\gamma, \succcurlyeq^{u}\right)$, it follows that for any $h \in H, g_{h}\left(m_{h}^{\prime}, m_{-h}\right) \notin \partial L\left(z_{h}(p, x), u_{h}\right)$ if $m_{h} \notin T_{h}^{\gamma}(u, F)$ and $m_{h}^{\prime} \in T_{h}^{\gamma}(u, F)$, otherwise we fall into a contradiction. Suppose that $m_{h} \in T_{h}^{\gamma}(u, F)$ for all $h \in H$. Condition $\overline{G P Q}^{*}$ (iii) implies that $z(p, x) \in F(u)$, as sought. Otherwise, suppose that $m_{h} \notin T_{h}^{\gamma}(u, F)$ for some $h \in H$, so that $\left(x_{h}, \hat{x}_{-h}\right) \notin F(u)$ for all $\hat{x}_{-h} \in \mathbb{R}^{(n-1) \ell}$. Define $H^{\prime} \equiv\left\{h \in H \mid m_{h} \notin T_{h}^{\gamma}(u, F)\right\}$. Take any $h \in H^{\prime}$. It follows that for any $m_{h}^{\prime}=\left(p^{\prime}, x_{h}^{\prime}\right) \in$ $T_{h}^{\gamma}(u, F)$, with $p^{\prime} \in \mathcal{B}_{\epsilon}(\bar{p} ; p), g_{h}\left(m_{h}^{\prime}, m_{-h}\right) \notin \partial L\left(z_{h}(p, x), u_{h}\right)$. So, for each $h \in H^{\prime}$, it follows that $\tilde{S}_{h}\left(\left(p^{\prime}, x_{h}^{\prime}\right) ;\left(p, x_{-h}\right)\right) \notin \partial L\left(z_{h}(p, x), u_{h}\right)$ for all $\left(p^{\prime}, x_{h}^{\prime}\right) \in \mathcal{B}_{\epsilon}(\bar{p} ; p) \times Q$, with $\left(x_{h}^{\prime}, \hat{x}_{-h}\right) \in F(u)$ for some $\hat{x}_{-h} \in \mathbb{R}^{(n-1) \ell}$. Therefore, for all $\left(p^{\prime \prime}, x_{h}^{\prime}\right) \in \Delta \times Q$, where $\phi\left(p^{\prime}\right)=p^{\prime \prime}, S_{h}\left(\left(p^{\prime \prime}, x_{h}^{\prime}\right) ;\left(p, x_{-h}\right)\right) \notin \partial L\left(z_{h}(p, x), u_{h}\right)$. Moreover, if $H \backslash H^{\prime}$ is not an empty set, then $m_{h} \in T_{h}^{\gamma}(u, F)$ for $h \in H \backslash H^{\prime}$. Condition $\overline{G P Q}^{*}$ (iii) implies that $z(p, x) \in F(u)$ 
for the given $H$, as sought.

As the above arguments hold for any $H \in \mathcal{H}$ and any $u \in U_{N}$, the statement follows.

Proof of Theorem 4. Let $n=2$ and $\ell \geq 3$. Let Assumption 1 hold. Let $F \in \mathcal{F}$ be such that $F(u) \subseteq A \cap \mathbb{R}_{++}^{n \ell}$ for all $u \in U_{N}$. For each $i \in N$, the set of truthful messages is that defined in (1). First, we show that $F$ satisfies condition $\overline{P A 2}$. Suppose that $F$ is partially honest implementable by a natural price-allocation mechanism.

Take any $H \in \mathcal{H}$. Let us define the map $z: M \rightarrow Q^{2}$ as follows:

(a) if $F^{-1}(x, p) \neq \varnothing$ and $F^{-1}\left(x^{\prime}, p^{\prime}\right) \neq \varnothing$, then $z\left(p, x, p^{\prime}, x^{\prime}\right)=g\left(\left(p^{\prime}, x^{\prime}\right),(p, x)\right)$;

(b) if $F^{-1}(x, p) \neq \varnothing$ and $F^{-1}\left(x^{\prime}, p^{\prime}\right)=\varnothing$, with $x^{\prime} \in F(u)$ for some $u \in U_{N}$, then $z\left(p, x, p^{\prime}, x^{\prime}\right)=g\left(\left(p^{\prime}, x^{\prime}\right),(p, x)\right)$;

(c) if $F^{-1}(x, p)=\varnothing, F^{-1}\left(x^{\prime}, p^{\prime}\right) \neq \varnothing$, with $x \in F(u)$ for some $u \in U_{N}$, then $z\left(p, x, p^{\prime}, x^{\prime}\right)=$ $g\left(\left(p^{\prime}, x^{\prime}\right),(p, x)\right)$;

(d) otherwise, $z\left(p, x, p^{\prime}, x^{\prime}\right)=\mathbf{0}$.

Pick any $\left(p, x, p^{\prime}, x^{\prime}\right) \in \Delta \times A \times \Delta \times A$, with $F^{-1}(x, p) \neq \varnothing$ and $F^{-1}\left(x^{\prime}, p^{\prime}\right) \neq \varnothing$. Take any $u \in F^{-1}(x, p)$ and let $m_{i}=\left(p^{i}, x^{i}\right)=(p, x)$ for each $i \in N$. From forthrightness, it follows that $m \in N E\left(\gamma, \succcurlyeq^{u}\right)$ and $g(m)=x$. So, $g_{1}\left(M_{1},(p, x)\right) \subseteq L\left(x_{1}, u_{1}\right)$. Since this property holds for any $u \in F^{-1}(x, p)$, we have that $g_{1}\left(M_{1},(p, x)\right) \subseteq \Lambda_{1}^{F}(x, p)$. Similarly, it can be shown that $g_{2}\left(\left(p^{\prime}, x^{\prime}\right), M_{2}\right) \subseteq \Lambda_{2}^{F}\left(x^{\prime}, p^{\prime}\right) \equiv \cap_{u \in F^{-1}\left(x^{\prime}, p^{\prime}\right)} L\left(x_{2}^{\prime}, u_{2}\right)$. From the definition of the map $z$, it follows that $z\left(p, x, p^{\prime}, x^{\prime}\right)=g\left(\left(p^{\prime}, x^{\prime}\right),(p, x)\right)$. Then, $z\left(p, x, p^{\prime}, x^{\prime}\right) \in A$, with $z_{1}\left(p, x, p^{\prime}, x^{\prime}\right) \in \Lambda_{1}^{F}(x, p)$ and $z_{2}\left(p, x, p^{\prime}, x^{\prime}\right) \in \Lambda_{2}^{F}\left(x^{\prime}, p^{\prime}\right)$. Moreover, from the above arguments, $z\left(p, x, p^{\prime}, x^{\prime}\right)=g\left(\left(p^{\prime}, x^{\prime}\right),(p, x)\right)=x$ if $(p, x)=\left(p^{\prime}, x^{\prime}\right)$. This proves the assertion (a) of condition $\overline{P A 2}(\mathrm{i})$.

Pick any $\left(p, x, p^{\prime}, x^{\prime}\right) \in \Delta \times A \times \Delta \times A$ such that $F^{-1}(x, p) \neq \varnothing, F^{-1}\left(x^{\prime}, p^{\prime}\right)=\varnothing$ and $x^{\prime} \in F(u)$ for some $u \in U_{N}$. Then, from the definition of the map $z$, it follows $z\left(p, x, p^{\prime}, x^{\prime}\right) \in A$ and $z_{1}\left(p, x, p^{\prime}, x^{\prime}\right) \in \Lambda_{1}^{F}(x, p)$. This proves the assertion (b) of condition $\overline{P A 2}(\mathrm{i})$. Similarly, it can be shown that the map $z$ satisfies the assertion (c) of condition $\overline{P A 2}(\mathrm{i})$. Finally, the assertion (d) of condition $\overline{P A 2}$ is obvious by the definition of the mapping $z$. We conclude that condition $\overline{P A 2}(\mathrm{i})$ is verified.

Finally, we show that condition $\overline{P A 2}$ (ii) is satisfied by the given $F$ and $H \in \mathcal{H}$. Take any $u \in U_{N}$ and any $\left(p, x, p^{\prime}, x^{\prime}\right) \in \Delta \times A \times \Delta \times A$, with $(p, x) \neq\left(p^{\prime}, x^{\prime}\right)$. Suppose that the premises of condition $\overline{P A 2}$ (ii) are satisfied. Therefore, $z\left(p, x, p^{\prime}, x^{\prime}\right)=g\left(\left(p^{\prime}, x^{\prime}\right),(p, x)\right) \notin F(u)=$ $N A\left(\gamma, \succcurlyeq^{u}\right)$ for each $H^{\prime} \in \mathcal{H}$, and $\bar{m} \equiv\left(\left(p^{\prime}, x^{\prime}\right),(p, x)\right) \notin N E\left(\gamma, \succcurlyeq^{u}\right)$ for each $H^{\prime} \in \mathcal{H}$. It follows that $\left(\left(\hat{m}_{i}, \bar{m}_{l}\right), \bar{m}\right) \in \succ_{i}^{u}$ for some $i \in N$ and some $\hat{m}_{i} \in M_{i}$, with $i \neq l \in N$. This implies that $u_{i}\left(g_{i}\left(\hat{m}_{i}, \bar{m}_{l}\right)\right) \geq u_{i}\left(g_{i}(\bar{m})\right)$. However, since $g_{i}\left(\hat{m}_{i}, \bar{m}_{l}\right) \in g_{i}\left(M_{i}, \bar{m}_{l}\right) \subseteq$ $L\left(z_{i}\left(p, x, p^{\prime}, x^{\prime}\right), u_{i}\right)$, it follows that $u_{i}\left(g_{i}\left(\hat{m}_{i}, \bar{m}_{l}\right)\right)=u_{i}\left(g_{i}(\bar{m})\right)$. Therefore, the deviator $i$ is a partially honest agent, that is, $i \in H$. This means that $\hat{m}_{i} \equiv(\hat{p}, \hat{x}) \in T_{i}^{\gamma}(u, F)$ and $\bar{m}_{i} \notin T_{i}^{\gamma}(u, F)$, otherwise we fall into a contradiction.

Suppose that the deviator is $i \equiv 1$. Then, since $\bar{m}_{1} \notin T_{1}^{\gamma}(u, F), x^{\prime} \notin F(u)$ holds. Moreover, since $u_{1}\left(g_{1}\left(\hat{m}_{1}, \bar{m}_{2}\right)\right)=u_{1}\left(g_{1}(\bar{m})\right), g_{1}\left(\hat{m}_{1}, \bar{m}_{2}\right) \in \partial L\left(z_{1}\left(p, x, p^{\prime}, x^{\prime}\right), u_{1}\right)$ holds. Suppose that $F^{-1}(\hat{p}, \hat{x}) \neq \varnothing$. Then, $F^{-1}(\hat{p}, \hat{x}) \neq \varnothing$ and $F^{-1}(p, x) \neq \varnothing$. It follows from the definition of the mapping $z$ that $g_{1}\left(\hat{m}_{1}, \bar{m}_{2}\right)=z_{1}(p, x, \hat{p}, \hat{x})$. Otherwise, suppose that $F^{-1}(\hat{p}, \hat{x})=\varnothing$. Then, $F^{-1}(p, x) \neq \varnothing$ and $F^{-1}(\hat{p}, \hat{x})=\varnothing$, with $\hat{x} \in F(u)$. Again, by the definition of the mapping $z, g_{1}\left(\hat{m}_{1}, \bar{m}_{2}\right)=z_{1}(p, x, \hat{p}, \hat{x})$. In any case, we have found that $x^{\prime} \notin F(u)$ and $z_{1}(p, x, \hat{p}, \hat{x})=g_{1}\left(\hat{m}_{1}, \bar{m}_{2}\right) \in \partial L\left(z_{1}\left(p, x, p^{\prime}, x^{\prime}\right), u_{1}\right)$, with $\hat{x} \in F(u)$. Similar arguments can be applied to the case that the deviator is $i \equiv 2$. We conclude that $F$ satisfies condition $\overline{P A 2}($ ii), as sought. 
Next, we prove sufficiency. Suppose that $F$ satisfies condition $\overline{P A 2}$. We show that $F$ is partially honestly implemented by a natural price-allocation mechanism. Then, let the message space of each agent $i \in N$ be $M_{i} \equiv \Delta \times A$. Then, the message of the participant $i$, denoted $m_{i}=\left(p^{i}, x^{i}\right)$, consists of a price vector and a feasible allocation. Let $\gamma=(M, g)$ be a price-allocation mechanism.

Because $F$ satisfies condition $\overline{P A 2}$, there exists a map $z: M \rightarrow Q^{2}$ satisfying the requirements of condition $\overline{P A 2}(\mathrm{i})$. For any two different vertices $\bar{p}$ and $\overline{\bar{p}}$ of the unit simplex $\Delta, \Delta^{(\bar{p}, \bar{p})}$ denotes the 1-dimensional simplex with vertices $\bar{p}$ and $\overline{\bar{p}}$. Next, fix any two vertices of the simplex $\Delta$, that is, $\bar{p}$ and $\overline{\bar{p}}$. Let $\mathcal{B}_{\epsilon}(\bar{p} ; \overline{\bar{p}})$ be a closed ball around $\bar{p}$ with radius $\epsilon>0$ such that $\mathcal{B}_{\epsilon}(\bar{p} ; \bar{p})$ is a proper set of $\Delta^{(\bar{p}, \bar{p})}$ and $\overline{\bar{p}} \notin \mathcal{B}_{\epsilon}(\bar{p} ; \overline{\bar{p}})$. Let $\phi: \mathcal{B}_{\epsilon}(\bar{p} ; \overline{\bar{p}}) \rightarrow \Delta$ be a bijective function. This bijection always exists since $\mathcal{B}_{\epsilon}(\bar{p} ; \overline{\bar{p}})$ and $\Delta$ are cardinally equivalent. Let $\left(p^{2}, x^{2}\right)$ be any message reported by agent 2 such that $F^{-1}\left(p^{2}, x^{2}\right) \neq \varnothing$. Then, for each $(\hat{p}, \hat{x}) \in \mathcal{B}_{\epsilon}(\bar{p} ; \overline{\bar{p}}) \times A$, define $\tilde{z}\left(\hat{p}, \hat{x} ; p^{2}, x^{2}\right) \equiv$ $z\left(p^{2}, x^{2}, \phi(\hat{p}), \hat{x}\right)$, where $z\left(p^{2}, x^{2}, \phi(\hat{p}), \hat{x}\right)$ is allocation specified by the assertions (b) and (d) of condition $\overline{P A 2}(\mathrm{i})$. Similarly, for any $\left(p^{1}, x^{1}\right) \in \Delta \times A$ such that $F^{-1}\left(p^{1}, x^{1}\right) \neq \varnothing$, and for each $(\hat{p}, \hat{x}) \in \mathcal{B}_{\epsilon}(\bar{p} ; \overline{\bar{p}}) \times A$, define $\tilde{z}\left(\hat{p}, \hat{x} ; p^{1}, x^{1}\right) \equiv z\left(\phi(\hat{p}), \hat{x}, p^{1}, x^{1}\right)$, where $z\left(\phi(\hat{p}), \hat{x}, p^{1}, x^{1}\right)$ is the allocation specified specified by the assertions (c) and (d) of condition $\overline{P A 2}(\mathrm{i})$. Finally, for any $x \in \mathbb{R}_{+}^{n \ell}$ and $p \in \Delta, B\left(\Lambda_{i}^{F}(x, p)\right)$ is the upper boundary of $\Lambda_{i}^{F}(x, p)$, that is, $B\left(\Lambda_{i}^{F}(x, p)\right) \equiv\left\{y_{i} \in \mathbb{R}_{+}^{\ell} \mid y_{i} \in \Lambda_{i}^{F}(x, p)\right.$ and $\nexists z_{i} \in \Lambda_{i}^{F}(x, p)$ such that $\left.z_{i} \gg y_{i}\right\}$.

Define the outcome function $g$ of $\gamma$ as follows:

Rule 1: If $\left(p^{1}, x^{1}\right)=\left(p^{2}, x^{2}\right)=(p, x)$ and $F^{-1}(x, p) \neq \varnothing$, then $g(m)=x$.

Rule 2: If $\left(p^{1}, x^{1}\right) \neq\left(p^{2}, x^{2}\right)$ and $F^{-1}\left(x^{i}, p^{i}\right) \neq \varnothing$ for all $i \in N$, then $g(m)=z\left(p^{2}, x^{2}, p^{1}, x^{1}\right)$, where $z\left(p^{2}, x^{2}, p^{1}, x^{1}\right)$ is the allocation specified by the assertion (a) of condition $\overline{P A 2}(\mathrm{i})$.

Rule 3: If $\left(p^{1}, x^{1}\right) \neq\left(p^{2}, x^{2}\right), F^{-1}\left(x^{l}, p^{l}\right) \neq \varnothing$ for some $l \in N$ and $F^{-1}\left(x^{i}, p^{i}\right)=\varnothing$ for $i \in N \backslash\{l\}$, then:

Rule 3.1: If $p^{i} \in \Delta \backslash \mathcal{B}_{\epsilon}(\bar{p} ; \overline{\bar{p}})$, then $g(m)=x^{l}$;

Rule 3.2: If $p^{i} \in \mathcal{B}_{\epsilon}(\bar{p} ; \overline{\bar{p}})$, then $g_{l}(m)=\left(\Omega-g_{i}(m)\right)$ and

(a) If $i \equiv 1$, then

$$
g_{i}(m)=\left\{\begin{array}{ccc}
\tilde{z}_{i}\left(p^{i}, x^{i} ; p^{2}, x^{2}\right) & \text { if } & \tilde{z}_{i}\left(p^{i}, x^{i} ; p^{2}, x^{2}\right) \neq \mathbf{0} \\
x_{i}^{i} & \text { if } & \tilde{z}_{i}\left(p^{i}, x^{i} ; p^{2}, x^{2}\right)=\mathbf{0} \text { and } x_{i}^{i} \in \Lambda_{i}^{F}\left(x^{2}, p^{2}\right) \\
\hat{x}_{i} & & \text { otherwise }
\end{array}\right.
$$

(b) If $i \equiv 2$, then

$$
g_{i}(m)=\left\{\begin{array}{ccc}
\tilde{z}_{i}\left(p^{i}, x^{i} ; p^{1}, x^{1}\right) & \text { if } & \tilde{z}_{i}\left(p^{i}, x^{i} ; p^{1}, x^{1}\right) \neq \mathbf{0} \\
x_{i}^{i} & \text { if } & \tilde{z}_{i}\left(p^{i}, x^{i} ; p^{1}, x^{1}\right)=\mathbf{0} \text { and } x_{i}^{i} \in \Lambda_{i}^{F}\left(x^{1}, p^{1}\right) \\
\hat{x}_{i} & & \text { otherwise }
\end{array}\right.
$$

where $\left\{\hat{x}_{i}\right\} \equiv B\left(\Lambda_{i}^{F}\left(x^{l}, p^{l}\right)\right) \cap\left\{y_{i} \in \mathbb{R}_{+}^{\ell} \mid\right.$ there exists $\alpha \in \mathbb{R}_{+}$such that $\left.y_{i}=\alpha x_{i}\right\}$, where $l \in N \backslash\{i\}$

Rule 4: Otherwise, $g_{i^{*}}(m)=\Omega$ and $g_{j}(m)=0$ for all $j \neq i^{*}$, where $i^{*}$ is defined as follows. Without loss of generality, let us suppose that $\Omega_{1} \geq 1$. Let $\sum_{i \in N} x_{i 1}^{i}=t$. Furthermore, 
by Definition 3(ii), it follows that $x_{i 1}^{i} \in\left[0, \Omega_{1}\right]$ for all $i \in N$. Let $v$ be an integer such that $v \leq t<v+1$. Therefore, let $t=v+s$ where $s \in[0,1)$. It follows that there is a unique $i^{*} \in N$ such that $s \in\left[\frac{i^{*}-1}{n}, \frac{i^{*}}{n}\right)$.

According to the proposed construction, $\gamma$ is individually feasible and balanced; moreover, it satisfies forthrightness and the best response property.

We show that $F(u)=N A\left(\gamma, \succcurlyeq^{u}\right)$ for any $u \in U_{N}$ and any $H \in \mathcal{H}$. Then, take any $u \in U_{N}$ and any $H \in \mathcal{H}$.

First, we show that $F(u) \subseteq N A\left(\gamma, \succcurlyeq^{u}\right)$. To this end, let $x \in F(u)$ and $p \in \Pi(x, u)$. Let $m_{i}=\left(p^{i}, x^{i}\right)=(p, x) \in T_{i}^{\gamma}(u, F)$ for each $i \in N$. From Rule 1, it follows that $g(m)=x$. Suppose that agent $i \in N$ deviates from $m_{i}$ to a different message $m_{i}^{\prime} \in M_{i}$. By the definition of $g$, we have that any deviation of agent $i$ will get her to an outcome in $\Lambda_{i}^{F}(x, p)$, so that $g_{i}\left(M_{i}, m_{l}\right) \subseteq \Lambda_{i}^{F}(x, p)$, where $l \in N \backslash\{i\}$. Since $\Lambda_{i}^{F}(x, p) \subseteq L\left(x_{i}, u_{i}\right)$, these deviations are not profitable. As every agent is truthful, it follows that $x \in N A\left(\gamma, \succcurlyeq^{u}\right)$ for the given $H$.

Conversely, let $m \in N E\left(\gamma, \succcurlyeq^{u}\right)$ for this $H$. Since one cannot have a Nash equilibrium message profile under Rules 3-4, it follows that $m$ falls either into Rule 1 or into Rule 2 .

Suppose that $m$ falls into Rule 1 . Then, $m_{1}=(p, x)=m_{2}$, where $x \in F\left(u^{\prime}\right)$ and $p \in \Pi\left(x, u^{\prime}\right)$ for some $u^{\prime} \in U_{N}$. This rule implies that $g(m)=x$. Suppose that for a partially honest agent $h \in H, m_{h} \notin T_{h}^{\gamma}(u, F)$. Then, by changing $m_{h}$ into $m_{h}^{\prime}=\left(p^{h}, x^{h}\right) \in$ $T_{h}^{\gamma}(u, F)$, where $p^{h}=\overline{\bar{p}} \notin \mathcal{B}_{\epsilon}(\bar{p} ; \overline{\bar{p}})$, agent $h$ can induce Rule 3.1 as $F^{-1}\left(x^{h}, p^{h}\right)=\varnothing$ and $F^{-1}(x, p) \neq \varnothing$. Thus, $g\left(m_{h}^{\prime}, m_{l}\right)=x$, where $h \neq l \in N$. It follows that $\left(\left(m_{h}^{\prime}, m_{l}\right), m\right) \in \succ_{h}^{u}$, which contradicts that $m \in N E\left(\gamma, \succcurlyeq^{u}\right)$ for the given $H$. Therefore, $m_{h} \in T_{h}^{\gamma}(u, F)$ for all $h \in H$. We conclude that $x \in F(u)$ for this $H$, as sought.

Suppose that $m$ falls into Rule 2. Then, $g(m)=g\left(\left(p^{1}, x^{1}\right),\left(p^{2}, x^{2}\right)\right)=z\left(p^{2}, x^{2}, p^{1}, x^{1}\right)$. Notice that each agent $i \in N$ can induce Rule 3.2 and attain any bundle in $\partial \Lambda_{i}^{F}\left(x^{l}, p^{l}\right)$, where $l \in N \backslash\{i\}$. Thus, $\partial \Lambda_{i}^{F}\left(x^{l}, p^{l}\right) \subseteq g_{i}\left(M_{i}, m_{l}\right)$. Because $m \in N E\left(\gamma, \succcurlyeq^{u}\right)$, it follows that $\partial \Lambda_{i}^{F}\left(x^{l}, p^{l}\right) \subseteq L\left(z_{i}\left(p^{2}, x^{2}, p^{1}, x^{1}\right), u_{i}\right)$. Therefore, $\Lambda_{i}^{F}\left(x^{l}, p^{l}\right) \subseteq L\left(z_{i}\left(p^{2}, x^{2}, p^{1}, x^{1}\right), u_{i}\right)$ for each $i \in N$, given our supposition of agents' preferences. By the supposition that $m \in N E\left(\gamma, \succcurlyeq^{u}\right)$, it follows that for any $h \in H, g_{h}\left(m_{h}^{*}, m_{-h}\right) \notin \partial L\left(z_{h}\left(p^{2}, x^{2}, p^{1}, x^{1}\right), u_{h}\right)$ if $m_{h} \notin T_{h}^{\gamma}(u, F)$ and $m_{h}^{*} \in T_{h}^{\gamma}(u, F)$, otherwise we fall into a contradiction. Suppose that $m_{h} \in T_{h}^{\gamma}(u, F)$ for each $h \in H$. Then, condition $\overline{P A 2}\left(\right.$ ii) implies that $z\left(p^{2}, x^{2}, p^{1}, x^{1}\right) \in F(u)$ for the given $H$. Otherwise, let us suppose that $m_{h} \notin T_{h}^{\gamma}(u, F)$ for some $h \in H$. Define $H^{\prime} \equiv\left\{h \in H \mid m_{h} \notin T_{h}^{\gamma}(u, F)\right\}$. Therefore, $x^{h} \notin F(u)$ for all $h \in H^{\prime}$. Next, take any $h \in H^{\prime}$ and any $m_{h}^{\circ} \equiv\left(p^{\circ}, x^{\circ}\right) \in T_{h}^{\gamma}(u, F)$ such that $p^{\circ} \in \mathcal{B}_{\epsilon}(\bar{p} ; \overline{\bar{p}})$. Suppose that $h \equiv 1$. Then, $g_{h}\left(\left(p^{\circ}, x^{\circ}\right),\left(p^{2}, x^{2}\right)\right)=\tilde{z}_{h}\left(p^{\circ}, x^{\circ} ; p^{2}, x^{2}\right) \neq \mathbf{0}$ holds. Note that $\tilde{z}_{h}\left(\left(p^{\circ}, x^{\circ}\right) ;\left(p^{2}, x^{2}\right)\right) \neq$ 0 because $x^{\circ} \in F(u)$. Thus, $\tilde{z}_{h}\left(p^{\circ}, x^{\circ} ; p^{2}, x^{2}\right) \notin \partial L\left(z_{h}\left(p^{2}, x^{2}, p^{1}, x^{1}\right), u_{h}\right)$. By definition of $\tilde{z}\left(\cdot ; p^{2}, x^{2}\right), z_{h}\left(p^{2}, x^{2}, \phi\left(p^{\circ}\right), x^{\circ}\right) \notin \partial L\left(z_{h}\left(p^{2}, x^{2}, p^{1}, x^{1}\right), u_{h}\right)$. Since $\phi$ is a bijection and the previous arguments hold for any $p^{\circ} \in \mathcal{B}_{\epsilon}(\bar{p} ; \overline{\bar{p}})$ and any $x^{\circ} \in F(u), z_{h}\left(p^{2}, x^{2}, p, x\right) \notin$ $\partial L\left(z_{h}\left(p^{2}, x^{2}, p^{1}, x^{1}\right), u_{h}\right)$ for any $p \in \Delta$ and any $x \in F(u)$. Similar reasoning applies if $h \equiv 2$. Moreover, if $H \backslash H^{\prime}$ is not an empty set, then $m_{h} \in T_{h}^{\gamma}(u, F)$ for $h \in H \backslash H^{\prime}$. Condition $\overline{P A 2}$ (ii) implies that $z\left(p^{2}, x^{2}, p^{1}, x^{1}\right) \in F(u)$ for the given $H$, as sought.

As the above arguments hold for any $H \in \mathcal{H}$ and any $u \in U_{N}$, the statement follows.

Theorem 5. Let $n=2$ and $\ell=2$. Suppose that Assumption 1 holds. Let $F \in \mathcal{F}$ be such that $F(u) \subseteq A \cap \mathbb{R}_{++}^{n \ell}$ for all $u \in U_{N}$. Then, $F$ is partially honest implementable by a natural price-allocation mechanism in terms of DEFINITION 7 if and only if it satisfies condition $\overline{P A 2}$. 
Proof of Theorem 5. For each agent $i \in N$, the set of truthful messages is that defined in (1). It suffices to discuss the sufficiency part. Note that when $\ell=2$, then $\Delta$ is of dimension one, so that it is not possible to construct a simplex of dimension zero which contain two distinct vertices of $\Delta$. In contrast, since $\bar{\Delta}$ is a 2 -dimensional compact set, a 1-dimensional compact set $\bar{\Delta}^{(\bar{p}, \overline{\bar{p}})}$ which have the property that $F^{-1}(x, p)=\varnothing$ for any $x \in A \cap \mathbb{R}_{++}^{2 \times 2}$ and any $p \in \bar{\Delta}^{(\bar{p}, \overline{\bar{p}})}$ can be obtained from two different vertices $\bar{p}$ and $\overline{\bar{p}}$ of $\bar{\Delta}$. Then, fix $\bar{p} \equiv(0,0)$ and $\overline{\bar{p}} \equiv(0,1)$, and let $\bar{\Delta}(\bar{p}, \overline{\bar{p}})$ be the closed line connecting $\bar{p}$ and $\overline{\bar{p}}$. By the construction of $\bar{\Delta}(\bar{p}, \overline{\bar{p}})$ and the definition of the mapping $\varphi, \varphi(p) \in \Delta$ for any $p \in \bar{\Delta}^{(\bar{p}, \overline{\bar{p}})}$. Moreover, because $\bar{\Delta}(\bar{p}, \overline{\bar{p}})$ and $\bar{\Delta}$ are cardinally equivalent, there exists a bijective function $\bar{\phi}: \bar{\Delta}(\bar{p}, \overline{\bar{p}}) \rightarrow \bar{\Delta}$. Let $\left(p^{2}, x^{2}\right)$ be any message reported by agent 2 such that $F^{-1}\left(p^{2}, x^{2}\right) \neq \varnothing$. Then, for each $(\hat{p}, \hat{x}) \in$ $\bar{\Delta}^{(\bar{p}, \overline{\bar{p}})} \times A$, define $\bar{z}\left(\hat{p}, \hat{x} ; p^{2}, x^{2}\right) \equiv z\left(p^{2}, x^{2}, \varphi(\bar{\phi}(\hat{p})), \hat{x}\right)$, where $z\left(p^{2}, x^{2}, \varphi(\bar{\phi}(\hat{p})), \hat{x}\right)$ is allocation specified by the assertions (b) and (d) of $\overline{P A 2}(\mathrm{i})$. Similarly, for any $\left(p^{1}, x^{1}\right) \in$ $\Delta \times A$ such that $F^{-1}\left(p^{1}, x^{1}\right) \neq \varnothing$, and for each $(\hat{p}, \hat{x}) \in \bar{\Delta}^{(\bar{p}, \overline{\bar{p}})} \times A$, define $\bar{z}\left(\hat{p}, \hat{x} ; p^{1}, x^{1}\right) \equiv$ $z\left(\varphi(\bar{\phi}(\hat{p})), \hat{x}, p^{1}, x^{1}\right)$, where $z\left(\phi(\hat{p}), \hat{x}, p^{1}, x^{1}\right)$ is the allocation specified by the assertions (c) and (d) of $\overline{P A 2}(\mathrm{i})$. Based on these definitions, the desired result can be obtained by replacing, in Rule 3 of the mechanism constructed in the proof of Theorem $4, \mathcal{B}_{\epsilon}(\bar{p} ; \overline{\bar{p}})$ with $\bar{\Delta}^{(\bar{p}, \overline{\bar{p}})}$, the mapping $\tilde{z}\left(\cdot ; p^{2}, x^{2}\right)$ with $\bar{z}\left(\cdot ; p^{2}, x^{2}\right)$ (in Rule 3.2(a)), and the mapping $\tilde{z}\left(\cdot ; p^{1}, x^{1}\right)$ with $\bar{z}\left(\cdot ; p^{1}, x^{1}\right)$ (in Rule 3.2(b)).

Proof of Lemma 8. Let $n=2$. We show that the efficient and egalitarian-equivalent correspondence, $E E$, defined on $U_{N}=\bar{U}$, satisfies condition $P A 2$. To this end, take any $\left(p, x, p^{\prime}, x^{\prime}\right) \in \Delta \times A \times \Delta \times A$, with $(p, x) \neq\left(p^{\prime}, x^{\prime}\right), E E^{-1}(x, p) \neq \varnothing$ and $E E^{-1}\left(x^{\prime}, p^{\prime}\right) \neq \varnothing$. Then, for any $u \in E E^{-1}(x, p)$, there exists $\lambda_{(x, p)}^{u} \in(0,1)$ such that $u_{i}\left(x_{i}\right)=u_{i}\left(\lambda_{(x, p)}^{u} \Omega\right)$ for each $i \in N$. Let $\lambda_{(x, p)}^{\min }$ denote $\min _{u \in E E^{-1}(x, p)} \lambda_{(x, p)}^{u}$, so that $\lambda_{(x, p)}^{\min } \leq \lambda_{(x, p)}^{u}$ for any $u \in$ $E E^{-1}(x, p)$. Observe that $\lambda_{(x, p)}^{\min }$ always exists, given our suppositions. Moreover, given our suppositions, it cannot be that $p \cdot \lambda_{(x, p)}^{\min } \Omega \neq \max \left\{p \cdot x_{1}, p \cdot x_{2}\right\}$. To see it, assume, to the contrary, that $p \cdot \lambda_{(x, p)}^{\min } \Omega \neq \max \left\{p \cdot x_{1}, p \cdot x_{2}\right\}$. Without loss of generality, let $p \cdot x_{1}=$ $\max \left\{p \cdot x_{1}, p \cdot x_{2}\right\}$. Suppose that $p \cdot \lambda_{(x, p)}^{\min } \Omega<p \cdot x_{1}$. Then, there is no $u_{1} \in U_{1}$ such that $u_{1}\left(x_{1}\right)=u_{1}\left(\lambda_{(x, p)}^{\min } \Omega\right)$ and $p \in \delta u_{1}\left(x_{1}\right)$, which is a contradiction. Otherwise, suppose that $p \cdot \lambda_{(x, p)}^{\min } \Omega>p \cdot x_{1}$. Then, there exists $u \in E E^{-1}(x, p)$ such that $u_{i}\left(x_{i}\right)=u_{i}\left(\lambda_{(x, p)}^{\min } \Omega\right)$ and $p \in \delta u_{i}\left(x_{i}\right)$ for each $i \in N$. However, because $U_{N}=\bar{U}$ and $p \cdot \lambda_{(x, p)}^{\min } \Omega>p \cdot x_{1}$, there exists $u^{\prime} \in E E^{-1}(x, p)$, with $\lambda_{(x, p)}^{u^{\prime}}<\lambda_{(x, p)}^{\min }$, such that $u_{i}^{\prime}\left(x_{i}\right)=u_{i}^{\prime}\left(\lambda_{(x, p)}^{u^{\prime}} \Omega\right)$ and $p \in \delta u_{i}^{\prime}\left(x_{i}\right)$ for each $i \in N$, which produces a contradiction. Therefore, we conclude that $p \cdot \lambda_{(x, p)}^{\min } \Omega=$ $\max \left\{p \cdot x_{1}, p \cdot x_{2}\right\}$. In a similar way, it can be shown that there exists $\lambda_{\left(x^{\prime}, p^{\prime}\right)}^{\min } \in(0,1)$ such that $p^{\prime} \cdot \lambda_{\left(x^{\prime}, p^{\prime}\right)}^{\min } \Omega=\max \left\{p^{\prime} \cdot x_{1}^{\prime}, p^{\prime} \cdot x_{2}^{\prime}\right\}$. It follows that $p \cdot\left(1-\lambda_{(x, p)}^{\min }\right) \Omega=\min \left\{p \cdot x_{1}, p \cdot x_{2}\right\}$ and $p^{\prime} \cdot\left(1-\lambda_{\left(x^{\prime}, p^{\prime}\right)}^{\min }\right) \Omega=\min \left\{p^{\prime} \cdot x_{1}^{\prime}, p^{\prime} \cdot x_{2}^{\prime}\right\}$. Moreover, observe that $\lambda_{(x, p)}^{\min } \geq \frac{1}{2}$ and $\lambda_{\left(x^{\prime}, p^{\prime}\right)} \geq$ $\frac{1}{2}$.

From what we established above, it can easily be seen that $\lambda_{(x, p)}^{\min } \Omega \in \partial \Lambda_{1}^{E E}(x, p) \cap$ $\partial \Lambda_{2}^{E E}(x, p)$ and $\left(1-\lambda_{(x, p)}^{\min }\right) \Omega \in \Lambda_{1}^{E E}(x, p) \cap \Lambda_{2}^{E E}(x, p)$, because $\Lambda_{i}^{E E}(x, p) \supseteq H\left(p, x_{i}\right) \cap Q$ for each $i \in N^{22} \quad$ Likewise, $\lambda_{\left(x^{\prime}, p^{\prime}\right)}^{\min } \Omega \in \partial \Lambda_{1}^{E E}\left(x^{\prime}, p^{\prime}\right) \cap \partial \Lambda_{2}^{E E}\left(x^{\prime}, p^{\prime}\right)$ and $\left(1-\lambda_{\left(x^{\prime}, p^{\prime}\right)}^{\min }\right) \Omega \in$ $\Lambda_{1}^{E E}\left(x^{\prime}, p^{\prime}\right) \cap \Lambda_{2}^{E E}\left(x^{\prime}, p^{\prime}\right)$.

\footnotetext{
${ }^{22} H\left(p, x_{i}\right)$ is the hyperplane with normal $p$ through $x_{i}$.
} 
Now, let us first show that $E E$ satisfies condition $P A 2(i)$. To this end, it suffices to consider the following cases:

(1) $p \cdot x_{1}>p \cdot \frac{1}{2} \Omega>p \cdot x_{2}$ and $p^{\prime} \cdot x_{1}^{\prime} \geq p^{\prime} \cdot \frac{1}{2} \Omega \geq p^{\prime} \cdot x_{2}^{\prime}$;

(2) $p \cdot x_{1}>p \cdot \frac{1}{2} \Omega>p \cdot x_{2}$ and $p^{\prime} \cdot x_{2}^{\prime}>p^{\prime} \cdot \frac{1}{2} \Omega>p^{\prime} \cdot x_{1}^{\prime}$;

(3) $p \cdot x_{1} \geq p \cdot \frac{1}{2} \Omega \geq p \cdot x_{2}$ and $p^{\prime} \cdot x_{2}^{\prime}>p^{\prime} \cdot \frac{1}{2} \Omega>p^{\prime} \cdot x_{1}^{\prime}$;

(4) $p \cdot x_{1}>p \cdot \frac{1}{2} \Omega>p \cdot x_{2}$ and $p^{\prime} \cdot x_{2}^{\prime} \geq p^{\prime} \cdot \frac{1}{2} \Omega \geq p^{\prime} \cdot x_{1}^{\prime}$;

(5) $p \cdot x_{1}=p \cdot \frac{1}{2} \Omega=p \cdot x_{2}$ and $p^{\prime} \cdot x_{2}^{\prime}=p^{\prime} \cdot \frac{1}{2} \Omega=p^{\prime} \cdot x_{1}^{\prime}$.

For each of the above cases, we proceed according to whether the allocation specified in condition PA2(i) is (a) z $\left(p, x, p^{\prime}, x^{\prime}\right)$ or (b) $z\left(p^{\prime}, x^{\prime}, p, x\right)$.

$\diamond$ Case 1.a: $p \cdot x_{1}>p \cdot \frac{1}{2} \Omega>p \cdot x_{2}, p^{\prime} \cdot x_{1}^{\prime} \geq p^{\prime} \cdot \frac{1}{2} \Omega \geq p^{\prime} \cdot x_{2}^{\prime}$, and $z\left(p, x, p^{\prime}, x^{\prime}\right)$.

Since $p \cdot x_{1}>p \cdot x_{2}$, it follows that $\Lambda_{1}^{E E}(x, p)=H\left(p, x_{1}\right) \cap Q$. Let $z_{1}\left(p, x, p^{\prime}, x^{\prime}\right) \equiv$ $\left(1-\lambda_{\left(x^{\prime}, p^{\prime}\right)}^{\min }\right) \Omega$ and $z_{2}\left(p, x, p^{\prime}, x^{\prime}\right) \equiv \lambda_{\left(x^{\prime}, p^{\prime}\right)}^{\min } \Omega$. Then, $z_{2}\left(p, x, p^{\prime}, x^{\prime}\right) \in \Lambda_{2}^{E E}\left(x^{\prime}, p^{\prime}\right)$. Moreover, $\lambda_{\left(x^{\prime}, p^{\prime}\right)}^{\min } \geq \frac{1}{2}$ and $p \cdot x_{1}>p \cdot \frac{1}{2} \Omega$ imply that $z_{1}\left(p, x, p^{\prime}, x^{\prime}\right) \equiv\left(1-\lambda_{\left(x^{\prime}, p^{\prime}\right)}^{\min _{\Lambda}}\right) \Omega \in \stackrel{\circ}{H}\left(p, x_{1}\right) \cap Q \subseteq$ $\Lambda_{1}^{E E}(x, p)$. Therefore, under these configurations, EE meets condition PA2(i).

$\diamond$ Case 1.b: $p \cdot x_{1}>p \cdot \frac{1}{2} \Omega>p \cdot x_{2}, p^{\prime} \cdot x_{1}^{\prime} \geq p^{\prime} \cdot \frac{1}{2} \Omega \geq p^{\prime} \cdot x_{2}^{\prime}$, and $z\left(p^{\prime}, x^{\prime}, p, x\right)$.

Let $z_{1}\left(p^{\prime}, x^{\prime}, p, x\right) \equiv\left(1-\lambda_{(x, p)}^{\min }\right) \Omega$ and $z_{2}\left(p^{\prime}, x^{\prime}, p, x\right) \equiv \lambda_{(x, p)}^{\min } \Omega$. Since $\lambda_{(x, p)}^{\min }>\frac{1}{2}$ and $p^{\prime}$. $x_{1}^{\prime} \geq p^{\prime} \cdot \frac{1}{2} \Omega, z_{1}\left(p^{\prime}, x^{\prime}, p, x\right) \equiv\left(1-\lambda_{(x, p)}^{\min }\right) \Omega \in \stackrel{\circ}{H}\left(p^{\prime}, x_{1}^{\prime}\right) \cap Q \subseteq \Lambda_{1}^{E E}\left(x^{\prime}, p^{\prime}\right)$. Moreover, by definition, $z_{2}\left(p^{\prime}, x^{\prime}, p, x\right) \equiv \lambda_{(x, p)}^{\min } \Omega \in \Lambda_{2}^{E E}(x, p)$. Again, under these configurations, $E E$ satisfies condition $P A 2(\mathrm{i})$.

$\diamond$ Case 2.a: $p \cdot x_{1}>p \cdot \frac{1}{2} \Omega>p \cdot x_{2}, p^{\prime} \cdot x_{2}^{\prime}>p^{\prime} \cdot \frac{1}{2} \Omega>p^{\prime} \cdot x_{1}^{\prime}$, and $z\left(p, x, p^{\prime}, x^{\prime}\right)$.

Since $p \cdot x_{1}>p \cdot x_{2}$ and $p^{\prime} \cdot x_{2}^{\prime}>p^{\prime} \cdot x_{1}^{\prime}$, we have $\Lambda_{1}^{E E}(x, p)=H\left(p, x_{1}\right) \cap Q$ and $\Lambda_{2}^{E E}\left(x^{\prime}, p^{\prime}\right)=$ $H\left(p^{\prime}, x_{2}^{\prime}\right) \cap Q$. Let $z\left(p, x, p^{\prime}, x^{\prime}\right) \equiv\left(\frac{1}{2} \Omega, \frac{1}{2} \Omega\right)$. Then, $z\left(p, x, p^{\prime}, x^{\prime}\right) \in \stackrel{\circ}{\Lambda}_{1}^{E E}(x, p) \times \stackrel{\circ}{\Lambda}_{2}^{E E}\left(x^{\prime}, p^{\prime}\right)$. Since $z_{1}\left(p, x, p^{\prime}, x^{\prime}\right) \in \Lambda_{1}^{E E}(x, p)$ and $z_{2}\left(p, x, p^{\prime}, x^{\prime}\right) \in \Lambda_{2}^{E E}\left(x^{\prime}, p^{\prime}\right)$, it follows that $E E$ satisfies condition $P A 2(\mathrm{i})$ given our suppositions.

$\diamond$ Case 2.b: $p \cdot x_{1}>p \cdot \frac{1}{2} \Omega>p \cdot x_{2}, p^{\prime} \cdot x_{2}^{\prime}>p^{\prime} \cdot \frac{1}{2} \Omega>p^{\prime} \cdot x_{1}^{\prime}$, and $z\left(p^{\prime}, x^{\prime}, p, x\right)$.

We proceed according to whether $\lambda_{(x, p)}^{\min } \neq \lambda_{\left(x^{\prime}, p^{\prime}\right)}^{\min }$ or not. Firstly, let us consider the case that $\lambda_{(x, p)}^{\min } \neq \lambda_{\left(x^{\prime}, p^{\prime}\right)}^{\min }$. Then, let

$$
z\left(p^{\prime}, x^{\prime}, p, x\right) \equiv \begin{cases}\left(\left(1-\lambda_{(x, p)}^{\min }\right) \Omega, \lambda_{(x, p)}^{\min } \Omega\right) & \text { if } \lambda_{(x, p)}^{\min }>\lambda_{\left(x^{\prime}, p^{\prime}\right)}^{\min } \\ \left(\lambda_{\left(x^{\prime}, p^{\prime}\right)}^{\min } \Omega,\left(1-\lambda_{\left(x^{\prime}, p^{\prime}\right)}^{\min }\right) \Omega\right) & \text { if } \lambda_{(x, p)}^{\min }<\lambda_{\left(x^{\prime}, p^{\prime}\right)}^{\min }\end{cases}
$$

Then, if $\lambda_{(x, p)}^{\min }>\lambda_{\left(x^{\prime}, p^{\prime}\right)}^{\min }$, then $z_{1}\left(p^{\prime}, x^{\prime}, p, x\right) \in \stackrel{\circ}{\Lambda}_{1}^{E E}\left(x^{\prime}, p^{\prime}\right)$ and $z_{2}\left(p^{\prime}, x^{\prime}, p, x\right) \in \Lambda_{2}^{E E}(x, p)$; otherwise, $z_{1}\left(p^{\prime}, x^{\prime}, p, x\right) \in \Lambda_{1}^{E E}\left(x^{\prime}, p^{\prime}\right)$ and $z_{2}\left(p^{\prime}, x^{\prime}, p, x\right) \in \stackrel{\circ}{\Lambda}_{2}^{E E}(x, p)$. In either case, we have that condition $P A 2(\mathrm{i})$ is satisfied by $E E$.

Secondly, let us consider the case that $\lambda_{(x, p)}^{\min }=\lambda_{\left(x^{\prime}, p^{\prime}\right)}^{\min }$. For this case, we proceed according to whether there exists a suitable non-zero vector $\epsilon \in \mathbb{R}^{m} \backslash\{\mathbf{0}\}$ with $|\epsilon|>\mathbf{0}$ being sufficiently small, such that $\lambda_{(x, p)}^{\min } \Omega+\epsilon \in \Lambda_{1}^{E E}\left(x^{\prime}, p^{\prime}\right) \cap \Lambda_{2}^{E E}(x, p)$ or not.

Sub-case 2.b.1: $\lambda_{(x, p)}^{\min }=\lambda_{\left(x^{\prime}, p^{\prime}\right)}^{\min }$ and $\exists \epsilon \in \mathbb{R}^{m} \backslash\{\mathbf{0}\}$, with $|\epsilon|>\mathbf{0}: \lambda_{(x, p)}^{\min } \Omega+\epsilon \in \Lambda_{1}^{E E}\left(x^{\prime}, p^{\prime}\right) \cap$ $\Lambda_{2}^{E E}(x, p)$. 
In this case, by appropriately selecting such an $\epsilon$, we can find two feasible allocations $\left(\left(1-\lambda_{(x, p)}^{\min }\right) \Omega-\epsilon, \lambda_{(x, p)}^{\min } \Omega+\epsilon\right)$ and $\left(\lambda_{(x, p)}^{\min } \Omega+\epsilon,\left(1-\lambda_{(x, p)}^{\min }\right) \Omega-\epsilon\right)$ such that either $\left(1-\lambda_{(x, p)}^{\min }\right) \Omega-$ $\epsilon \in \stackrel{\circ}{\Lambda}_{1}^{E E}\left(x^{\prime}, p^{\prime}\right)$ or $\left(1-\lambda_{(x, p)}^{\min }\right) \Omega-\epsilon \in \stackrel{\circ}{\Lambda}_{2}^{E E}(x, p)$. Thus, if $\left(1-\lambda_{(x, p)}^{\min }\right) \Omega-\epsilon \in \stackrel{\circ}{\Lambda}_{1}^{E E}\left(x^{\prime}, p^{\prime}\right)$, let $z\left(p^{\prime}, x^{\prime}, p, x\right) \equiv\left(\left(1-\lambda_{(x, p)}^{\min }\right) \Omega-\epsilon, \lambda_{(x, p)}^{\min } \Omega+\epsilon\right)$, where $\lambda_{(x, p)}^{\min } \Omega+\epsilon \in \Lambda_{2}^{E E}(x, p)$. Otherwise, let $z\left(p^{\prime}, x^{\prime}, p, x\right) \equiv\left(\lambda_{(x, p)}^{\min } \Omega+\epsilon,\left(1-\lambda_{(x, p)}^{\min }\right) \Omega-\epsilon\right)$, where $\lambda_{(x, p)}^{\min } \Omega+\epsilon \in \Lambda_{1}^{E E}\left(x^{\prime}, p^{\prime}\right)$. This way of selecting $z\left(p^{\prime}, x^{\prime}, p, x\right)$ can be applied regardless of whether $p=p^{\prime}$ or not. In either case, we have that $z_{1}\left(p^{\prime}, x^{\prime}, p, x\right) \in \Lambda_{1}^{E E}\left(x^{\prime}, p^{\prime}\right)$ and $z_{2}\left(p^{\prime}, x^{\prime}, p, x\right) \in \Lambda_{2}^{E E}(x, p)$.

Sub-case 2.b.2: $\lambda_{(x, p)}^{\min }=\lambda_{\left(x^{\prime}, p^{\prime}\right)}^{\min }$ and $\nexists \epsilon \in \mathbb{R}^{m} \backslash\{\mathbf{0}\}$, with $|\epsilon|>\mathbf{0}: \lambda_{(x, p)}^{\min } \Omega+\epsilon \in \Lambda_{1}^{E E}\left(x^{\prime}, p^{\prime}\right) \cap$ $\Lambda_{2}^{E E}(x, p)$.

Then, take a non-zero vector $\epsilon \in \mathbb{R}^{m} \backslash\{\mathbf{0}\}$, with $|\epsilon|$ being sufficiently small, and consider $\lambda_{(x, p)}^{\min } \Omega+\epsilon$. Then, by appropriately selecting such an $\epsilon$, it is possible to have $\lambda_{(x, p)}^{\min } \Omega+\epsilon \epsilon$ $\Lambda_{1}^{E E}\left(x^{\prime}, p^{\prime}\right) \cup \Lambda_{2}^{E E}(x, p)$ and $\left(1-\lambda_{(x, p)}^{\min }\right) \Omega-\epsilon \in\left[\stackrel{\circ}{H}\left(p^{\prime}, x_{1}^{\prime}\right) \cap Q\right] \cup\left[\stackrel{\circ}{H}\left(p, x_{2}\right) \cap Q\right]$ such that $\lambda_{(x, p)}^{\min } \Omega+\epsilon \in \Lambda_{1}^{E E}\left(x^{\prime}, p^{\prime}\right)$ (resp., $\left.\lambda_{(x, p)}^{\min } \Omega+\epsilon \in \Lambda_{2}^{E E}(x, p)\right)$ implies that $\left(1-\lambda_{(x, p)}^{\min }\right) \Omega-\epsilon \epsilon$ $\left[\stackrel{\circ}{H}\left(p, x_{2}\right) \cap Q\right]$ (resp., $\left.\left(1-\lambda_{(x, p)}^{\min }\right) \Omega-\epsilon \in\left[\stackrel{\circ}{H}\left(p^{\prime}, x_{1}^{\prime}\right) \cap Q\right]\right)$. Therefore, if $\lambda_{(x, p)}^{\min } \Omega+\epsilon \epsilon$ $\Lambda_{2}^{E E}(x, p)$, let $z\left(p^{\prime}, x^{\prime}, p, x\right) \equiv\left(\left(1-\lambda_{(x, p)}^{\min }\right) \Omega-\epsilon, \lambda_{(x, p)}^{\min } \Omega+\epsilon\right)$, where the bundle $\left(\left(1-\lambda_{(x, p)}^{\min }\right) \Omega-\epsilon\right) \in$ $\stackrel{\circ}{\Lambda}_{1}^{E E}\left(x^{\prime}, p^{\prime}\right)$; otherwise, let $z\left(p^{\prime}, x^{\prime}, p, x\right) \equiv\left(\lambda_{(x, p)}^{\min } \Omega+\epsilon,\left(1-\lambda_{(x, p)}^{\min }\right) \Omega-\epsilon\right)$, where the bundle $\left(\left(1-\lambda_{(x, p)}^{\min }\right) \Omega-\epsilon\right) \in \stackrel{\circ}{\Lambda}_{2}^{E E}(x, p)$. This way of selecting $z\left(p^{\prime}, x^{\prime}, p, x\right)$ can be applied regardless of whether $p=p^{\prime}$ or not. In either case, under these configurations, $E E$ satisfies condition $P A 2(\mathrm{i})$.

$\diamond$ Cases 3-4.

Suitable punishment allocations can be found for Case 3 and Case 4 in a way similar to those shown for Case 2.a and Case 2.b.

$\diamond$ Case 5: $p \cdot x_{1}=p \cdot \frac{1}{2} \Omega=p \cdot x_{2}$ and $p^{\prime} \cdot x_{2}^{\prime}=p^{\prime} \cdot \frac{1}{2} \Omega=p^{\prime} \cdot x_{1}^{\prime}$.

We consider only the sub-case that the punishment allocation is $z\left(p, x, p^{\prime}, x^{\prime}\right)$, since the other sub-case can be proved in a similar way. Then, $\Lambda_{1}^{E E}(x, p)=H\left(p, x_{1}\right) \cap Q$ and $\Lambda_{2}^{E E}\left(x^{\prime}, p^{\prime}\right)=$ $H\left(p^{\prime}, x_{2}^{\prime}\right) \cap Q$. If $p \neq p^{\prime}$, then we can easily find a feasible allocation $z \in A$ such that $z_{1} \in \stackrel{\circ}{H}\left(p, x_{1}\right) \cap Q$ and $z_{2} \in \stackrel{\circ}{H}\left(p^{\prime}, x_{2}^{\prime}\right) \cap Q$. Therefore, let $z\left(p, x, p^{\prime}, x^{\prime}\right) \equiv z$ whenever $p \neq p^{\prime}$. If $p=p^{\prime}$, then $z\left(p, x, p^{\prime}, x^{\prime}\right) \equiv\left(\frac{1}{2} \Omega, \frac{1}{2} \Omega\right)$, so that $z_{1}\left(p, x, p^{\prime}, x^{\prime}\right) \in \partial \Lambda_{1}^{E E}(x, p)$ and $z_{2}\left(p, x, p^{\prime}, x^{\prime}\right) \in \partial \Lambda_{2}^{E E}\left(x^{\prime}, p^{\prime}\right)$. In either case, we have that condition $P A 2(\mathrm{i})$ is satisfied by EE.

Since the above arguments hold for any $\left(p, x, p^{\prime}, x^{\prime}\right) \in \Delta \times A \times \Delta \times A$, with $(p, x) \neq\left(p^{\prime}, x^{\prime}\right)$, $E E^{-1}(x, p) \neq \varnothing$ and $E E^{-1}\left(x^{\prime}, p^{\prime}\right) \neq \varnothing$, it follows that $E E$, defined on $U_{N}=\bar{U}$, satisfies condition $P A 2(\mathrm{i})$, as sought.

Finally, let us show that $E E$ satisfies condition $P A 2($ ii). By the above constructions of $z\left(p, x, p^{\prime}, x^{\prime}\right)$ and $z\left(p^{\prime}, x^{\prime}, p, x\right)$, the only case in which condition $P A 2(i i)$ is not vacuously satisfied is that of Case 5, when $p=p^{\prime}$. In what follows, we show this case by considering only the sub-case that the punishment allocation is $z\left(p, x, p^{\prime}, x^{\prime}\right)$, because the other sub-case can be proved in a similar way. Then, let $z\left(p, x, p^{\prime}, x^{\prime}\right) \equiv\left(\frac{1}{2} \Omega, \frac{1}{2} \Omega\right)$, with 
$z_{1}\left(p, x, p^{\prime}, x^{\prime}\right) \in \Lambda_{1}^{E E}(x, p)=H\left(p, x_{1}\right) \cap Q$ and $z_{2}\left(p, x, p^{\prime}, x^{\prime}\right) \in \Lambda_{2}^{E E}\left(x^{\prime}, p^{\prime}\right)=H\left(p^{\prime}, x_{2}^{\prime}\right) \cap Q$. Moreover, suppose that, for some $u^{*} \in U_{N}$, we have that $\Lambda_{1}^{E E}(x, p) \subseteq L\left(z_{1}\left(p, x, p^{\prime}, x^{\prime}\right), u_{1}^{*}\right)$ and $\Lambda_{2}^{E E}\left(x^{\prime}, p^{\prime}\right) \subseteq L\left(z_{2}\left(p, x, p^{\prime}, x^{\prime}\right), u_{2}^{*}\right)$. Then, $z\left(p, x, p^{\prime}, x^{\prime}\right) \in P\left(u^{*}\right)$. Furthermore, because $z\left(p, x, p^{\prime}, x^{\prime}\right) \equiv\left(\frac{1}{2} \Omega, \frac{1}{2} \Omega\right)$, it follows that $z\left(p, x, p^{\prime}, x^{\prime}\right) \in E E\left(u^{*}\right)$, as sought. We conclude that condition $P A 2(\mathrm{ii})$ is satisfied by $E E$. This completes the proof.

\section{References}

[1] Bowles, S., Gintis, H., 2000. Walrasian Economics in Retrospect. Quarterly Journal of Economics, 115, 1411-1439.

[2] Cabrales, A., Serrano, R., 2011. Implementation in adaptive better-response dynamics: Towards a general theory of bounded rationality in mechanisms. Games and Economic Behavior, 73, 360-374.

[3] Camerer, C.F., 2003. Behavioral Game Theory: Experiments in Strategic Interaction. Princeton University Press, Princeton, New Jersey, USA.

[4] Corchón, L., Herrero, C., 2004. A decent proposal. Spanish Economic Review, 6, 107125.

[5] Dutta, B., Sen, A., 2012. Nash implementation with partially honest individuals. Games and Economic Behavior, 74, 154-169.

[6] Dutta, B., Sen, A., Vohra, R., 1995. Nash implementation through elementary mechanisms in economic environments. Economic Design, 1, 173-204.

[7] Eliaz, K., 2002. Fault-Tolerant Implementation. Review of Economic Studies, 69, 589610.

[8] Elster, J., 1989. Social Norms and Economic Theory. Journal of Economic Perspectives, 3, 99-117.

[9] Elster, J., 1998. Emotions and Economic Theory. Journal of Economic Literature, 36, 47-74.

[10] Glazer, J., Rubinstein, A., 1998. Motives and Implementation: On the Design of Mechanisms to Elicit Opinions. Journal of Economic Theory, 79, 157-173.

[11] Greene, J. D., Paxton, J.M., 2009. Patterns of Neural Activity Associated with Honest and Dishonest Moral Decisions. Proceedings of National Academy of Science, 106, 1250612511.

[12] Hurkens, S., Kartik, N., 2009. Would I Lie to You? On Social Preferences and Lying Aversion. Experimental Economics, 12, 180-192.

[13] Hurwicz, L., 1960. Optimality and Informational Efficiency in Resource Allocation Processes. In Arrow, K. J., Karlin, S., and Suppes, P. (eds.), Mathematical Methods in the Social Sciences. Stanford University Press, USA, 27-46. 
[14] Hurwicz, L., 1986a. On the Implementation of Social Choice Rules in Irrational Societies. In: Heller, W., Starr, R., Starrett, D. (eds.), Social Choice and Public Decision Making, Essays in Honor of Kenneth J. Arrow. Cambridge University Press, USA, Volume I, 75-96.

[15] Hurwicz, L., 1986b. On informational decentralization and efficiency in resource allocation mechanism. In: Reider, S., (ed.), Studies in Mathematical Economics. Mathematical Association of America, Washington D.C., USA, 238-350.

[16] Jackson, M., 2001. A crash course in implementation theory. Social Choice and Welfare, 18, 655-708.

[17] Kartik, N., Tercieux, O., 2011. Implementation with evidence. Accepted for publication at Theoretical Economics.

[18] Kreps, D.M., 1997. Intrinsic Motivation and Extrinsic Incentives. American Economic Review, 87, 359-364.

[19] Lombardi, M., Yoshihara, N., 2011a. Partially-honest Nash implementation: Characterization results. Mimeo.

[20] Lombardi, M., Yoshihara, N., 2011b. A Full Characterization of Nash Implementation with Strategy Space Reduction. Mimeo.

[21] Lombardi, M., Yoshihara, N., 2011c. Natural implementation with partially honest agents by quantity mechanisms. Mimeo.

[22] Lombardi, M., Yoshihara, N., 2011d. Partially honest Nash implementation by selfrelevant mechanisms. Mimeo.

[23] Maskin, E., 1999. Nash equilibrium and welfare optimality. Review of Economic Studies, $66,23-38$.

[24] Maskin, E., Sjöström, T., 2002. The theory of implementation. In: Arrow, K., Sen, A.K., Suzumura, K. (eds.), Handbook of Social Choice and Welfare. Amsterdam: Elsevier Science, Volume I, 238-288.

[25] Saijo, T., 1988. Strategy space reduction in Maskin's theorem: sufficient conditions for Nash implementation. Econometrica, 56, 693-700.

[26] Saijo, T., Tatamitani, Y., Yamato, T., 1996a. Toward natural implementation. International Economic Review, 37, 949-980.

[27] Saijo, T., Tatamitani, Y., Yamato, T., 1996b. Natural implementation with a simple punishment. The Japanese Economic Review, 47, 170-185.

[28] Saijo, T., Tatamitani, Y., Yamato, T., 1999. Characterizing natural implementability: The fair and Walrasian correspondences. Games and Economic Behavior, 28, 271-293.

[29] Sen, A.K., 1997. Maximization and the act of choice. Econometrica, 65, 745-779.

[30] Sjöström, T., 1996. Implementation by demand mechanisms. Economic Design, 1, 343354. 
[31] Suzumura, K., Xu, Y., 2001. Characterizations of Consequentialism and Nonconsequentialism. Journal of Economic Theory, 101, 423-436.

[32] Tatamitani, Y., 2001. Implementation by self-relevant mechanisms. Journal of Mathematical Economics, 35, 427-444.

[33] Thomson, W., 2005. Divide and permute. Games and Economic Behavior, 52, 186-200. 